\title{
Development
}

\section{Auditory Brainstem Deficits from Early Treatment with a CSF1R Inhibitor Largely Recover with Microglial Repopulation}

\author{
(Diedre Milinkeviciute, Sima M. Chokr, and ${ }^{\circledR}$ Karina S. Cramer
}

https://doi.org/10.1523/ENEURO.0318-20.2021

Department of Neurobiology and Behavior, University of California, Irvine, CA 92697

\begin{abstract}
Signaling between neurons and glia is necessary for the formation of functional neural circuits. A role for microglia in the maturation of connections in the medial nucleus of the trapezoid body (MNTB) was previously demonstrated by postnatal microglial elimination using a colony stimulating factor 1 receptor (CSF1R). Defective pruning of calyces of Held and significant reduction of the mature astrocyte marker glial fibrillary acidic protein (GFAP) were observed after hearing onset. Here, we investigated the time course required for microglia to populate the mouse MNTB after cessation of CSF1R inhibitor treatment. We then examined whether defects seen after microglial depletion were rectified by microglial repopulation. We found that microglia returned to control levels at four weeks of age ( $18 \mathrm{~d}$ postcessation of treatment). Calyceal innervation of MNTB neurons was comparable to control levels at four weeks and GFAP expression recovered by seven weeks. We further investigated the effects of microglia elimination and repopulation on auditory function using auditory brainstem recordings (ABRs). Temporary microglial depletion significantly elevated auditory thresholds in response to 4. 8, and $12 \mathrm{kHz}$ at four weeks. Treatment significantly affected latencies, interpeak latencies, and amplitudes of all the ABR peaks in response to many of the frequencies tested. These effects largely recovered by seven weeks. These findings highlight the functions of microglia in the formation of auditory neural circuits early in development. Further, the results suggest that microglia retain their developmental functions beyond the period of circuit refinement.
\end{abstract}

Key words: auditory brainstem; calyx of Held; depletion; GFAP; microglia; MNTB

\section{Significance Statement}

Auditory brainstem pathways are optimized for their special functions that are shaped during development, which relies on the functions of non-neuronal cells, such as microglia and astrocytes. When microglia were pharmacologically eliminated during the early postnatal period with a colony stimulating factor 1 receptor (CSF1R) inhibitor, excess calyces were not pruned and astrocytes did not mature properly in the auditory brainstem. Here, we show that once this drug is withdrawn, microglia gradually return to the auditory nuclei. After microglia reemerge in the medial nucleus of the trapezoid body (MNTB), synaptic pruning of calyces of Held resumes and maturation of astrocytes and auditory function recover. The findings suggest that the auditory brainstem pathways can be shaped by microglia even after their normal period of circuit development.

Received July 16, 2020; accepted January 13, 2021; First published February 8, 2021.

The authors declare no competing financial interests.
Author contributions: G.M., S.M.C., and K.S.C. designed research; G.M. and S.M.C. performed research; G.M., S.M.C., and K.S.C. analyzed data; G.M., S.M.C., and K.S.C. wrote the paper. 


\section{Introduction}

Specialized neural circuits in the auditory brainstem carry out computations needed for auditory processing, including sound source localization (Grothe et al., 2010). Auditory nerve fibers synapse onto bushy cells in the anteroventral cochlear nucleus (AVCN; Held, 1893; Brawer and Morest, 1975; Ryugo and Sento, 1991; Lauer et al., 2013). Globular bushy cell (GBC) axon terminals form the elaborate calyx of Held (Held, 1893), which contacts principal neurons in the contralateral medial nucleus of the trapezoid body (MNTB; Morest, 1968; Smith et al., 1991; Kandler and Friauf, 1993). MNTB neurons inhibit neurons in the lateral superior olive (LSO; Kuwabara and Zook, 1991; Tollin, 2003). LSO neurons also receive tonotopically matched excitatory input from the ipsilateral side and use the balance between inhibition and excitation to compute interaural intensity differences (Boudreau and Tsuchitani, 1968; Sanes and Rubel, 1988; Grothe, 2003). This pathway, which allows for fast and reliable transmission of sound signals (Borst and Sakmann, 1996; Trussell, 1999; Taschenberger et al., 2002), relies on precision in developmental mechanisms.

Microglia mediate major functions in neural development (Reemst et al., 2016; Nelson and Lenz, 2017) and formation of neuronal circuits (Miyamoto et al., 2016; Basilico et al., 2019) through their roles in synapse elimination (Schafer and Stevens, 2010; Paolicelli et al., 2011; Schafer et al., 2012; Neniskyte and Gross, 2017), construction of new synapses (Parkhurst et al., 2013; Miyamoto et al., 2016), and synaptic remodeling (Tremblay et al., 2010; Schafer et al., 2012; Weinhard et al., 2018). When microglial function is disturbed, excess synapses persist in adulthood, resulting in anatomic abnormalities in neural circuits (Kettenmann et al., 2013).

Microglia are sparse in MNTB during the first postnatal week, then peak in density just after hearing onset at two weeks (Dinh et al., 2014). Microglia are found in close proximity to the developing calyx of Held (Holcomb et al., 2013; Dinh et al., 2014). From multiple small "protocalyces" contacting a single MNTB neuron (Kandler and Friauf, 1993), a single dominant calyx of Held emerges and monoinnervation of MNTB neurons is established (Morest, 1968; Sätzler et al., 2002; Hoffpauir et al., 2006; Holcomb et al., 2013). Microglial elimination before hearing onset results in

This work was supported by National Institutes of Health Grants R01 DC010796, R01NS083801 (National Institute of Neurological Disorders and Stroke), R01AG056768 (National Institute on Aging). This study was made possible in part through access to the Optical Biology Core Facility of the Developmental Biology Center, a shared resource supported by the Cancer Center Support Grant CA-62203 and Center for Complex Biological Systems Support Grant GM-076516 at the University of California, Irvine.

Acknowledgements: We thank Dr. Adeela Syed at the Optical Biology Core Facility of the Developmental Biology Center at the University of California, Irvine, for her advice and help with confocal imaging and analysis.

Correspondence should be addressed to Karina S. Cramer at cramerk@ uci.edu.

https://doi.org/10.1523/ENEURO.0318-20.2021

Copyright @ 2021 Milinkeviciute et al.

This is an open-access article distributed under the terms of the Creative Commons Attribution 4.0 International license, which permits unrestricted use, distribution and reproduction in any medium provided that the original work is properly attributed. excess polyinnervated neurons, signifying a microglial role in the development of neural circuits in the brainstem (Milinkeviciute et al., 2019).

Like microglia, astrocytes support synaptic development and remodeling. Despite differences between microglia and astrocytes in terms of their origin and structure, these cell types exhibit coordinated responses to insult (Schiweck et al., 2018; Vainchtein and Molofsky, 2020). Astrocytes and microglia communicate through bidirectional signaling and shared responses to environmental signals (Jha et al., 2019; Vainchtein and Molofsky, 2020). Microglia can influence expression of astrocytic proteins (Elmore et al., 2014; Spangenberg et al., 2016; Jin et al., 2017) and astrocytes can signal to microglia through several pathways (Bialas and Stevens, 2013; Vainchtein et al., 2018). In the auditory brainstem, microglial depletion prevented the emergence of astrocytic expression of glial fibrillary acidic protein (GFAP), a marker for mature astrocytes (Wofchuk and Rodnight, 1995; Gomes et al., 1999; Middeldorp and Hol, 2011), further highlighting the relationship between the two glial populations (Milinkeviciute et al., 2019).

Here, we tested whether microglia can return after postnatal pharmacological elimination, and whether microglial repopulation can restore development of auditory brainstem pathways. We further tested whether temporary postnatal microglial elimination has an effect on auditory function. We depleted microglia during the early postnatal period with an inhibitor of colony stimulating factor 1 receptor (CSF1R; Milinkeviciute et al., 2019), which is essential for microglial survival and proliferation (Stanley et al., 1997; Erblich et al., 2011). We then allowed microglia to repopulate by cessation of treatment after postnatal day $(P) 10$. We found that microglia slowly recolonize the brainstem, first in lateral regions and later in progressively more medial regions. We found that defects present at P13 (Milinkeviciute et al., 2019), synaptic pruning and decreased expression of GFAP, were both corrected when microglia repopulated the MNTB. Additionally, we evaluated the overall auditory brainstem function of these animals using ABRs. We found elevated auditory thresholds, increased latencies and interpeak latencies, and decreased amplitudes in almost all ABR peaks in response to most of the frequencies tested at four weeks of age. ABRs were partially restored to normal by seven weeks of age. These results suggest that microglia have early developmental roles in synaptic pruning of calyces of Held, promoting astrocyte maturation, and maturation of auditory brainstem function. Our findings further suggest that microglia can act at later developmental times, at least in part, to restore normal formation of auditory brainstem circuits.

\section{Materials and Methods}

\section{Animals and treatment}

We used eight P14, seven P18, 18 three-week-old, 37 four-week-old, and 26 seven-week-old wild-type C57BL/ 6 mice of both sexes. All animal procedures were performed in accordance with the University of California, 
Irvine animal care committee's regulations. Mice were housed in groups, with a maximum of five adult mice per cage. Mice were reared in a standard dark/light cycle, fed a standard diet and received food and water ad libitum. Whenever possible, mice that were used for ABRs were also used for histology with exception of pruning analysis. Each litter was divided into control and experimental mice. Experimental mice were repeatedly injected with BLZ945 (subcutaneous injections at P2, P4, P6, P8, and P10; MW: 398.48, MedChem Express HY-12768/CS3971), a small molecule inhibitor of CSF1R (Pyonteck et al., 2013), to eliminate microglia as previously described (Milinkeviciute et al., 2019). BLZ945 was dissolved in dimethylsulfoxide (DMSO; D136-1; Fisher Scientific) and $0.01 \mathrm{ml}$ of solution $(200 \mathrm{mg} / \mathrm{kg}$ ) was administered subcutaneously every $2 \mathrm{~d}$ starting from P2 with the last injection delivered at P10. Control mice were injected with DMSO following the same protocol. After cessation of treatment, mice were killed when they were 14 or $18 \mathrm{~d}$ or three, four, or seven weeks old (Fig. 1A).

\section{Tissue preparation and immunofluorescence}

Mice were weighed and perfused transcardially with $0.9 \%$ saline followed by $4 \%$ paraformaldehyde (PFA) in $0.1 \mathrm{M}$ phosphate buffer, pH 7.3 (PBS) at three, four, or seven weeks (Fig. 1A). Brainstems were dissected and postfixed overnight in PFA solution. Brains were then transferred to a $30 \%$ sucrose solution in $0.1 \mathrm{~m}$ PBS overnight, embedded in OCT mounting medium, and sectioned coronally at $18 \mu \mathrm{m}$ using a cryostat (CM 1850-3-1; Leica Microsystems). Tissue was mounted on chromealum-coated glass slides in a one-in-five series. Mounted sections were surrounded with a PAP pen hydrophobic barrier and rinsed in $0.1 \mathrm{M}$ PBS for $10 \mathrm{~min}$. Tissue was incubated for $5 \mathrm{~min}$ in $0.1 \%$ sodium dodecyl sulfate in $0.1 \mathrm{M}$ PBS solution for antigen retrieval followed by $310 \mathrm{~min}$ washes in $0.1 \mathrm{M}$ PBS. Sections were then blocked with normal goat blocking solution containing 5\% normal goat serum (NGS; Vector Laboratories S-1000) and $0.3 \%$ Triton X-100 (Acros 9002-93-1) in 0.1 M PBS in a humidity chamber at room temperature. After $1 \mathrm{~h}$, primary antibodies were applied (Table 1). The next day, the tissue was rinsed in $0.1 \mathrm{~m}$ PBS and incubated for $1 \mathrm{~h}$ in goat anti-rabbit or anti-chicken secondary antibody tagged with an Alexa (Invitrogen) fluorophore (Table 1). Sections were washed in $0.1 \mathrm{~m}$ PBS and incubated in blue or red fluorescent Nissl stain (NeuroTrace $435 / 455$ or $530 / 615$, Life Technologies N21482 or N21479) diluted in 1:200 in $0.3 \%$ Triton X-100 in $0.1 \mathrm{M}$ PBS for $1 \mathrm{~h}$. Tissue was then rinsed and coverslipped with Glycergel mounting medium (Dako C0563).

\section{Areal coverage analysis}

We acquired $20 \times$ magnification images of sections throughout the rostro-caudal extent of the MNTB on both sides of the midline using a Zeiss Axioskop-2 microscope, an Axiocam camera, and Axiovision software. For each subject, a series of $20 \times$ multichannel fluorescent photographs spanning the rostro-caudal extent of the MNTB was taken and collected as an image stack in FIJI (Schindelin et al., 2012). The Nissl channel was used to outline the MNTB [a region of interest (ROI)] in every section in FIJI. A stack of ROls from each animal was used to analyze the areal coverage of IBA1 or GFAP immunolabeling in the corresponding MNTB region as described in Milinkeviciute et al. (2019).

\section{Neuronal tracing and confocal analysis}

We used four control and four BLZ-treated mice and five control and five BLZ945-treated mice at three and four weeks, respectively, for neuronal tracing. After transcardial perfusion with artificial CSF (aCSF; $130 \mathrm{~mm} \mathrm{NaCl,}$ $3 \mathrm{~mm} \mathrm{KCl}, 1.2 \mathrm{~mm} \mathrm{KH}_{2} \mathrm{PO}_{4}, 20 \mathrm{~mm} \mathrm{NaHCO}_{3}, 3$ mм HEPES, $10 \mathrm{~mm}$ glucose, $2 \mathrm{~mm} \mathrm{CaCl}_{2}$, and $1.3 \mathrm{~mm} \mathrm{MgSO}_{4}$ perfused with $95 \% \mathrm{O}_{2}$ and $5 \% \mathrm{CO}_{2}$ ), brains were quickly dissected and placed in a chamber with oxygenated aCSF. We temporarily placed the brain in a Petri dish with aCSF and filled calyces of Held in the MNTB with a rhodamine dextran amine (RDA; MW 3000, Invitrogen) solution $(6.35 \%$ RDA with $0.4 \%$ Triton X-100 in PBS). Pulses of RDA were delivered through a pulled glass micropipette in the ventral acoustic stria (VAS) close to the midline using an Electro Square Porator (ECM830; BTX) at a rate of five pulses per second (pps) at $55 \mathrm{~V}$ for $50 \mathrm{~ms}$. As a result, axonal projections from the AVCN terminating in calyces of Held in the MNTB were sparsely labeled with RDA on both sides of the brainstem. The brain was then transferred back into the aCSF chamber for $\sim 2 \mathrm{~h}$ under continuous oxygenation to allow dye transport along the GBC axons. The brain was then placed in 4\% PFA overnight then transferred to $30 \%$ sucrose in $0.1 \mathrm{~m}$ PBS and later cryosectioned in the coronal plane at $18 \mu \mathrm{m}$. Sections were mounted on chrome-alum-coated glass slides in a one-in-five series and were immunolabeled with antiVGLUT1/2 antibody (Table 1) following the protocol described above.

Stained slides were analyzed using confocal microscopy (Leica SP8, 63× oil objective, zoom: 1.5, pinhole: 1). NissI, RDA, and VGLUT1/2 z-stack images of calyces of Held were acquired at a resolution of $1024 \times 1024$ pixels, with a z-step size of $0.5 \mu \mathrm{m}$ (Grande et al., 2014; Wang et al., 2018; Milinkeviciute et al., 2019). Gain and offset were set for each fluorescent channel and each slide separately and adjusted if labeling intensity was noticeably different between sections on the same slide. Calyces throughout the MNTB were sparsely and randomly labeled with RDA, thus, the entire mediolateral extent of the MNTB was randomly sampled. If the number of labeled calyces from one slide was too small $(<3)$, an additional slide was used (Table 2).

Image stacks were analyzed using the surface module in Imaris software (v9.5.1; Bitplane). Surfaces of RDAfilled calyces that were complete or near complete and that had adequate VGLUT1/2 immunofluorescence as well as a visible preterminal axon segment were reconstructed using a 0.4 surface detail and 0.4 background subtraction settings. These settings were determined before the analysis and were chosen so as to most reliably reconstruct the surface of the calyx of Held. If necessary, 


\section{eNeuro}

A
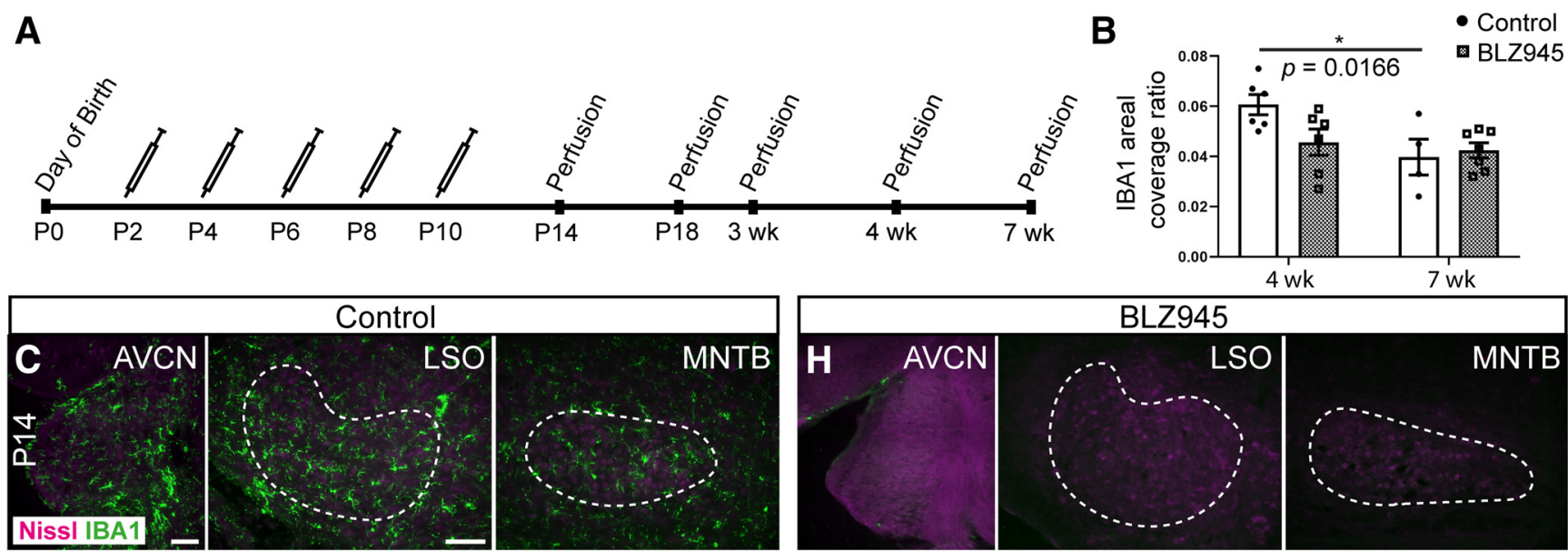
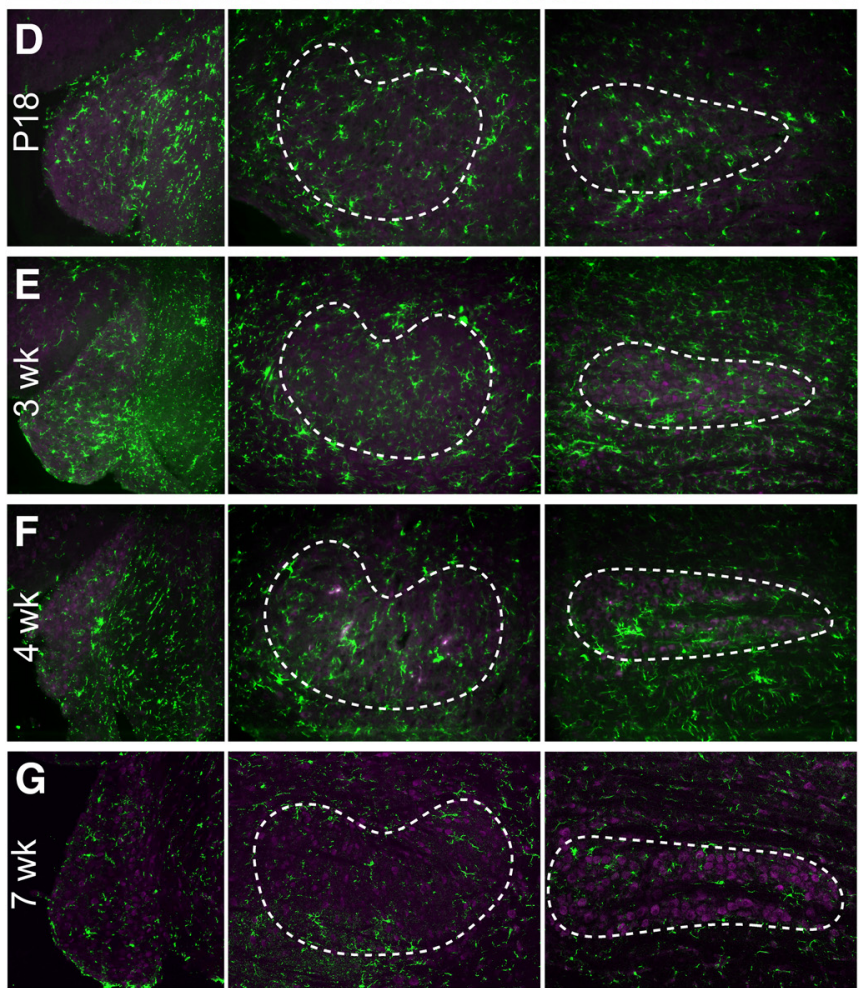
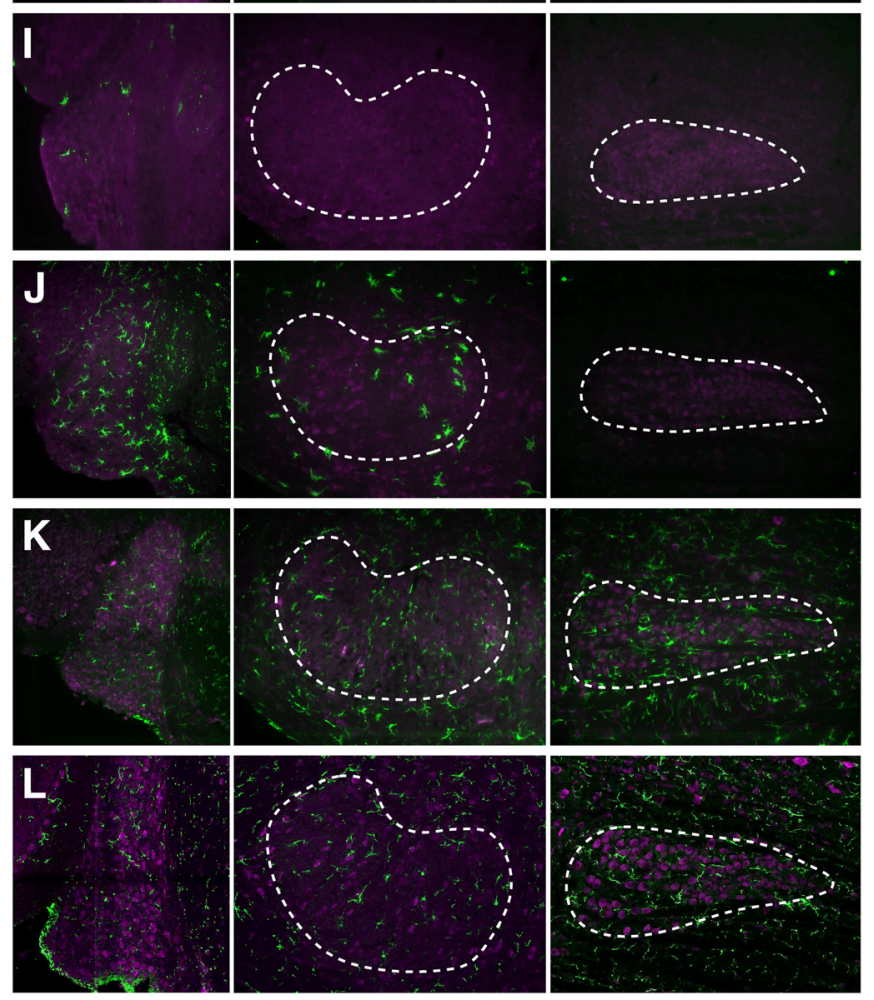

the rendering was adjusted manually to achieve an accurate RDA fill. Calyceal surface area as well as volume were recorded and used to calculate the shape factor of calyces from monoinnervated neurons. A shape factor was calculated as $(\mathrm{SA} 1.5) /(6 \sqrt{\pi} \mathrm{V})$ where $\mathrm{SA}$ is the surface area of the calyx and V is its volume (DeHoff, 1978; Muniak et al., 2018). A shape factor of 1.0 characterizes a perfect sphere. A large shape factor corresponds with 
Table 1: List of antibodies used in the project

\begin{tabular}{lllll}
\hline Antigen & Host & RRID & Catalog number & Source \\
\hline Primary antibodies & Chicken & AB_304558 & ab4674 & Dilution \\
GFAP & Rabbit & AB_839504 & 019-19741 & Wako \\
IBA1 & Rabbit & AB_2285905 & 135503 & Synaptic Systems \\
VGluT1/2 & & & The00 \\
Secondary antibodies & Chicken & AB_2534096 & A11039 & Thermo Fisher \\
Alexa Fluor 488 & Rabbit & AB_2535812 & A21244 & Thermo Fisher \\
Alexa Fluor 647 & & & & $1: 200$ \\
& & &
\end{tabular}

more complex structure, which, in the case of calyx of Held, represents its intricate branching. The analysis was performed blind to the animal treatment group.

\section{Determination of monoinnervation and polyinnervation}

The monoinnervation or polyinnervation status of MNTB neurons was evaluated during the reconstruction of RDAfilled calyces of Held. Neurons were classified as monoinnervated if the neuron was contacted only by a single RDA-labeled calyx, with no VGLUT1/2 immunolabel seen outside this calyx. Neurons were classified as polyinnervated if VGLUT1/2 immunolabel was present on the MNTB cell outside of the RDA-filled calyx. To be considered additional calyceal input, the VGLUT1/2 immunolabel outside of the RDA-filled calyx covered at least $25 \%$ of the MNTB neuron surface. The threshold of $25 \%$ was chosen because the smallest RDA-filled calyx detected across all the 10 analyzed animals contacted $\sim 25 \%$ of the neuronal surface area (Fig. 2). We then determined the percentage of analyzed MNTB neurons that were polyinnervated for the control and BLZ945-treated groups (Table 2).

\section{Auditory brainstem recordings (ABRs)}

ABRs were performed on 13 control and 14 BLZ945treated mice at four weeks and on 11 control and 15
BLZ945 injected animals at seven weeks. Mice were anesthetized with an intramuscular injection of ketamine (75 mg/kg, KetaVed, VEDCO) and xylazine $(15 \mathrm{mg} / \mathrm{kg}$, AnaSed, NADA\# 139-236). Body temperature was maintained at $35^{\circ} \mathrm{C}$ via a far infrared warming pad (Kent Scientific, RT-0501). Sterile ocular lubricant (Puralube Vet Ointment, 006PHM02-1-8) was applied on the eyes following ketamine/xylazine injection. Three pin electrodes were inserted subcutaneously. The positive, negative and ground electrodes were positioned at the vertex, the right cheek, and in the back near the right leg, respectively. Electrodes were connected to a Tucker-Davis Technologies (TDT) RA4PA 4-channel Medusa amplifier, which was connected to a TDT RA16 Medusa Base Station.

ABRs were performed in a sound-attenuating chamber (102 × $98 \times 81 \mathrm{~cm}$, Industrial Acoustics Company). Sound stimuli were generated using TDT SigGen software version 4.4 and presented 500 times at a rate of 21 stimuli per second using TDT MF1 Multi-Function Speaker through an ear tube inserted in the animal's left ear. Acoustic stimuli were emitted via a TDT RP2.1 enhanced real time processor. We controlled the sound level via a TDT PA5 programmable attenuator. Recorded responses were amplified by a TDT SA1 stereo power amp, filtered with the control of BioSig software version 4.4. ABRs were recorded for $12 \mathrm{~ms}$ in response to 100 - $\mu$ s click stimuli or 3-ms pure tone stimuli $(4,8,12,16,24,32 \mathrm{kHz})$ at decreasing sound levels (5 dB SPL steps from 80 to $10 \mathrm{~dB}$

Table 2: Numbers of calyces of Held analyzed and polyinnervated neurons

\begin{tabular}{|c|c|c|c|c|c|c|}
\hline Treatment & $\begin{array}{l}\text { Animals } \\
\text { used }\end{array}$ & $\begin{array}{l}\text { Age } \\
\text { (weeks) }\end{array}$ & Animal ID & $\begin{array}{l}\text { Reconstructed } \\
\text { calyces }\end{array}$ & $\begin{array}{l}\text { Number of } \\
\text { polyinnervated neurons }\end{array}$ & $\begin{array}{l}\% \text { of polyinnervated } \\
\text { neurons }\end{array}$ \\
\hline \multirow[t]{4}{*}{ Control } & 4 & 3 & B987 & $\overline{9}$ & 1 & 11.11 \\
\hline & & & B989 & 8 & 0 & 0 \\
\hline & & & B991 & 13 & 0 & 0 \\
\hline & & & B994 & 7 & 2 & 28.57 \\
\hline \multirow[t]{4}{*}{ BLZ945 } & 4 & & B990 & 9 & 5 & 55.56 \\
\hline & & & B992 & 9 & 1 & 11.11 \\
\hline & & & B993 & 13 & 5 & 38.46 \\
\hline & & & B995 & 10 & 5 & 50 \\
\hline \multirow[t]{5}{*}{ Control } & 5 & 4 & B886 & 8 & 0 & 0 \\
\hline & & & B887 & 7 & 1 & 14.29 \\
\hline & & & B888 & 8 & 0 & 0 \\
\hline & & & B894 & 9 & 0 & 0 \\
\hline & & & B896 & 9 & 1 & 11.11 \\
\hline \multirow[t]{5}{*}{ BLZ945 } & 5 & & B889 & 14 & 4 & 28.57 \\
\hline & & & B890 & 10 & 3 & 30 \\
\hline & & & B891 & 13 & 1 & 7.69 \\
\hline & & & B895 & 14 & 0 & 0 \\
\hline & & & B897 & 12 & 2 & 16.67 \\
\hline
\end{tabular}




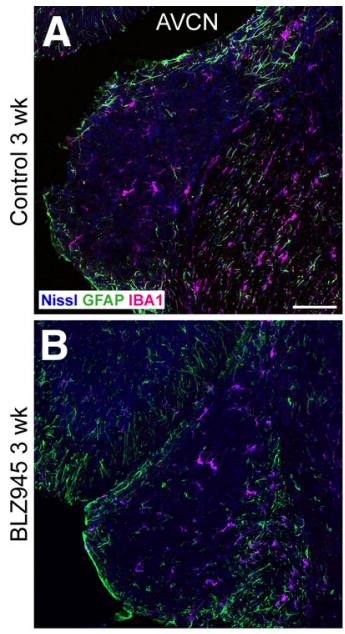

Niss
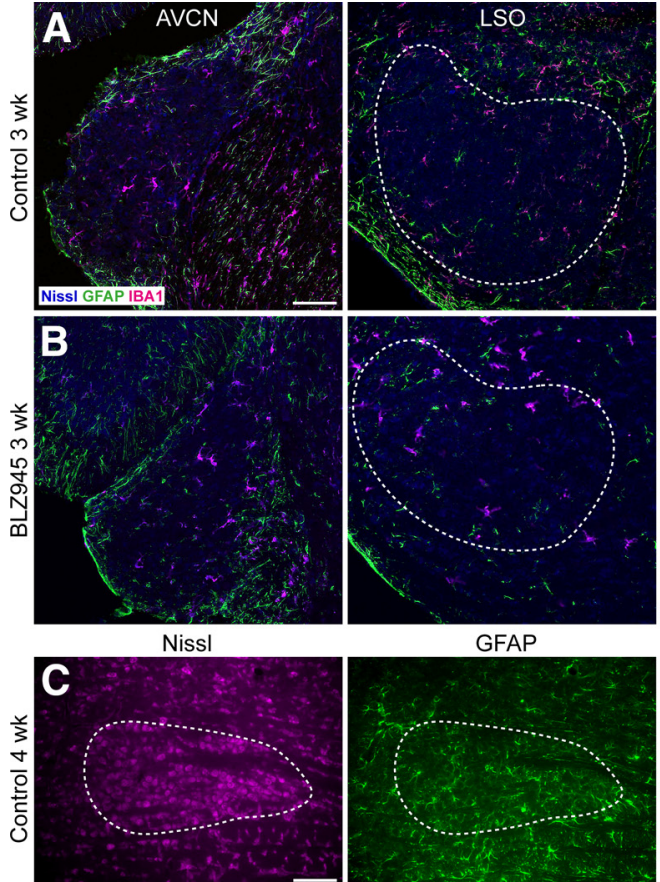

GFAP
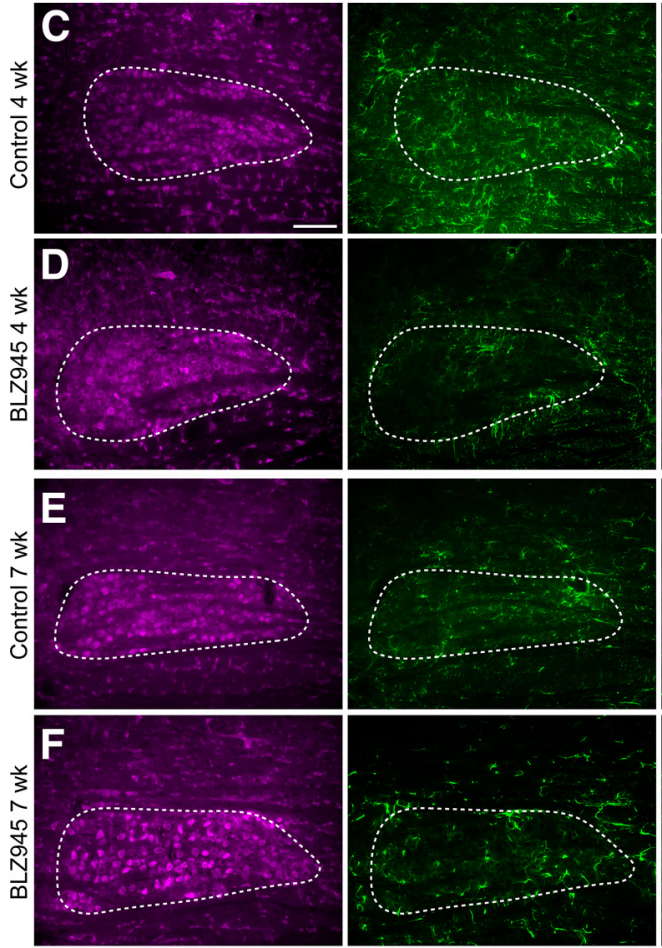

$$
\text { G }
$$

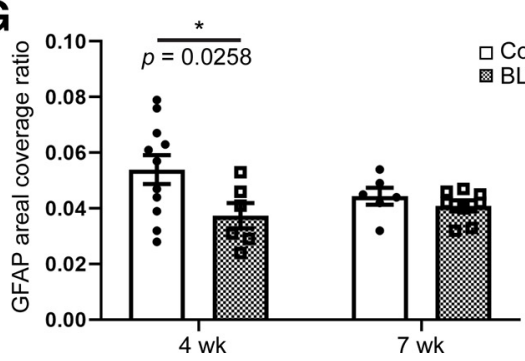

Figure 2. Effects of BLZ945 treatment withdrawal on GFAP expression in the auditory brainstem. Row A, Images of GFAP (green) and IBA1 (magenta) immunolabel in AVCN, LSO, and MNTB in control mice at three weeks. Dashed lines indicate LSO and MNTB. GFAP expression was dense along outer edges of AVCN but sparse within the nucleus. GFAP expression was also sparse in LSO, but denser staining was observed in MNTB. Row B, Images of GFAP and IBA1 immunolabel in AVCN, LSO, and MNTB in BLZ945treated mice at three weeks. GFAP immunolabel was seen along the outer edges of AVCN and was sparse in LSO. Some GFAP immunoloabel was seen in MNTB. Row $\boldsymbol{C}$, Images of Nissl, GFAP immunolabel and an overlay of both in MNTB in control mice at four weeks. GFAP expression extended throughout the MNTB. Row $\boldsymbol{D}$, Images of Nissl, GFAP immunolabel and an overlay of both in MNTB in BLZ945-treated mice at four weeks. GFAP expression was denser than at three weeks but was localized more to the boundaries of MNTB. Row $\boldsymbol{E}, \boldsymbol{F}$, Images of MNTB in control and BLZ945-injected mice, respectively, at seven weeks. GFAP 
continued

immunolabeling was pronounced and found throughout MNTB. G, Areal coverage of GFAP expression in MNTB at four and seven weeks. GFAP expression was significantly decreased in BLZ945-treated animals at four weeks compared with age-matched controls. No difference was found between the two experimental groups at seven weeks, and there were no age-related changes in GFAP areal coverage ratio in control or BLZ945-injected mice. Scale bar in row $\boldsymbol{A}$ : $100 \mu \mathrm{m}$, applies to all panels in rows $\boldsymbol{A}$, $\boldsymbol{B}$. Scale bar in row $\boldsymbol{C}: 100 \mu \mathrm{m}$, applies to all panels in rows $\boldsymbol{C}-\boldsymbol{F}$; ${ }^{*} p<0.05$.

SPL). An averaged response was computed at each sound level and used for analysis.

\section{ABR analysis}

ABRs were analyzed for threshold, peak latency, interpeak latency, and peak amplitude. We computed the mean noise level from the first 20 data points, before the ABR trace. Threshold was defined as the lowest sound level at which the peak I level $(\mu \mathrm{V})$ was $\geq 4$ SDs above the noise level (Bogaerts et al., 2009). Peaks were manually determined by a blinded observer, and data were recorded to a spreadsheet in BioSig from which the following analyses were performed. Absolute peak latency was determined as the time period from the sound onset to the apex of the peak. Interpeak latency was measured by calculating the difference between absolute peak latencies between peaks I-II, II-III, III-IV, I-III, and I-IV. Peak amplitude was determined as the change in microvolts $(\mu \mathrm{V})$ between the preceding trough and apex of the peak. Simple linear regressions of input-output functions of absolute peak latencies, interpeak latencies, and trough-topeak amplitudes at recorded frequency and intensity levels were analyzed. Input-output functions were only analyzed if at least three animals per group were at or above threshold (Tables 3, 4, 5).

\section{Experimental design and statistical analysis}

We used multiple litters for each experimental group. Each litter contained pups injected with DMSO or BLZ945 to account for the variability between litters. Quantitative results are presented as mean scores \pm SEM. Statistical analysis was done using Prism Software (v8.4.1; GraphPad Software). Comparisons between different treatment (control and BL7945) and age groups (three and four weeks or four and seven weeks) were made using two-way ANOVA with Sidak's multiple comparisons test unless otherwise indicated. Statistical significance was accepted at $p<0.05$. Details of statistical analyses are presented in Tables 3-5.

\section{Results}

\section{Microglia repopulate the MNTB after cessation of treatment}

Treatment with BLZ945 early in development (repeated subcutaneous injections at P2, P4, P6, P8, and P10) was used to eliminate microglia in the brainstem of mice as previously described (Milinkeviciute et al., 2019). We first determined whether microglia return after treatment cessation and if so, to what extent and over what time course. We perfused mice at several posttreatment time points: P14, P18, three, four, and seven weeks (Fig. 1A). We used four DMSO and 4 BLZ945-injected mice at P14, three DMSO and 4 BLZ945-treated mice at P18, five DMSO and five BLZ945-treated mice at three weeks, six DMSO and six BLZ945-treated mice at four weeks and four DMSO and seven BLZ945-injected mice at seven weeks. Littermate controls injected with DMSO were used in each age group. Each cohort consisted of animals from a few different litters to account for the variability between litters. Microglia were identified using IBA1 immunofluorescence. The areal coverage of IBA1 immunolabel was used to evaluate the extent of microglia repopulation at four and seven weeks (Fig. 1B). There was no significant difference in the IBA1 areal coverage in the MNTB (four-week DMSO: $0.06 \pm 0.004$; four-week BLZ945: $0.05 \pm 0.01$; seven-week DMSO: $0.04 \pm 0.01$; seven-week BL7945: $0.04 \pm 0.003)$ between control and drug treated animals at both four $(p=0.0574, t=2.360 ; \mathrm{df}=19)$ and seven $(p=0.9113, t=$ $0.3882 ; \mathrm{df}=19)$ weeks. IBA1 immunolabeling significantly decreased with age only in control animals (DMSO: $p=$ 0.0166, $t=2.943$; $d f=19$; BLZ945: $p=0.8425, t=0.5286$; $\mathrm{df}=19$ ), but there was no difference between DMSO controls and BLZ945-treated mice at seven weeks $(p=0.9113$, $t=0.3882 ; \mathrm{df}=19$; Fig. $1 B$ ).

Microglia densely populated VCN, LSO, and MNTB of control mice in each age group (Fig. 1C-G). In BLZ945-injected mice, microglia were not observed in the VCN, LSO or MNTB at P14 (Fig. 1H). A few microglial cells were seen in the VCN at P18 but no IBA1 staining was observed in the LSO or MNTB (Fig. 1/). By three weeks, microglia were seen throughout VCN and in LSO, but not in MNTB (Fig. 1J). At four and seven weeks, microglia were present in all three auditory brainstem nuclei (Fig. $1 K, L)$. This gradual microglial repopulation, first in VCN, then LSO, then MNTB reflects an overall lateral-to-medial movement of microglial emergence that is seen throughout the brainstem in our sections, reflecting a similar trend to how microglia occupy the brainstem during the first two postnatal weeks (Dinh et al., 2014). Thus, microglial repopulation of MNTB takes place between the ages of three and four weeks, or 11-18 d after the treatment is discontinued.

\section{GFAP expression in the MNTB returns to control levels after return of microglia}

GFAP is regarded as a marker of mature (Wofchuk and Rodnight, 1995; Gomes et al., 1999; Middeldorp and Hol, 2011) or active astrocytes (Eng and Ghirnikar, 1994; Fuentes-Santamaria et al., 2017). In MNTB, GFAP expression changes during development, with no expression at $\mathrm{P0}$, very sparse expression at P6, and increased density at P14. GFAP staining in VCN is first observed at P14 and some positive fibers are seen in MNTB at P23 (Dinh et al., 2014). We looked at $20 \times$ confocal images of sections 
Table 3: Descriptive statistics of immunostaining and confocal data

\begin{tabular}{ll}
\hline Analysis & Mean \pm SEM \\
\hline IBA1 (two-way ANOVA, & 4 weeks: \\
$\begin{array}{l}\text { Sidak's multiple compari- } \\
\text { sons test) }\end{array}$ & Control 0.06 $0.004(n=6)$, BLZ945 0.05 $\pm 0.01(n=6) ;$ \\
& Control $0.04 \pm 0.01(n=4)$, \\
& BLZ945 0.04 $0.003(n=7)$ \\
& \\
& \\
GFAP (two-way ANOVA, & 4 weeks: \\
Sidak's multiple compari- & Control $0.05 \pm 0.005(n=11$, BLZ945 $0.04 \pm 0.005(n=6) ;$ \\
sons test) & 7 weeks: \\
& Control $0.04 \pm 0.003(n=6)$, \\
& BLZ945 $0.04 \pm 0.002(n=8)$
\end{tabular}

Calyx of Held surface area (two-way ANOVA, Sidak's multiple comparisons test)

Polyinnervatioon of MNTB neurons

(two-way ANOVA, Sidak's multiple comparisons test)

Calyceal shape factor analysis

(two-way ANOVA, Sidak's

multiple comparisons test)
3 weeks:

Control $687.5 \pm 35.70(n=4)$, BLZ945 680.1.8 $\pm 13.40(n=4)$; 4 weeks:

Control 747.1 $\pm 21.19(n=5)$, BLZ945 707.8 $\pm 56.78(n=5)$

3 weeks:

Control $9.92 \pm 6.75(n=4)$, BLZ945 $38.78 \pm 9.89(n=4)$;

4 weeks:

Control $5.08 \pm 3.15(n=5)$, BLZ945 $16.59 \pm 5.82(n=5)$
Treatment

4-week Control-BLZ945:

$p=0.0574, t=2.36, \mathrm{df}=19$

7-week Control-BLZ945:

$p=0.9113, t=0.388, \mathrm{df}=19$;

Control 4-7 weeks:

$p=0.0166, t=2.943, \mathrm{df}=19$;

BLZ945 4-7 weeks:

$p=0.8425, t=0.529, \mathrm{df}=19$

4-week Control-BLZ945:

$p=0.0258, t=2.66, \mathrm{df}=27$;

7-week Control-BLZ945:

$p=0.8450, t=0.522, \mathrm{df}=27$;

Control 4-7 weeks:

$p=0.2535, t=1.537, \mathrm{df}=27$;

BLZ945 4-7 weeks:

$p=0.8381, t=0.534, \mathrm{df}=27$

3-week Control-BLZ945:

$p=0.9895, t=0.1310, \mathrm{df}=14$; 4-week Control-BLZ945:

$p=0.7005, t=0.7724, \mathrm{df}=14$;

Control 3-4 weeks:

$p=0.4941, t=1.103, \mathrm{df}=14$;

BLZ945 3-4 weeks:

$p=0.8527, t=0.5126, \mathrm{df}=14$

3-week Control-BLZ945:

$p=0.0191, t=2.998, \mathrm{df}=14$;

4-week Control-BLZ945:

$p=0.3645, t=1.336, d f=14$;

Control 3-4 weeks:

$p=0.8435, t=0.53, \mathrm{df}=14$;

BLZ945 3-4 weeks:

$p=0.0574, t=2.43, \mathrm{df}=14$

3-week Control-BLZ945:

$p=0.3210, t=1.425, \mathrm{df}=14$;

4-week Control-BLZ945:

$p=0.2488, t=1.594, \mathrm{df}=14$;

Control 3-4 weeks:

$p=0.3715, t=1.323, \mathrm{df}=14$;

BLZ945 3-4 weeks:

$p=0.3717, t=1.322, \mathrm{df}=14$ through the VCN, LSO, and MNTB stained for IBA1 and GFAP at three weeks (11 d postcessation of treatment) in three DMSO and three BLZ945-treated mice. As noted above, microglia repopulated auditory brainstem sequentially, starting from the most peripheral nuclei. At three weeks, microglia were found throughout the VCN, LSO, and MNTB of control mice. In VCN, GFAP labeling was mostly confined to outer layer of the nucleus with sparse labeling in the deeper layers. In LSO, GFAP-positive cells were also sparse while MNTB appeared to be the most populated (Fig. 2A). Similar to control animals, in BLZ945injected animals, microglia were spread throughout the VCN, however, in the LSO fewer cells were observed, and they appeared larger than in the VCN or LSO of control animals. MNTB was still devoid of IBA1-positive cells. In VCN GFAP was largely restricted to outer layers. GFAP staining in LSO was also very similar to that of control mice. In MNTB, GFAP-positive cells were observed throughout the nucleus (Fig. 2B). We showed that microglia fully repopulated MNTB by the age of four weeks, thus, next we focused on GFAP expression at four and seven weeks.

When microglia were depleted early postnatally, GFAP areal coverage was significantly reduced at P13 (Milinkeviciute et al., 2019). We examined GFAP areal immunofluorescence coverage ratio in 11 control and six BLZ945-treated mice at four weeks as well as six control and eight BLZ945-injected animals at seven weeks. Abundant GFAP was observed in four-week-old control mice (four-week DMSO: $0.05 \pm 0.01$; Fig. $2 C$ ). In drug treated four-week animals GFAP expression (fourweek BLZ945: $0.04 \pm$ 0.01; Fig. $2 D$ ) was localized more 
to the peripheral parts of the MNTB as reported in Milinkeviciute et al. (2019) at P13 while in control tissue GFAP labeling was found throughout the MNTB (Fig. 2A). At seven weeks, both, control and BLZ945-treated mice showed abundant GFAP labeling throughout the MNTB (seven-week DMSO: $0.04 \pm 0.003$; seven-week BLZ945: $0.04 \pm 0.002$ (Fig. $2 E-G$ ). The areal GFAP coverage was significantly reduced in BLZ945 injected mice at four weeks $(p=0.0258, t=2.66$; $d f=27)$ but was comparable to control levels at seven weeks $(p=0.8450$, $t=0.5215$; $d f=27$; Fig. $2 G$ ). There was no significant age-related change in GFAP areal coverage ratio in any of the two experimental groups (DMSO: $p=0.2535, t=1.537$; $\mathrm{df}=27$; BLZ945: $p=0.8381, t=0.5341$; $d f=27)$. These results suggest a relationship between microglia and the GFAP-positive astrocytic population in MNTB.

\section{MNTB neurons are monoinnervated after microglia recovery}

We labeled calyces of Held in the MNTB on both sides by injecting RDA into the VAS at the midline. Tissue was labeled with VGLUT1/2 antibody to identify excitatory inputs, which in MNTB mainly corresponds to calyces of Held (Billups, 2005). MNTB neurons contacted by an RDA labeled calyx were used for confocal imaging and subsequent reconstruction in $3 \mathrm{D}$ as previously described in Milinkeviciute et al. (2019). We reconstructed and analyzed calyces of Held from four DMSO and four BLZ945 at three weeks and five DMSO and five BLZ945-injected mice at four weeks. Monoinnvervated and polyinnervated MNTB neurons were identified in both control and BLZ945-treated mice at three weeks (Fig. $3 A-D$ ) and four weeks (Fig. 3E-H). We evaluated each MNTB neuron for VGLUT1/2 labeled inputs outside the RDA-labeled calyx (see Materials and Methods) to determine the percentage of neurons contacted by more than one calyx (three-week DMSO: $9.92 \pm 6.75$; three-week BLZ945: $38.78 \pm 9.89$; four-week DMSO: $5.08 \pm 3.15$; four-week BLZ945: $16.59 \pm$ 5.82). At three weeks, the percentage of polyinnervated neurons was significantly increased in the BLZ945-treated mouse group compared with their age-matched controls $(p=0.0191, t=2.998 ; \mathrm{df}=14)$. We found no significant difference in polyinnervation of MNTB neurons between control and drug treated animals at four weeks $(p=0.3645, t=1.336$; $\mathrm{df}=14$; Fig. 3/) Numbers of animals and calyces analyzed are presented in Table 2. These results suggest that neurons in MNTB remain polyinnervated when microglia are absent from MNTB.

We measured the surface area and volume of RDA-positive and reconstructed calyces from monoinnervated neurons. Calyces in the MNTB of control and drug injected mice did not differ in size at three weeks $(p=0.9895, t=0.131 ; \mathrm{df}=14)$ or four weeks $(p=0.7005$, $t=0.7724 ; \mathrm{df}=14)$. There was no significant age-related change in calyx surface area in control or BLZ945-treated mice (DMSO: $p=0.4941, t=1.103$; $d f=14$; BLZ945: $p=0.8527, t=0.5126$; df=14; Fig. $3 J$ ). Complexity of calyces was measured by calculating a shape factor using the formula described in see Materials and Methods (Bogaerts et al., 2009; Muniak et al., 2018). Calyces did not become more complex with age (DMSO: $p=0.3715$, $t=1.323 ; \mathrm{df}=14 ; \mathrm{BLZ945}: p=0.3717, t=1.322 ; \mathrm{df}=14$; Fig. $3 K)$. There was no difference observed between the intricacy of calyceal branching between control and BLZ945-injected mice at three weeks $(p=0.3210, t=$ $1.425 ; \mathrm{df}=14)$ or at four weeks $(p=0.2488, t=1.594$; $\mathrm{df}=14$; Fig. $3 K$; Table 3 ). In summary, temporary microglial depletion and subsequent repopulation does not seem to affect the growth and morphologic maturation (in terms of branching complexity) of calyces of Held.

\section{Temporary postnatal microglia elimination alters ABRs}

Temporary microglia depletion results in elevated hearing thresholds

Auditory brainstem responses were measured in mice at four weeks ( $n=13$ DMSO, $n=14$ BLZ945) and seven weeks ( $n=11$ DMSO, $n=15$ BLZ945). Click and pure tone stimuli were presented to the left ear at decreasing intensities from $80 \mathrm{~dB}$ SPL to $10 \mathrm{~dB}$ SPL. Click and pure tone traces were analyzed for threshold, the lowest intensity at which peak I level $(\mu \mathrm{V})$ was equal to or exceeded the 4 SDs above the mean noise level (Fig. 4A). BLZ945-treated mice had significantly higher thresholds in response to clicks at both four and seven weeks (four weeks: $p<$ 0.0001 , df $=47$; seven weeks $p=0.0321$, df $=47$; one-way ANOVA with Tukey's multiple comparisons; Fig. 4B). Age did not affect thresholds in both treatment groups (control: $p=0.1884$, df = 47; BLZ945: $p=0.9999$, df = 47; oneway ANOVA with Tukey's multiple comparisons; Fig. 4B). At four weeks, BLZ945-treated mice displayed elevated thresholds in response to pure tones at 4,8 , and $12 \mathrm{kHz}$ (4 kHz: $p<0.0001$, df=24.89; $8 \mathrm{kHz}: p<0.0001$, df=24.80; $12 \mathrm{kHz}: p=0.0007, \mathrm{df}=23.65 ; 16 \mathrm{kHz}: p=0.2228, \mathrm{df}=23.98$; $24 \mathrm{kHz}: p=0.2179$, df=16.58; $32 \mathrm{kHz}: p=0.3584$, df=24.08; Fig. 4B). At seven weeks, BLZ945-treated mice displayed pure tone thresholds that were comparable to control, and there were no significant differences at any frequency tested (4 kHz: $p=0.1043$, df $=24.0 ; 8 \mathrm{kHz}: p=0.4413$, df $=23.63$; $12 \mathrm{kHz}: p=0.8559, \mathrm{df}=24.0 ; 16 \mathrm{kHz}: p=0.4524$, df $=20.73$; $24 \mathrm{kHz}: p=0.8303$, df=20.54; $32 \mathrm{kHz}: p=0.9994$, df=22.56; Fig. 4C). Example traces indicating thresholds from each group at low and high frequencies are displayed in Figure 4D. These results indicate a temporary hearing impairment as a result of CSF1R inhibition during development and recovery after a prolonged period of microglial repopulation.

\section{Peak latencies are increased in BLZ945-treated animals}

Peaks in the ABR waveform reflect neuronal activity in different parts of the auditory pathway. Peak I indicates activity in the cochlea, spiral ganglion neurons (SGN), and VIIIth nerve, peak II represents, the cochlear nucleus $(C N)$, peak III, the superior olivary complex (SOC), and peak IV, the lateral lemniscus (LL; Jewett et al., 1970; Jewett and Williston, 1971; Picton et al., 1974; Henry, 1979). Mice were tested at four and seven weeks to compare the auditory function at early and late time points after microglial repopulation. All ABR statistical analyses including inputoutput simple linear regressions are displayed in Tables 4, 5 . 

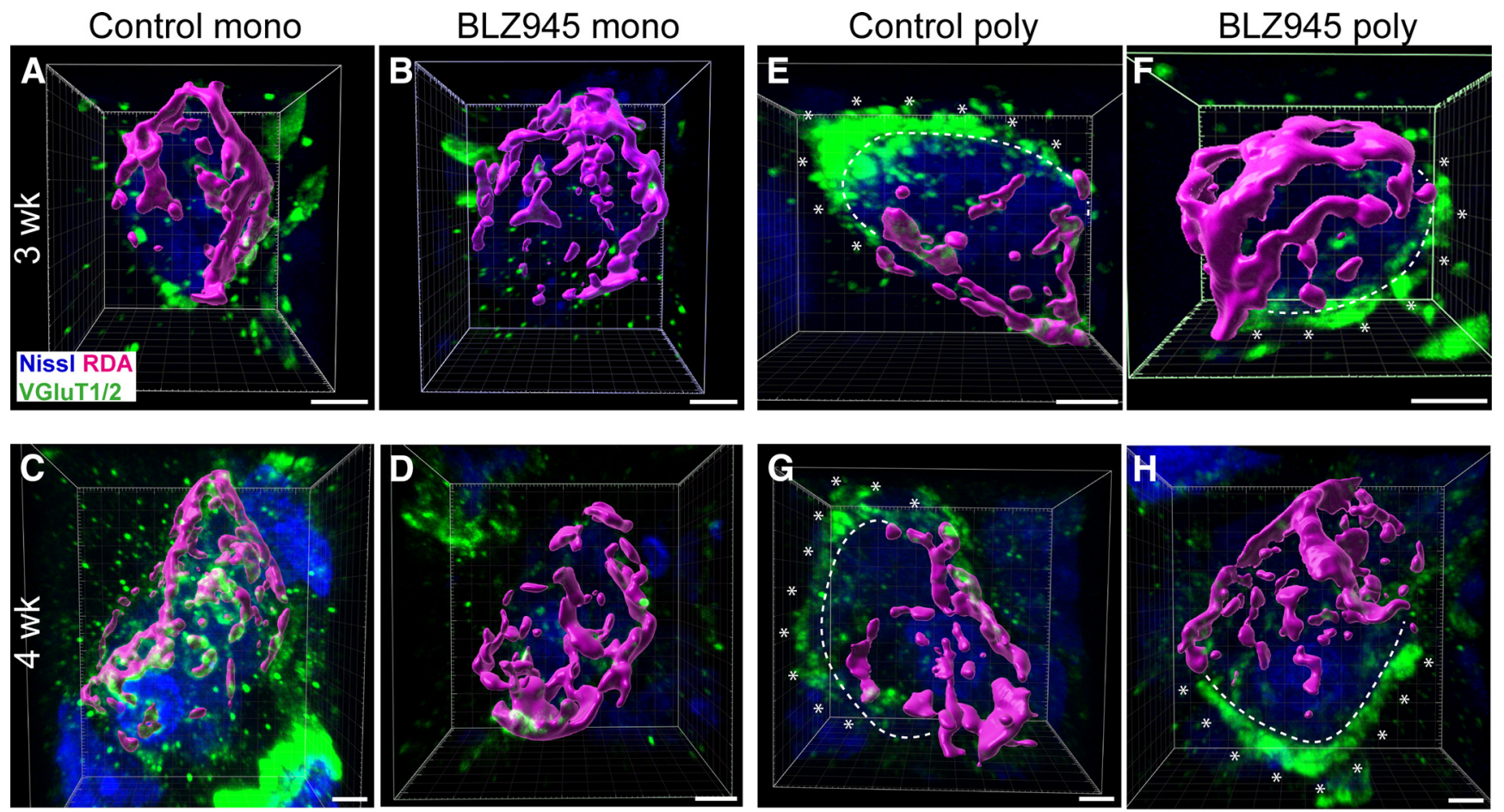
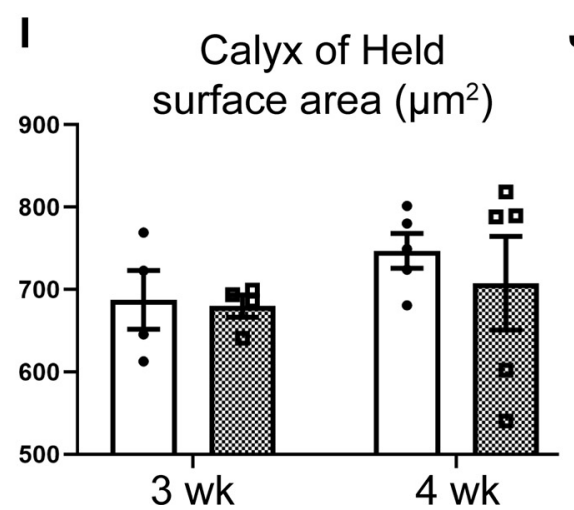

$\mathbf{J} \%$ of polyinnervated neurons

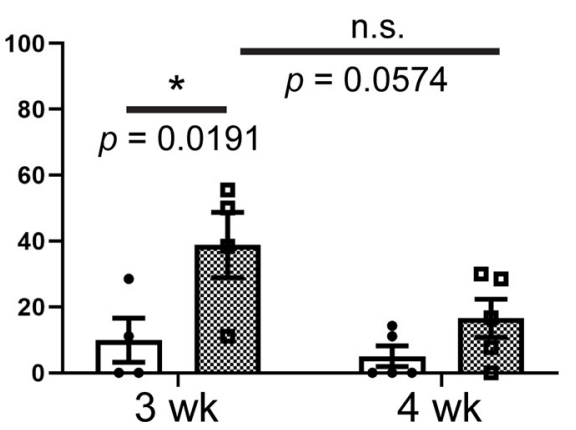

K Calyceal shape factor

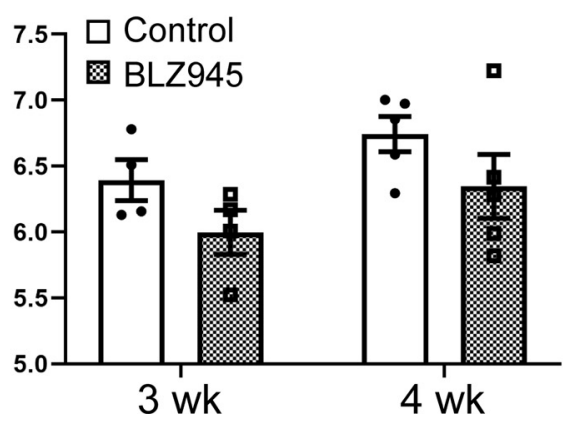

Figure 3. Confocal analysis of MNTB neurons. A, Monoinnervated neuron from a three-week-old control mouse. Reconstructed RDA-labeled calyx of Held (magenta); MNTB neuron (blue); and VGLUT1/2 labeling (green). B, Monoinnervated neuron from a threeweek-old BLZ945-treated mouse. C, $\boldsymbol{D}$, Monoinnervated neuron from a four-week-old control and BLZ945-injected mouse, respectively. $\boldsymbol{E}, \boldsymbol{F}$, Polyinnervated neuron from a three-week-old control and BLZ945-injected mouse, respectively. Dashed line indicates the contour of the neuron while asterisks show the VGLUT1/2-positive terminal contacting the same neuron. $\mathbf{G}, \boldsymbol{H}$, Polyinnervated neuron from a four-week-old control and BLZ945-treated animal, respectively. An extra calyceal input was determined by a continuous VGLUT1/2 staining outside of the RDA-positive area (asterisks) surrounding the MNTB neuron. I, Comparison of calyx of Held surface area in control and BLZ945-treated mice at three and four weeks. There was no difference between the two animal groups at both ages tested. No age-related increase in calyceal area was observed. $\boldsymbol{J}$, Comparison of polyinnervated MNTB neurons as a percentage of MNTB neurons analyzed revealed significantly more polyinnervated neurons in BLZ945-injected mice at three weeks when compared with controls. No difference between control and BLZ945 injected mice was found at four weeks. The age-related change in BLZ945-injected animals did not reach significance $(p=0.0574)$. Scale bars: $5 \mu \mathrm{m}(\boldsymbol{A}, \boldsymbol{B}, \boldsymbol{E}, \boldsymbol{F}), 3 \mu \mathrm{m}(\boldsymbol{C}, \boldsymbol{G}, \boldsymbol{H})$, and $4 \mu \mathrm{m}$ (D).

Peak latency was determined as the time (ms) from stimulus at which the apex of the peak was detected (Fig. 4A). At four weeks, BLZ945-treated mice showed significantly increased peak I latencies in lower and middle frequencies. Higher frequencies displayed significantly decreased peak I latencies (Fig. 5A). At seven weeks, treatment effects on peak I latencies were still observed at the lower and middle frequencies, but no differences were seen at the higher frequencies (Fig. 5A), indicating some recovery in peak I latencies.
Peak II latency in BLZ945-treated mice was significantly increased in low and middle frequencies but significantly decreased in high frequencies at four weeks (Fig. 5B). By seven weeks, peak II latency remained significantly delayed in the lower and middle frequencies but recovered at high frequencies (Fig. 5B). BLZ945 treatment led to significantly increased peak III latency (Fig. 5C) in most frequencies tested at four weeks. These treatment effects remained at some frequencies at seven weeks, but multiple comparison tests did not 


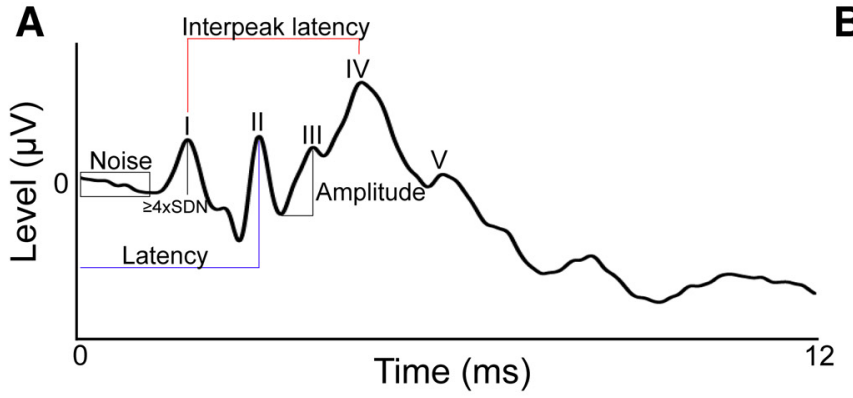

D

$8 \mathrm{kHz}$

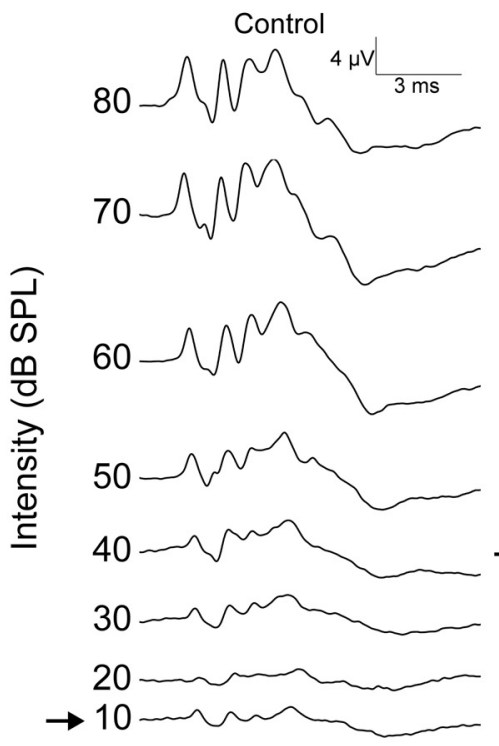

B

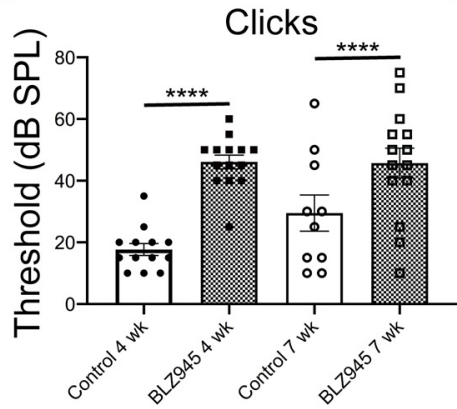

C

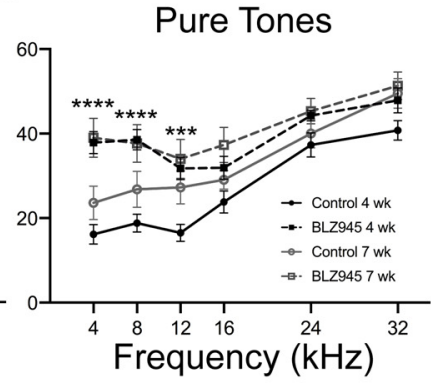

7 wk

\section{BLZ945 \\ Control}

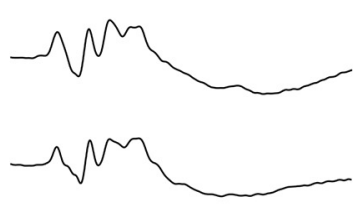

BLZ945

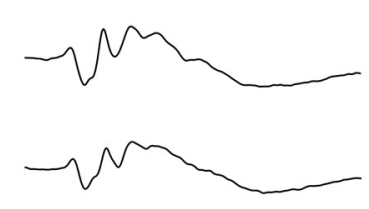

$32 \mathrm{kHz}$

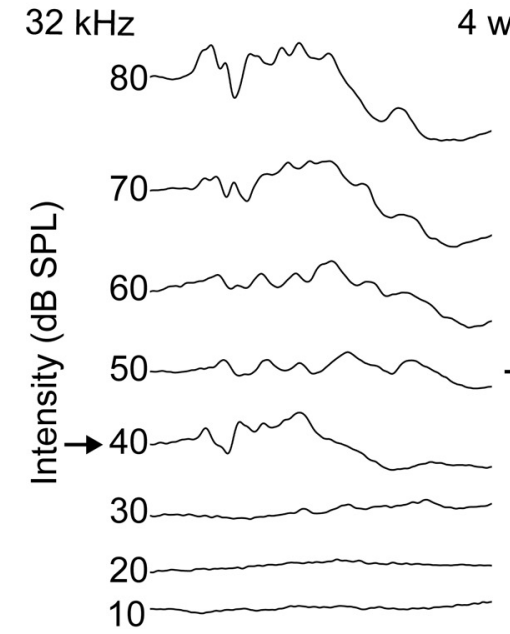

$4 w k$
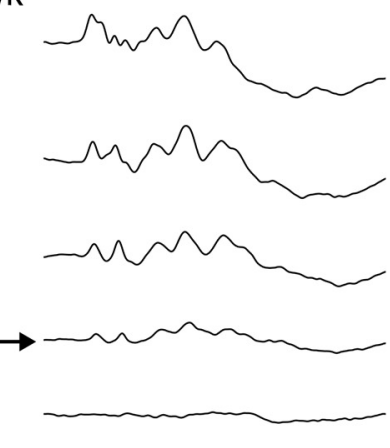
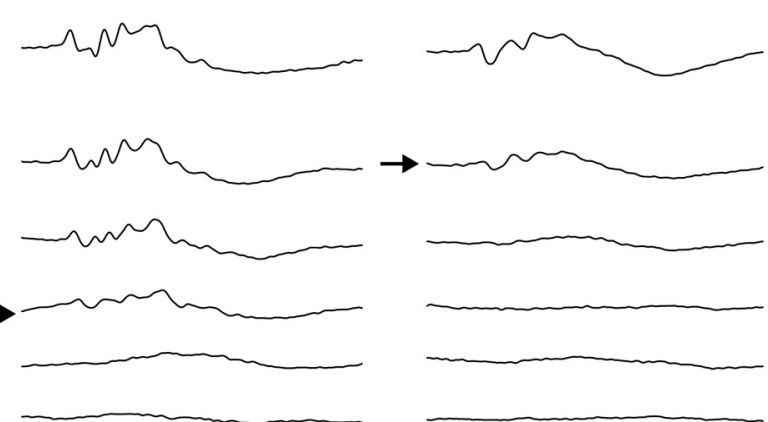

$7 \mathrm{wk}$
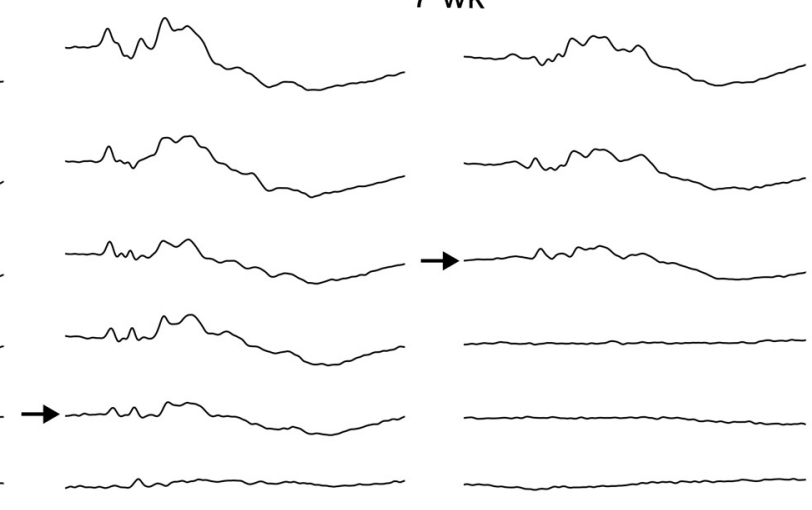

Figure 4. Effect of temporary BLZ945 treatment on auditory thresholds. A, Example trace for method of ABR analysis. Threshold was determined as the sound intensity for which peak I amplitude was greater than or equal to SDs above the averaged noise before the beginning of the ABR trace. Latency (blue) is the time of the apex of the peak from stimulus onset. Interpeak latency (red) is the difference in time between two respective peaks. Amplitude (black) is the difference in microvolts between the preceding trough to the apex of the peak. $\boldsymbol{B}$, Averaged thresholds for responses to click stimuli at four and seven weeks. BLZ945 injected mice had significantly elevated thresholds compared with their age-matched controls at four and seven weeks. $\boldsymbol{C}$, Averaged auditory thresholds for responses to pure tone stimuli at four weeks (black) and seven weeks (gray). BLZ945-treated animals had significantly elevated thresholds in response to 4, 8, and $12 \mathrm{kHz}$ at four weeks. Treatment did not affect thresholds at seven weeks, compared with age-matched controls. $\boldsymbol{D}$, Example traces in response to pure tones in control and BL7945-treated mice at four and seven weeks of age. Thresholds are indicated with black arrows. 
Table 4: Two-way ANOVA and simple linear regressions

\begin{tabular}{|c|c|c|c|c|c|c|c|c|c|c|c|c|}
\hline \multirow{3}{*}{$\begin{array}{c}\text { Stimulus/ } \\
\text { peak }\end{array}$} & \multicolumn{8}{|c|}{ Absolute peak latency (ms) } & \multicolumn{4}{|c|}{ Simple linear regression } \\
\hline & \multicolumn{2}{|c|}{ Mean \pm SEM } & \multicolumn{2}{|c|}{ Treatment } & \multicolumn{2}{|c|}{ Intensity } & \multicolumn{2}{|c|}{ Interaction } & \multicolumn{2}{|c|}{$\begin{array}{l}\text { Differences } \\
\text { between slopes }\end{array}$} & \multicolumn{2}{|c|}{$\begin{array}{c}\text { Differences between } \\
\text { elevations } \\
\text { or intercepts }\end{array}$} \\
\hline & 4 weeks & 7 weeks & 4 weeks & 7 weeks & 4 weeks & 7 weeks & 4 weeks & 7 weeks & 4 weeks & 7 weeks & 4 weeks & 7 weeks \\
\hline $\begin{array}{l}4 \mathrm{kHz} \\
\quad \text { peak I }\end{array}$ & $\begin{array}{l}\text { Control } 1.803 \pm 0.035 \\
\qquad(n=13) ; \mathrm{BLZ} 945 \\
2.011 \pm 0.044(n=14)\end{array}$ & $\begin{array}{l}\text { Control } 1.78 \pm 0.04(n=11) \\
\quad \text { BLZ945 } 1.93 \pm 0.03 \\
\quad(n=15)\end{array}$ & $p<0.0001$ & $p<0.0001$ & $p<0.0001$ & $p<0.0001$ & $p=0.7740$ & $p=0.4004$ & $p=0.0410$ & $p=0.1317$ & $\begin{array}{l}\text { Not possible } \\
\text { to test }\end{array}$ & $p<0.0001$ \\
\hline $\begin{array}{l}8 \mathrm{kHz} \\
\text { peak I }\end{array}$ & $\begin{array}{l}\text { Control 1.82 } \pm 0.036(n=13) \\
\qquad \text { BLZ945 } 1.94 \pm 0.04 \\
(n=14)\end{array}$ & $\begin{array}{l}\text { Control } 1.79 \pm 0.04(n=11) \\
\quad \text { BLZ945 } 1.87 \pm 0.03 \\
\quad(n=15)\end{array}$ & $p<0.0001$ & $p<0.0001$ & $p<0.0001$ & $p<0.0001$ & $p=0.7755$ & $p=0.6425$ & $p=0.1907$ & $p=0.1613$ & $p<0.0001$ & $p<0.0001$ \\
\hline $\begin{array}{l}12 \mathrm{kHz} \\
\text { peak I }\end{array}$ & $\begin{array}{l}\text { Control } 1.88 \pm 0.04(n=13) \\
\quad \text { BLZ945 } 1.95 \pm 0.04 \\
(n=14)\end{array}$ & $\begin{array}{l}\text { Control } 1.80 \pm 0.04(n=11) \\
\quad \text { BLZ945 } 1.87 \pm 0.03 \\
(n=15)\end{array}$ & $p<0.0001$ & $p<0.0001$ & $p<0.0001$ & $p<0.0001$ & $p=0.7517$ & $p=0.9777$ & $p=0.3589$ & $p=0.1551$ & $p<0.0001$ & $p<0.0001$ \\
\hline $\begin{array}{l}16 \mathrm{kHz} \\
\text { peak I }\end{array}$ & $\begin{array}{l}\text { Control } 1.89 \pm 0.04(n=13) \\
\quad \text { BLZ945 } 1.93 \pm 0.03 \\
(n=14)\end{array}$ & $\begin{array}{l}\text { Control } 1.77 \pm 0.03(n=11) \\
\quad \text { BLZ945 } 1.86 \pm 0.03 \\
(n=15)\end{array}$ & $p=0.0020$ & $p<0.0001$ & $p<0.0001$ & $p<0.0001$ & $p=0.1362$ & $p=0.9942$ & $p=0.0021$ & $p=0.5215$ & $\begin{array}{l}\text { Not possible } \\
\text { to test }\end{array}$ & $p<0.0001$ \\
\hline $\begin{array}{l}24 \mathrm{kHz} \\
\text { peak I }\end{array}$ & $\begin{array}{l}\text { Control 1.91 } \pm 0.04(n=13) \\
\quad \text { BLZ945 } 1.80 \pm 0.03 \\
(n=14)\end{array}$ & $\begin{array}{l}\text { Control } 1.76 \pm 0.03(n=11) \\
\quad \text { BLZ945 } 1.81 \pm 0.04 \\
(n=15)\end{array}$ & $p<0.0001$ & $p=0.1081$ & $p<0.0001$ & $p<0.0001$ & $p=0.8522$ & $p=0.9943$ & $p=0.0728$ & $p=0.5176$ & $p<0.0001$ & $p=0.1525$ \\
\hline $\begin{array}{l}32 \mathrm{kHz} \\
\text { peak I }\end{array}$ & $\begin{array}{l}\text { Control 1.91 } \pm 0.04(n=13) \\
\quad \text { BLZ945 } 1.81 \pm 0.03 \\
(n=14)\end{array}$ & $\begin{array}{l}\text { Control } 1.83 \pm 0.03(n=11) \\
\quad \text { BLZ945 } 1.85 \pm 0.03 \\
(n=15)\end{array}$ & $p=0.0029$ & $p=0.5552$ & $p=0.0946$ & $p=0.1688$ & $p=0.9998$ & $p=0.9872$ & $p=0.8675$ & $p=0.8108$ & $p=0.0014$ & $p=0.6973$ \\
\hline $\begin{array}{l}4 \mathrm{kHz} \\
\quad \text { peak II }\end{array}$ & $\begin{array}{l}\text { Control } 2.89 \pm 0.04(n=13) \\
\quad \text { BLZ945 } 3.18 \pm 0.05 \\
(n=14)\end{array}$ & $\begin{array}{l}\text { Control } 2.82 \pm 0.04(n=11) \\
\quad \text { BLZ945 } 2.99 \pm 0.03 \\
(n=15)\end{array}$ & $p<0.0001$ & $p<0.0001$ & $p<0.0001$ & $p<0.0001$ & $p=0.8598$ & $p=0.9661$ & $p=0.7283$ & $p=0.4145$ & $p<0.0001$ & $p<0.0001$ \\
\hline $\begin{array}{l}8 \mathrm{kHz} \\
\quad \text { peak II }\end{array}$ & $\begin{array}{l}\text { Control } 3.05 \pm 0.02(n=13) \\
\quad \text { BLZ945 } 3.11 \pm 0.04 \\
(n=14)\end{array}$ & $\begin{array}{l}\text { Control } 2.88 \pm 0.03(n=11) \\
\quad \text { BLZ945 } 2.99 \pm 0.04 \\
(n=15)\end{array}$ & $p=0.0190$ & $p=0.0011$ & $p<0.0001$ & $p<0.0001$ & $p=0.5803$ & $p>0.9999$ & $p=0.6072$ & $p=0.8238$ & $p=0.0475$ & $p=0.0006$ \\
\hline $\begin{array}{l}12 \mathrm{kHz} \\
\text { peak II }\end{array}$ & $\begin{array}{l}\text { Control } 3.09 \pm 0.04(n=13) ; \\
\quad \text { BLZ945 } 3.17 \pm 0.04 \\
(n=14)\end{array}$ & $\begin{array}{l}\text { Control } 2.87 \pm 0.04(n=11) \\
\quad \text { BLZ945 } 2.95 \pm 0.03 \\
\quad(n=15)\end{array}$ & $p=0.0003$ & $p=0.0334$ & $p<0.0001$ & $p=0.0002$ & $p=0.7647$ & $p=0.9898$ & $p=0.1824$ & $p=0.3472$ & $p=0.0003$ & $p=0.0089$ \\
\hline $\begin{array}{l}16 \mathrm{kHz} \\
\text { peak II }\end{array}$ & $\begin{array}{l}\text { Control } 3.05 \pm 0.04(n=13) \\
\quad \text { BLZ945 } 3.13 \pm 0.03 \\
\quad(n=14)\end{array}$ & $\begin{array}{l}\text { Control } 2.83 \pm 0.04(n=11) \\
\quad \text { BLZ945 } 2.98 \pm 0.04 \\
(n=15)\end{array}$ & $p=0.0024$ & $p<0.0001$ & $p<0.0001$ & $p<0.0001$ & $p=0.9859$ & $p=0.9995$ & $p=0.3160$ & $p=0.7315$ & $p=0.0019$ & $p<0.0001$ \\
\hline $\begin{array}{l}24 \mathrm{kHz} \\
\text { peak II }\end{array}$ & $\begin{array}{l}\text { Control } 3.02 \pm 0.06(n=13) \\
\quad \text { BLZ945 } 2.76 \pm 0.04 \\
(n=14)\end{array}$ & $\begin{array}{l}\text { Control } 2.75 \pm 0.02(n=11) ; \\
\quad \text { BLZ945 } 2.67 \pm 0.06 \\
(n=15)\end{array}$ & $p<0.0001$ & $p=0.1407$ & $p=0.0040$ & $p=0.3100$ & $p=0.6485$ & $p=0.7841$ & $p=0.0431$ & $p=0.0567$ & $\begin{array}{l}\text { Not possible } \\
\text { to test }\end{array}$ & $p=0.0080$ \\
\hline $\begin{array}{l}32 \mathrm{kHz} \\
\text { peak II }\end{array}$ & $\begin{array}{l}\text { Control } 2.90 \pm 0.09(n=13) \\
\quad \text { BLZ945 } 2.72 \pm 0.03 \\
(n=14)\end{array}$ & $\begin{array}{l}\text { Control } 2.72 \pm 0.03(n=11) \\
\quad \text { BLZ945 } 2.67 \pm 0.04 \\
(n=15)\end{array}$ & $p=0.0006$ & $p=0.4970$ & $p<0.0001$ & $p=0.9996$ & $p=0.4457$ & $p=0.6959$ & $p=0.0042$ & $p=0.0507$ & $\begin{array}{l}\text { Not possible } \\
\text { to test }\end{array}$ & $p=0.0752$ \\
\hline $\begin{array}{l}4 \mathrm{kHz} \\
\quad \text { peak III }\end{array}$ & $\begin{array}{l}\text { Control } 3.88 \pm 0.04(n=13) \\
\quad \text { BLZ945 } 4.19 \pm 0.06 \\
(n=14)\end{array}$ & $\begin{array}{l}\text { Control } 3.68 \pm 0.03(n=11) \\
\quad \text { BLZ945 } 3.76 \pm 0.02 \\
(n=15)\end{array}$ & $p<0.0001$ & $p=0.0641$ & $p<0.0001$ & $p=0.5772$ & $p=0.7320$ & $p=0.9884$ & $p=0.0285$ & $p=0.8082$ & $\begin{array}{l}\text { Not possible } \\
\text { to test }\end{array}$ & $p=0.0072$ \\
\hline $\begin{array}{l}8 \mathrm{kHz} \\
\text { peak III }\end{array}$ & $\begin{array}{l}\text { Control } 3.89 \pm 0.03(n=13) \\
\quad \text { BLZ945 } 4.12 \pm 0.06 \\
(n=14)\end{array}$ & $\begin{array}{l}\text { Control } 3.68 \pm 0.02(n=11) \\
\quad \text { BLZ945 } 3.78 \pm 0.02 \\
(n=15)\end{array}$ & $p<0.0001$ & $p=0.0179$ & $p<0.0001$ & $p=0.7081$ & $p=0.2898$ & $p>0.9999$ & $p=0.0109$ & $p=0.7434$ & $\begin{array}{l}\text { Not possible } \\
\text { to test }\end{array}$ & $p=0.0053$ \\
\hline $\begin{array}{l}12 \mathrm{kHz} \\
\text { peak III }\end{array}$ & $\begin{array}{l}\text { Control } 3.98 \pm 0.04(n=13) \\
\quad \text { BLZ945 } 4.21 \pm 0.05 \\
\quad(n=14)\end{array}$ & $\begin{array}{l}\text { Control } 3.71 \pm 0.02(n=11) \\
\quad \text { BLZ945 } 3.82 \pm 0.02 \\
\quad(n=15)\end{array}$ & $p<0.0001$ & $p=0.0113$ & $p<0.0001$ & $p=0.6296$ & $p=0.9039$ & $p=0.9884$ & $p=0.9176$ & $p=0.6784$ & $p<0.0001$ & $p=0.0018$ \\
\hline $\begin{array}{l}16 \mathrm{kHz} \\
\text { peak III }\end{array}$ & $\begin{array}{l}\text { Control } 4.02 \pm 0.06(n=13) \\
\quad \text { BLZ945 } 4.25 \pm 0.04 \\
(n=14)\end{array}$ & $\begin{array}{l}\text { Control } 3.72 \pm 0.02(n=11) \\
\quad \text { BLZ945 } 3.91 \pm 0.03 \\
(n=15)\end{array}$ & $p<0.0001$ & $p<0.0001$ & $p<0.0001$ & $p=0.6845$ & $p=0.8036$ & $p=0.9996$ & $p=0.0273$ & $p=0.7221$ & $\begin{array}{l}\text { Not possible } \\
\text { to test }\end{array}$ & $p<0.0001$ \\
\hline $\begin{array}{l}24 \mathrm{kHz} \\
\text { peak III }\end{array}$ & $\begin{array}{l}\text { Control } 3.98 \pm 0.05(n=13) \\
\quad \text { BLZ945 } 4.05 \pm 0.03 \\
\quad(n=14)\end{array}$ & $\begin{array}{l}\text { Control } 3.77 \pm 0.07(n=11) \\
\quad \text { BLZ945 } 3.80 \pm 0.05 \\
\quad(n=15)\end{array}$ & $p=0.1924$ & $p=0.7003$ & $p=0.0467$ & $p=0.1637$ & $p=0.9797$ & $p=0.9808$ & $p=0.2513$ & $p=0.7024$ & $p=0.1708$ & $p=0.4274$ \\
\hline $\begin{array}{l}32 \mathrm{kHz} \\
\text { peak III }\end{array}$ & $\begin{array}{l}\text { Control } 3.92 \pm 0.09(n=13) \\
\quad \text { BLZ945 } 4.07 \pm 0.03 \\
(n=14)\end{array}$ & $\begin{array}{l}\text { Control } 3.66 \pm 0.04(n=11) \\
\quad \text { BLZ945 } 3.88 \pm 0.02 \\
(n=15)\end{array}$ & $p=0.0060$ & $p=0.0114$ & ntinued) & $p=0.9482$ & $p=0.3418$ & $p=0.9991$ & $p=0.0023$ & $p=0.7152$ & $\begin{array}{l}\text { Not possible } \\
\text { to test }\end{array}$ & $p=0.0079$ \\
\hline
\end{tabular}


Table 4: Continued

\begin{tabular}{|c|c|c|c|c|c|c|c|c|c|c|c|c|}
\hline $\begin{array}{l}4 \mathrm{kHz} \text { peak } \\
\text { IV }\end{array}$ & $\begin{array}{l}\text { Control } 4.88 \pm 0.05(n=13) \\
\quad \text { BLZ945 } 5.36 \pm 0.07 \\
\quad(n=14)\end{array}$ & $\begin{array}{l}\text { Control } 4.62 \pm 0.06(n=11) \\
\quad \text { BLZ945 } 4.85 \pm 0.04 \\
(n=15)\end{array}$ & $p<0.0001$ & $p<0.0001$ & $p<0.0001$ & $p=0.0002$ & $p=0.9720$ & $p=0.5290$ & $p=0.1473$ & $p=0.5928$ & $p<0.0001$ & $p<0.0001$ \\
\hline $\begin{array}{c}8 \mathrm{kHz} \text { peak } \\
\text { IV }\end{array}$ & $\begin{array}{l}\text { Control } 4.92 \pm 0.05(n=13) \\
\quad \text { BLZ945 5.23 } \pm 0.06 \\
(n=14)\end{array}$ & $\begin{array}{l}\text { Control } 4.57 \pm 0.05(n=11) \\
\quad \text { BLZ945 } 4.86 \pm 0.03 \\
(n=15)\end{array}$ & $p<0.0001$ & $p<0.0001$ & $p<0.0001$ & $p=0.0024$ & $p=0.3804$ & $p=0.9688$ & $p=0.6358$ & $p=0.2588$ & $p<0.0001$ & $p<0.0001$ \\
\hline $\begin{array}{l}12 \mathrm{kHz} \\
\quad \text { peak IV }\end{array}$ & $\begin{array}{l}\text { Control } 4.96 \pm 0.06(n=13) \\
\quad \text { BLZ945 } 5.32 \pm 0.08 \\
(n=14)\end{array}$ & $\begin{array}{l}\text { Control } 4.62 \pm 0.05(n=11) \\
\quad \text { BLZ945 } 4.87 \pm 0.04 \\
(n=15)\end{array}$ & $p<0.0001$ & $p<0.0001$ & $p<0.0001$ & $p=0.0023$ & $p=0.9533$ & $p=0.9198$ & $p=0.3836$ & $p=0.7575$ & $p<0.0001$ & $p<0.0001$ \\
\hline $\begin{array}{l}16 \mathrm{kHz} \\
\quad \text { peak IV }\end{array}$ & $\begin{array}{l}\text { Control } 4.93 \pm 0.06(n=13) \\
\quad \text { BLZ945 } 5.32 \pm 0.06 \\
(n=14)\end{array}$ & $\begin{array}{l}\text { Control } 4.62 \pm 0.04(n=11) \\
\quad \text { BLZ945 } 4.90 \pm 0.04 \\
(n=15)\end{array}$ & $p<0.0001$ & $p<0.0001$ & $p<0.0001$ & $p=0.0020$ & $p=0.9930$ & $p=0.9933$ & $p=0.6512$ & $p=0.9863$ & $p<0.0001$ & $p<0.0001$ \\
\hline $\begin{array}{l}24 \mathrm{kHz} \\
\quad \text { peak IV }\end{array}$ & $\begin{array}{l}\text { Control } 5.01 \pm 0.08(n=13) \\
\quad \text { BLZ945 } 5.00 \pm 0.03 \\
(n=14)\end{array}$ & $\begin{array}{l}\text { Control } 4.66 \pm 0.08(n=11) \\
\quad \text { BLZ945 } 4.88 \pm 0.03 \\
(n=15)\end{array}$ & $p=0.8684$ & $p=0.0679$ & $p=0.0009$ & $p=0.2143$ & $p=0.6273$ & $p=0.9372$ & $p=0.0130$ & $p=0.2559$ & $\begin{array}{l}\text { Not possible } \\
\text { to test }\end{array}$ & $p=0.0048$ \\
\hline $\begin{array}{l}32 \mathrm{kHz} \\
\quad \text { peak IV }\end{array}$ & $\begin{array}{l}\text { Control } 5.02 \pm 0.13(n=13) \\
\quad \text { BLZ945 } 5.10 \pm 0.11 \\
(n=14)\end{array}$ & $\begin{array}{l}\text { Control } 4.62 \pm 0.05(n=11) \\
\quad \text { BLZ945 } 4.83 \pm 0.03 \\
\quad(n=15)\end{array}$ & $p=0.2854$ & $p=0.0060$ & $p<0.0001$ & $p=0.5935$ & $p=0.2226$ & $p=0.9938$ & $p=0.0027$ & $p=0.7382$ & $\begin{array}{l}\text { Not possible } \\
\text { to test }\end{array}$ & $p=0.0040$ \\
\hline & Mean & \pm SEM & erpeak latenc & (ms) & Inten & nsity & Intera & action & $\begin{array}{r}\text { Differ } \\
\text { betweer }\end{array}$ & $\begin{array}{l}\text { Simple I } \\
\text { ences } \\
\text { n slopes }\end{array}$ & $\begin{array}{r}\text { linear regressio } \\
\text { Differenc } \\
\text { eleva } \\
\text { inte }\end{array}$ & $\begin{array}{l}\text { es between } \\
\text { cens or }\end{array}$ \\
\hline Stimulus & 4 weeks & 7 weeks & 4 weeks & 7 weeks & 4 weeks & 7 weeks & 4 weeks & 7 weeks & 4 weeks & 7 weeks & 4 weeks & 7 weeks \\
\hline $\begin{array}{c}\mathrm{kHz} \\
\text { peak } \\
\mathrm{I}-\mathrm{II}\end{array}$ & $\begin{array}{l}\text { Control 1.04 } \pm 0.01(n=13) \\
\quad \text { BLZ945 } 1.08 \pm 0.02 \\
(n=14)\end{array}$ & $\begin{array}{l}\text { Control 1.05 } \pm 0.01(n=11) \\
\quad \text { BLZ945 } 1.06 \pm 0.01 \\
(n=15)\end{array}$ & $p=0.0153$ & $p=0.5483$ & $p=0.9959$ & $p=0.9992$ & $p=0.8222$ & $p=0.9891$ & $p=0.2560$ & $p=0.9111$ & $p=0.0206$ & $p=0.6128$ \\
\hline $\begin{array}{l}8 \mathrm{kHz} \\
\text { peak } \\
\mathrm{I}-\mathrm{II}\end{array}$ & $\begin{array}{l}\text { Control 1.22 } \pm 0.02(n=13) \\
\quad \text { BLZ945 } 1.17 \pm 0.02 \\
(n=14)\end{array}$ & $\begin{array}{l}\text { Control 1.09 } \pm 0.01(n=11) \\
\quad \text { BLZ945 } 1.11 \pm 0.01 \\
(n=15)\end{array}$ & $p=0.0140$ & $p=0.4202$ & $p=0.0731$ & $p=0.9810$ & $p=0.8531$ & $p=0.9632$ & $p=0.9154$ & $p=0.3888$ & $p=0.0028$ & $p=0.8857$ \\
\hline $\begin{array}{c}12 \mathrm{kHz} \\
\text { peak } \\
\mathrm{I}-\mathrm{II}\end{array}$ & $\begin{array}{l}\text { Control 1.22 } \pm 0.01(n=13) \\
\quad \text { BLZ945 1.22 } \pm 0.02 \\
(n=14)\end{array}$ & $\begin{aligned} n= & \text { Control } 1.09 \pm 0.01 \\
& (n=11) ; \text { BLZ945 } \\
& 1.10 \pm 0.01(n=15)\end{aligned}$ & $p=0.9395$ & $p=0.6103$ & $p=0.3768$ & $p=0.9975$ & $p=0.9098$ & $p=0.9748$ & $p=0.6346$ & $p=0.8061$ & $p=0.7079$ & $p=0.8426$ \\
\hline $\begin{array}{c}16 \mathrm{kHz} \\
\text { peak } \\
\mathrm{I}-\mathrm{II}\end{array}$ & $\begin{array}{l}\text { Control 1.12 } \pm 0.08(n=13) \\
\quad \text { BLZ945 } 1.15 \pm 0.01 \\
(n=14)\end{array}$ & $\begin{array}{l}\text { Control 1.06 } \pm 0.01(n=11) \\
\quad \text { BLZ945 } 1.12 \pm 0.01 \\
(n=15)\end{array}$ & $p=0.0653$ & $p=0.0671$ & $p=0.7001$ & $p=0.9954$ & $p=0.9939$ & $p=0.9911$ & $p=0.6778$ & $p=0.9407$ & $p=0.0770$ & $p=0.0770$ \\
\hline $\begin{array}{c}24 \mathrm{kHz} \\
\text { peak } \\
\mathrm{I}-\mathrm{II}\end{array}$ & $\begin{array}{l}\text { Control 1.06 } \pm 0.01(n=13) \\
\quad \text { BLZ945 } 0.94 \pm 0.02 \\
(n=14)\end{array}$ & $\begin{array}{l}\text { Control } 0.99 \pm 0.03(n=11) \\
\quad \text { BLZ945 } 0.86 \pm 0.02 \\
(n=15)\end{array}$ & $p=0.0001$ & $p=0.0028$ & $p=0.8792$ & $p=0.9741$ & $p=0.3152$ & $p=0.5636$ & $p=0.6021$ & $p=0.0421$ & $p<0.0001$ & $\begin{array}{l}\text { Not possible } \\
\text { to test }\end{array}$ \\
\hline $\begin{array}{c}32 \mathrm{kHz} \\
\text { peak } \\
\text { I-II }\end{array}$ & $\begin{array}{l}\text { Control } 0.92 \pm 0.04(n=13) \\
\quad \text { BLZ945 } 0.91 \pm 0.01 \\
\quad(n=14)\end{array}$ & $\begin{array}{l}\text { Control } 0.89 \pm 0.04(n=11) \\
\quad \text { BLZ945 } 0.82 \pm 0.01 \\
\quad(n=15)\end{array}$ & $p=0.7968$ & $p=0.0893$ & $p=0.2994$ & $p=0.6474$ & $p=0.3764$ & $p=0.1604$ & $p=0.0067$ & $p=0.0030$ & $\begin{array}{l}\text { Not possible } \\
\text { to test }\end{array}$ & $\begin{array}{c}\text { Not possible } \\
\text { to test }\end{array}$ \\
\hline $\begin{array}{c}4 \mathrm{kHz} \text { peak } \\
\text { II-III }\end{array}$ & $\begin{array}{l}\text { Control } 0.99 \pm 0.02(n=13) \\
\quad \text { BLZ945 } 1.00 \pm 0.03 \\
(n=14)\end{array}$ & $\begin{array}{l}\text { Control } 0.83 \pm 0.04(n=11) \\
\quad \text { BLZ945 } 0.76 \pm 0.02 \\
(n=15)\end{array}$ & $p=0.7107$ & $p=0.0339$ & $p=0.1573$ & $p=0.0309$ & $p=0.6656$ & $p=0.7258$ & $p=0.0021$ & $p=0.1644$ & $\begin{array}{l}\text { Not possible } \\
\text { to test }\end{array}$ & $p=0.0079$ \\
\hline $\begin{array}{c}8 \mathrm{kHz} \text { peak } \\
\text { II-III }\end{array}$ & $\begin{array}{l}\text { Control } 0.84 \pm 0.01(n=13) \\
\quad \text { BLZ945 } 1.01 \pm 0.03 \\
\quad(n=14)\end{array}$ & $\begin{array}{l}\text { Control } 0.81 \pm 0.02(n=11) \\
\quad \text { BLZ945 } 0.79 \pm 0.03 \\
\quad(n=15)\end{array}$ & $p<0.0001$ & $p=0.6447$ & $p=0.0426$ & $p=0.0143$ & $p=0.6052$ & $p=0.9630$ & $p=0.0179$ & $p=0.8336$ & $\begin{array}{l}\text { Not possible } \\
\text { to test }\end{array}$ & $p=0.9809$ \\
\hline $\begin{array}{r}12 \mathrm{kHz} \\
\text { peak } \\
\text { II-III }\end{array}$ & $\begin{array}{l}\text { Control } 0.89 \pm 0.01(n=13) \\
\quad \text { BLZ945 } 1.04 \pm 0.02 \\
(n=14)\end{array}$ & $\begin{array}{l}\text { Control } 0.83 \pm 0.03(n=11) \\
\quad \text { BLZ945 } 0.85 \pm 0.02 \\
(n=15)\end{array}$ & $p<0.0001$ & $p=0.4802$ & $p=0.1744$ & $p=0.0359$ & $p=0.7669$ & $p=0.8068$ & $p=0.1599$ & $p=0.9206$ & $p<0.0001$ & $p=0.2105$ \\
\hline $\begin{array}{r}16 \mathrm{kHz} \\
\text { peak } \\
\text { II-III }\end{array}$ & $\begin{array}{l}\text { Control } 0.97 \pm 0.03(n=13) \\
\quad \text { BLZ945 } 1.13 \pm 0.01 \\
\quad(n=14)\end{array}$ & $\begin{array}{l}\text { Control } 0.89 \pm 0.03(n=11) \\
\quad \text { BLZ945 } 0.93 \pm 0.02 \\
\quad(n=15)\end{array}$ & $p<0.0001$ & $p=0.1727$ & $p=0.3229$ & $p=0.0254$ & $p=0.8809$ & $p=0.9121$ & $p=0.0957$ & $p=0.8282$ & $p<0.0001$ & $p=0.1373$ \\
\hline $\begin{array}{r}24 \mathrm{kHz} \\
\text { peak } \\
\text { II-III }\end{array}$ & $\begin{array}{l}\text { Control 1.01 } \pm 0.02(n=13) \\
\quad \text { BLZ945 } 1.32 \pm 0.02 \\
\quad(n=14)\end{array}$ & $\begin{array}{l}\text { Control } 1.02 \pm 0.06(n=11) \\
\quad \text { BLZ945 } 1.03 \pm 0.03 \\
\quad(n=15)\end{array}$ & $p<0.0001$ & $p=0.0306$ & $p=0.8895$ & $p=0.7698$ & $p=0.8571$ & $p=0.1234$ & $p=0.8095$ & $p=0.0101$ & $p<0.0001$ & $\begin{array}{c}\text { Not possible } \\
\text { to test }\end{array}$ \\
\hline $\begin{array}{r}32 \mathrm{kHz} \\
\text { peak } \\
\text { II-III }\end{array}$ & $\begin{array}{l}\text { Control } 1.02 \pm 0.02(n=13) \\
\quad \text { BLZ945 } 1.35 \pm 0.02 \\
(n=14)\end{array}$ & $\begin{array}{l}\text { Control } 0.94 \pm 0.03(n=11) \\
\quad \text { BLZ945 } 1.20 \pm 0.05 \\
\quad(n=14)\end{array}$ & $p<0.0001$ & $p<0.0001$ & $p=0.7796$ & $p=0.4237$ & $p=0.7163$ & $p=0.6276$ & $p=0.6767$ & $p=0.0622$ & $p<0.0001$ & $p<0.0001$ \\
\hline
\end{tabular}


Table 4: Continued

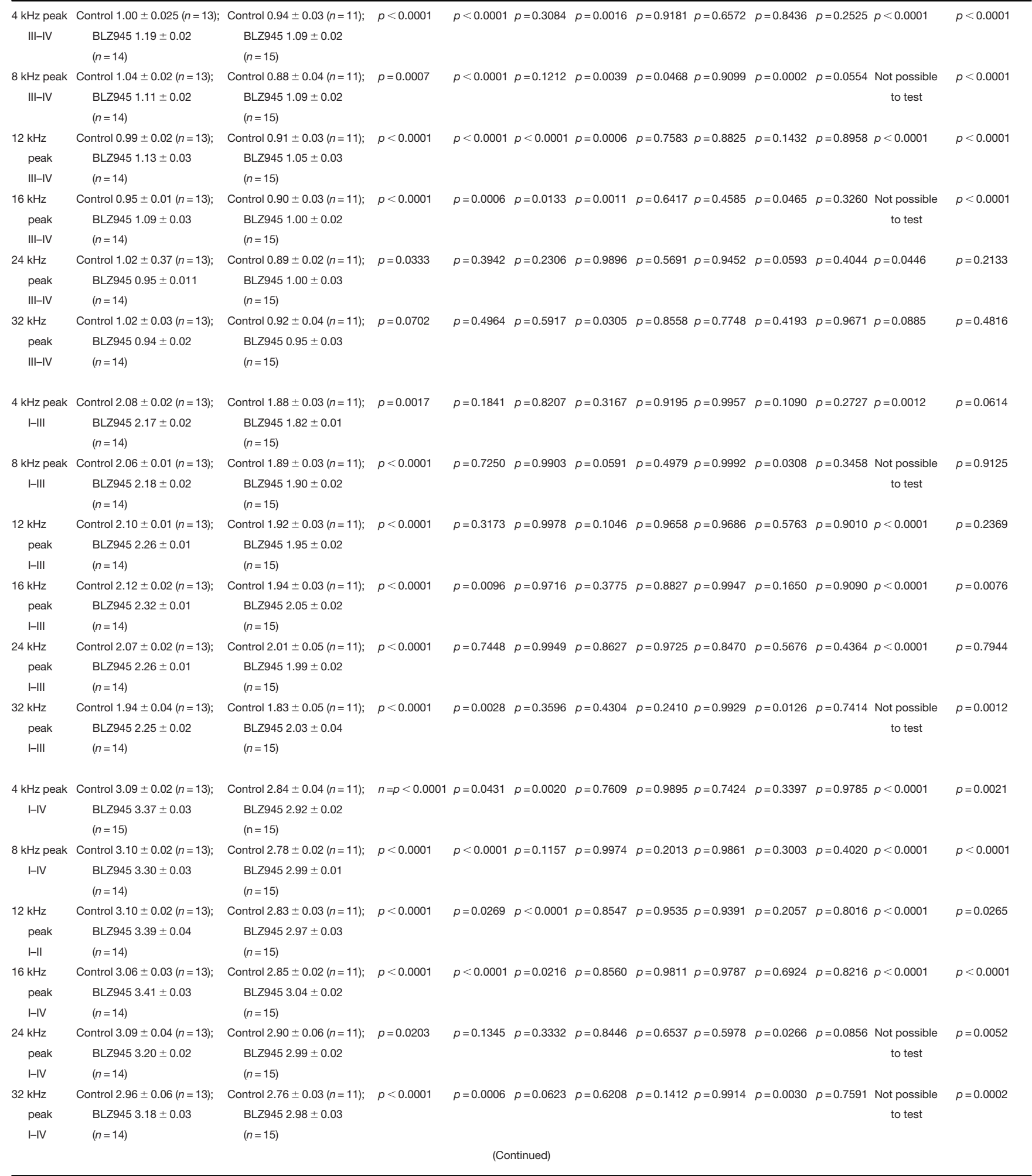


Table 4: Continued

\begin{tabular}{|c|c|c|c|c|c|c|c|c|c|c|c|c|}
\hline \multirow[b]{2}{*}{ Stimulus } & \multirow[b]{2}{*}{4 weeks } & \pm SEM & peak amplituc & $(\mu \mathrm{V})(\mathrm{ms})$ & \multicolumn{2}{|c|}{ Intensity } & \multicolumn{2}{|c|}{ Interaction } & $\begin{array}{l}\text { Simple line } \\
\text { Differ } \\
\text { betweer }\end{array}$ & $\begin{array}{l}\text { earregression } \\
\text { rences } \\
\text { n slopes }\end{array}$ & $\begin{array}{r}\text { Differen } \\
\text { elev } \\
\text { int }\end{array}$ & $\begin{array}{l}\text { es between } \\
\text { tions or } \\
\text { cepts }\end{array}$ \\
\hline & & 7 weeks & 4 weeks & 7 weeks & 4 weeks & 7 weeks & 4 weeks & 7 weeks & 4 weeks & 7 weeks & 4 weeks & 7 weeks \\
\hline $\begin{array}{l}4 \mathrm{kHz} \\
\quad \text { peak I }\end{array}$ & $\begin{array}{l}\text { Control 2.44 } \pm 0.33(n=13) ; \\
\quad \text { BLZ945 } 2.19 \pm 0.43 \\
(n=14)\end{array}$ & $\begin{array}{l}\text { Control 1.18 } \pm 0.13(n=11) \\
\quad \text { BLZ945 } 1.04 \pm 0.14 \\
(n=15)\end{array}$ & $p=0.0321$ & $p=0.2298$ & $p<0.0001$ & $p<0.0001$ & $p=0.4344$ & $p>0.9999$ & $p=0.0003$ & $p=0.7428$ & $\begin{array}{c}\text { Not possible } \\
\text { to test }\end{array}$ & $p=0.1463$ \\
\hline $\begin{array}{l}8 \mathrm{kHz} \\
\quad \text { peak I }\end{array}$ & $\begin{array}{l}\text { Control } 2.35 \pm 0.40(n=13) \\
\quad \text { BLZ945 } 2.14 \pm 0.40 \\
(n=14)\end{array}$ & $\begin{array}{l}\text { Control } 1.05 \pm 0.14(n=11) \\
\quad \text { BLZ945 } 0.94 \pm 0.14 \\
(n=15)\end{array}$ & $p<0.0001$ & $p=0.2580$ & $p<0.0001$ & $p<0.0001$ & $p=0.9867$ & $p=0.9896$ & $p=0.6324$ & $p=0.5651$ & $p=0.0219$ & $p=0.1100$ \\
\hline $\begin{array}{l}12 \mathrm{kHz} \\
\text { peak I }\end{array}$ & $\begin{array}{l}\text { Control 2.28 } \pm 0.40(n=13) \\
\quad \text { BLZ945 } 1.78 \pm 0.35 \\
(n=14)\end{array}$ & $\begin{array}{l}\text { Control 1.01 } \pm 0.12(n=11) \\
\quad \text { BLZ945 } 0.96 \pm 0.15 \\
(n=15)\end{array}$ & $p<0.0001$ & $p=0.5704$ & $p<0.0001$ & $p<0.0001$ & $p=0.9867$ & $p=0.9970$ & $p=0.6766$ & $p=0.2858$ & $p<0.0001$ & $p=0.6782$ \\
\hline $\begin{array}{l}16 \mathrm{kHz} \\
\text { peak I }\end{array}$ & $\begin{array}{l}\text { Control 2.12 } \pm 0.36(n=13) \\
\quad \text { BLZ945 } 1.80 \pm 0.33 \\
(n=14)\end{array}$ & $\begin{array}{l}\text { Control } 0.95 \pm 0.14(n=11) \\
\quad \text { BLZ945 } 0.91 \pm 0.12 \\
(n=15)\end{array}$ & $p=0.0002$ & $p=0.6791$ & $p<0.0001$ & $p<0.0001$ & $p=0.9739$ & $p=0.9987$ & $p=0.4497$ & $p=0.3577$ & $p<0.0001$ & $p=0.4971$ \\
\hline $\begin{array}{l}24 \mathrm{kHz} \\
\text { peak I }\end{array}$ & $\begin{array}{l}\text { Control 1.57 } \pm 0.32(n=13) \\
\quad \text { BLZ945 } 1.40 \pm 0.28 \\
(n=14)\end{array}$ & $\begin{array}{l}\text { Control } 0.80 \pm 0.13(n=11) \\
\quad \text { BLZ945 } 0.75 \pm 0.11 \\
(n=15)\end{array}$ & $p=0.0220$ & $p=0.5020$ & $p<0.0001$ & $p<0.0001$ & $p=0.8737$ & $p=0.9967$ & $p=0.0933$ & $p=0.6140$ & $p=0.0164$ & $p=0.3243$ \\
\hline $\begin{array}{l}32 \mathrm{kHz} \\
\text { peak I }\end{array}$ & $\begin{array}{l}\text { Control 1.22 } \pm 0.26(n=13) \\
\quad \text { BLZ945 1.21 } \pm 0.23 \\
(n=14)\end{array}$ & $\begin{array}{l}\text { Control } 0.42 \pm 0.09(n=11) \\
\quad \text { BLZ945 } 0.77 \pm 0.09 \\
(n=15)\end{array}$ & $p=0.9183$ & $p=0.7245$ & $p<0.0001$ & $p<0.0001$ & $p=0.8965$ & $p=0.9998$ & $p=0.4540$ & $p=0.9961$ & $p=0.6648$ & $p=0.6956$ \\
\hline $\begin{array}{l}4 \mathrm{kHz} \\
\quad \text { peak II }\end{array}$ & $\begin{array}{l}\text { Control } 3.18 \pm 0.26(n=13) \\
\quad \text { BLZ945 } 2.89 \pm 0.54 \\
(n=14)\end{array}$ & $\begin{array}{l}\text { Control } 2.34 \pm 0.25(n=11) \\
\quad \text { BLZ945 } 2.31 \pm 0.18 \\
(n=15)\end{array}$ & $p=0.1470$ & $p=0.8947$ & $p<0.0001$ & $p<0.0001$ & $p=0.0065$ & $p=0.8911$ & $p<0.0001$ & $p=0.1516$ & $\begin{array}{c}\text { Not possible } \\
\text { to test }\end{array}$ & $p=0.3248$ \\
\hline $\begin{array}{l}8 \mathrm{kHz} \\
\quad \text { peak II }\end{array}$ & $\begin{array}{l}\text { Control } 3.79 \pm 0.43(n=13) \\
\quad \text { BLZ945 } 2.80 \pm 0.49 \\
(n=14)\end{array}$ & $\begin{array}{l}\text { Control } 2.44 \pm 0.30(n=11) \\
\quad \text { BLZ945 } 2.19 \pm 0.21 \\
(n=15)\end{array}$ & $p<0.0001$ & $p=0.1876$ & $p<0.0001$ & $p<0.0001$ & $p=0.5675$ & $p=0.9756$ & $p=0.9709$ & $p=0.1704$ & $p<0.0001$ & $p=0.0174$ \\
\hline $\begin{array}{l}12 \mathrm{kHz} \\
\text { peak II }\end{array}$ & $\begin{array}{l}\text { Control } 3.68 \pm 0.42(n=13) \\
\quad \text { BLZ945 } 2.51 \pm 0.47 \\
(n=14)\end{array}$ & $\begin{array}{l}\text { Control 2.46 } \pm 0.29(n=11) \\
\quad \text { BLZ945 } 2.25 \pm 0.29 \\
(n=15)\end{array}$ & $p<0.0001$ & $p=0.2617$ & $p<0.0001$ & $p<0.0001$ & $p=0.0448$ & $p=0.9824$ & $p=0.0264$ & $p=0.9547$ & & $p=0.0847$ \\
\hline $\begin{array}{l}16 \mathrm{kHz} \\
\text { peak II }\end{array}$ & $\begin{array}{l}\text { Control } 3.57 \pm 0.43(n=13) ; \\
\quad \text { BLZ945 } 2.59 \pm 0.42 \\
(n=14)\end{array}$ & $\begin{array}{l}\text { Control } 2.44 \pm 0.32(n=11) \\
\quad \text { BLZ945 } 2.28 \pm 0.21 \\
(n=15)\end{array}$ & $p<0.0001$ & $p=0.3469$ & $p<0.0001$ & $p<0.0001$ & $p=0.9849$ & $p=0.6964$ & $p=0.4983$ & $p=0.0200$ & $p<0.0001$ & $\begin{array}{c}\text { Not possible } \\
\text { to test }\end{array}$ \\
\hline $\begin{array}{l}24 \mathrm{kHz} \\
\text { peak II }\end{array}$ & $\begin{array}{l}\text { Control 1.90 } \pm 0.18(n=13) ; \\
\quad \text { BLZ945 } 1.61 \pm 0.11 \\
(n=14)\end{array}$ & $\begin{array}{l}\text { Control 1.31 } \pm 0.15(n=11) \\
\quad \text { BLZ945 } 1.22 \pm 0.03 \\
\quad(n=15)\end{array}$ & $p=0.0053$ & $p=0.4828$ & $p<0.0001$ & $p=0.2868$ & $p=0.1501$ & $p=0.2803$ & $p=0.0097$ & $p=0.0010$ & $\begin{array}{c}\text { Not possible } \\
\text { to test }\end{array}$ & $\begin{array}{c}\text { Not possible } \\
\text { to test }\end{array}$ \\
\hline $\begin{array}{l}32 \mathrm{kHz} \\
\text { peak II }\end{array}$ & $\begin{array}{l}\text { Control 1.41 } \pm 0.10(n=13) ; \\
\quad \text { BLZ945 1.30 } \pm 0.10 \\
(n=14)\end{array}$ & $\begin{array}{l}\text { Control 1.23 } \pm 0.09(n=11) \\
\quad \text { BLZ945 1.18 } \\
\quad(n=15)\end{array}$ & $p=0.5935$ & $p=0.5935$ & $p=0.0048$ & $p=0.8206$ & $p=0.6878$ & $p=0.5643$ & $p=0.0503$ & $p=0.0184$ & $p=0.4283$ & $\begin{array}{c}\text { Not possible } \\
\text { to test }\end{array}$ \\
\hline $\begin{array}{l}4 \mathrm{kHz} \\
\quad \text { peak III }\end{array}$ & $\begin{array}{l}\text { Control } 2.71 \pm 0.36(n=13) \\
\quad \text { BLZ945 } 1.83 \pm 0.30 \\
(n=14)\end{array}$ & $\begin{array}{l}\text { Control } 1.54 \pm 0.25(n=11) \\
\quad \text { BLZ945 } 1.60 \pm 0.23 \\
(n=15)\end{array}$ & $p<0.0001$ & $p=0.5893$ & $p<0.0001$ & $p<0.0001$ & $p=0.9414$ & $p=0.6150$ & $p=0.4075$ & $p=0.9775$ & $p<0.0001$ & $p=0.9607$ \\
\hline $\begin{array}{l}8 \mathrm{kHz} \\
\quad \text { peak III }\end{array}$ & $\begin{array}{l}\text { Control 2.87 } \pm 0.40(n=13) \\
\quad \text { BLZ945 } 2.16 \pm 0.33 \\
(n=14)\end{array}$ & $\begin{array}{l}\text { Control 1.96 } \pm 0.32(n=11) \\
\quad \text { BLZ945 } 1.87 \pm 0.25 \\
\quad(n=15)\end{array}$ & $p<0.0001$ & $p=0.4410$ & $p<0.0001$ & $p<0.0001$ & $p=0.9013$ & $p=0.6888$ & $p=0.3396$ & $p=0.0894$ & $p<0.0001$ & $p=0.0641$ \\
\hline $\begin{array}{l}12 \mathrm{kHz} \\
\quad \text { peak III }\end{array}$ & $\begin{array}{l}\text { Control 2.46 } \pm 0.39(n=13) \\
\quad \text { BLZ945 } 1.75 \pm 0.34 \\
\quad(n=14)\end{array}$ & $\begin{array}{l}\text { Control } 1.70 \pm 0.29(n=11) \\
\quad \text { BLZ945 } 1.65 \pm 0.23 \\
(n=15)\end{array}$ & $p<0.0001$ & $p=0.6165$ & $p<0.0001$ & $p<0.0001$ & $p=0.0003$ & $p=0.7180$ & $p=0.7803$ & $p=0.1604$ & $p<0.0001$ & $p=0.1493$ \\
\hline $\begin{array}{l}16 \mathrm{kHz} \\
\quad \text { peak III }\end{array}$ & $\begin{array}{l}\text { Control } 2.37 \pm 0.38(n=13) ; \\
\quad \text { BLZ945 1.71 } \pm 0.29 \\
(n=14)\end{array}$ & $\begin{array}{l}\text { Control 1.72 } \pm 0.26(n=11) \\
\quad \text { BLZ945 } 1.68 \pm 0.22 \\
\quad(n=15)\end{array}$ & $p<0.0001$ & $p=0.7426$ & $p<0.0001$ & $p<0.0001$ & $p=0.0152$ & $p=0.8593$ & $p=0.0539$ & $p=0.2221$ & $p<0.0001$ & $p=0.5628$ \\
\hline $\begin{array}{l}24 \mathrm{kHz} \\
\quad \text { peak III }\end{array}$ & $\begin{array}{l}\text { Control } 2.19 \pm 0.28(n=13) \\
\quad \text { BLZ945 } 2.07 \pm 0.30 \\
(n=14)\end{array}$ & $\begin{array}{l}\text { Control } 1.80 \pm 0.17(n=11) \\
\quad \text { BLZ945 } 1.77 \pm 0.22 \\
(n=15)\end{array}$ & $p=0.2898$ & $p=0.8442$ & $p<0.0001$ & $p<0.0001$ & $p=0.9822$ & $p=0.8618$ & $p=0.6517$ & $p=0.1920$ & $p=0.2912$ & $p=0.8913$ \\
\hline $\begin{array}{l}32 \mathrm{kHz} \\
\quad \text { peak III }\end{array}$ & $\begin{array}{l}\text { Control 1.89 } \pm 0.28(n=13) ; \\
\quad \text { BLZ945 } 2.07 \pm 0.27 \\
(n=14)\end{array}$ & $\begin{array}{l}\text { Control } 1.45 \pm 0.16(n=11) \\
\quad \text { BLZ945 } 1.74 \pm 0.15 \\
(n=15)\end{array}$ & $p=0.0895$ & $p=0.0329$ & $p<0.0001$ & $p<0.0001$ & $p=0.9441$ & $p=0.9858$ & $p=0.9026$ & $p=0.8422$ & $p=0.0880$ & $p=0.0143$ \\
\hline
\end{tabular}


Table 4: Continued

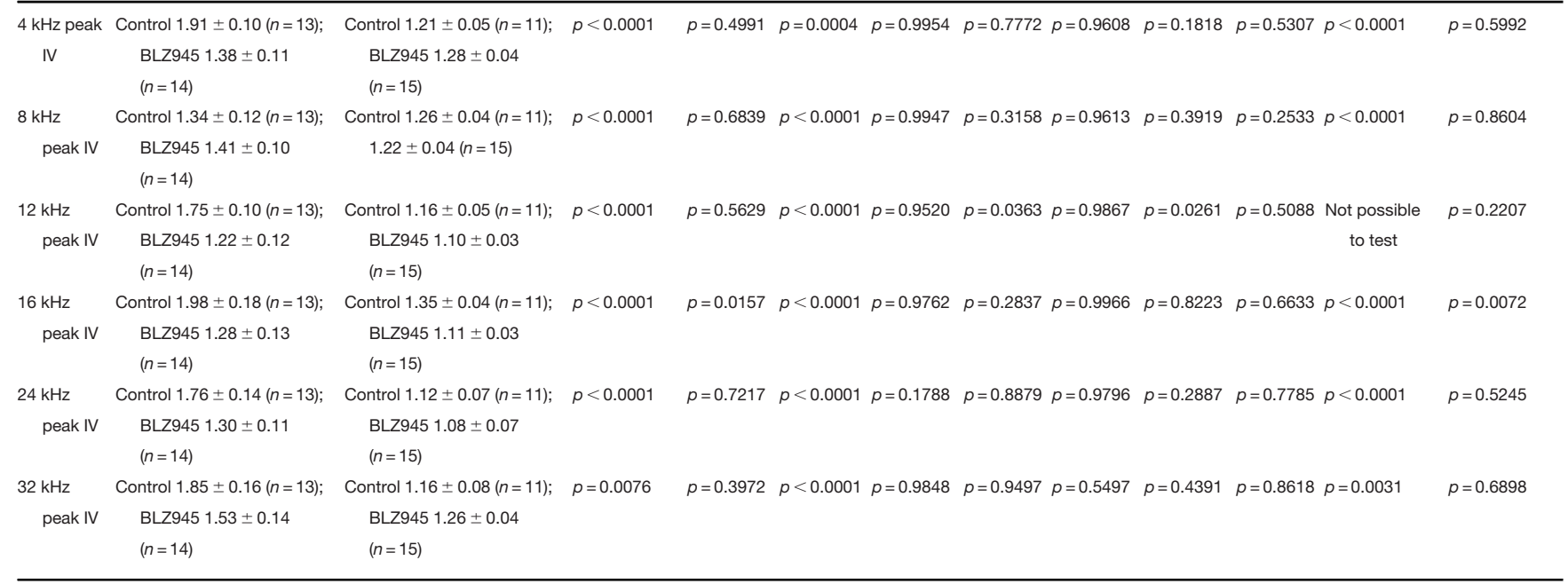

show any intensity-specific differences (Tables 4, 5), suggesting an overall decrease in the effect of the treatment. In peak IV (Fig. 5D), BLZ945 treatment resulted in significantly increased latencies at four weeks in the lower and middle frequencies, but not the high frequencies. At seven weeks, latencies showed some recovery in the middle frequencies, but BL7945-treated mice still showed significantly increased latencies at $32 \mathrm{kHz}$ (Fig. 5D). Together, these results indicate that temporary CSF1R inhibition early in development affects peak latencies at four weeks. Some, but not all, of these latency changes recover after cessation of drug treatment by seven weeks.

\section{Increased interpeak latencies partially recover by seven weeks of age}

We next measured interpeak latencies to test whether the observed latency differences in the BLZ945-treated mice is because of peripheral conduction defects, reflect any delays in central activity or is a result of both. Interpeak latency was determined as the difference in time between the apex of the peak and the apex of the preceding peak (ms). At four weeks, treatment effects on interpeak latencies were observed between peaks I and II at 8 and $24 \mathrm{kHz}$, where BLZ945-treated mice showed significantly decreased interpeak latencies. However, by seven weeks, there were no differences in peak I-II interpeak latencies, indicating that cochlea-SGN-VIIIth nerve signal transduction recovered (Fig. 6A). BLZ945 treatment led to significantly increased peak II-III interpeak latencies at four weeks at $8,12,16,24$, and $32 \mathrm{kHz}$ (Fig. 6B). At seven weeks, BLZ945-treated mice showed normal peak II-III latencies at the lower and middle frequencies, but the higher frequencies remained delayed (Fig. 6B). Together, these data suggest that signal transmission from the $\mathrm{CN}$ to the SOC is delayed after temporary microglia depletion but recovers by at seven weeks. Peak III-IV interpeak latencies (Fig. 6C) were significantly elevated at 4, 8, 12, 16, and $24 \mathrm{kHz}$ in BLZ945-treated mice at four weeks. Post hoc multiple comparison analyses showed that these interpeak latency differences were more apparent in the lower frequencies. Similar results were obtained at seven weeks, indicating that these increases in peak III-IV latencies do not recover with time (Fig. 6C).

To investigate the overall effects of temporary CSF1R inhibition on central latencies, we measured interpeak latencies between peaks I-III and I-IV. BLZ945 treatment led to increased interpeak latencies between peaks I and III at four weeks at all frequencies tested when compared with control mice. At seven weeks, peak I-III latencies improved at most frequency levels except for 16 and $32 \mathrm{kHz}$ (Tables 4, 5). Treatment effect comparisons for peak I-IV latencies showed a significant increase in the BLZ945treated group at all frequencies tested at both four and seven weeks compared with controls (Fig. 6D). However, post hoc comparisons did not display any significant changes for any particular intensity at seven weeks, indicating some recovery in central latencies after prolonged microglia repopulation. Together, these findings demonstrate that BLZ945 treatment in the early postnatal period increases latencies in the auditory brainstem measured at four weeks, and that these effects are partially improved by seven weeks.

\section{Peak amplitudes are diminished at four weeks but recover by seven weeks of age}

We next examined the effect of BLZ945 treatment on ABR peak amplitudes, determined as the difference in $\mu \mathrm{V}$ from the preceding trough to the following peak. Peak I (Fig. 7A) amplitude showed that there was a significant decrease in treated animals across all frequencies except 8 and $32 \mathrm{kHz}$ at four weeks. Post hoc multiple comparisons tests did not show significant differences at any specific intensity at all frequency levels. At seven weeks, peak I amplitude did not differ between BLZ945-treated mice and control mice. Peak II amplitude (Fig. 7B) was significantly diminished at $8,12,16$, and $24 \mathrm{kHz}$ at most intensities in BLZ945-treated mice at four weeks. By seven weeks, peak II amplitudes were comparable to control levels at all frequencies and intensities (Fig. 7B). BLZ945 treatment at four weeks resulted in diminished 
Table 5: Sidak's multiple comparisons

\begin{tabular}{|c|c|c|c|c|c|c|c|c|c|c|c|c|c|}
\hline \multirow[b]{3}{*}{ Peak I } & \multirow[b]{3}{*}{ Frequency $(\mathrm{kHz})$} & \multicolumn{12}{|c|}{ Absolute peak latency (ms) } \\
\hline & & \multicolumn{2}{|c|}{4} & \multicolumn{2}{|c|}{8} & \multicolumn{2}{|c|}{12} & \multicolumn{2}{|c|}{16} & \multicolumn{2}{|c|}{24} & \multicolumn{2}{|c|}{32} \\
\hline & & 4 weeks & 7 weeks & 4 weeks & 7 weeks & 4 weeks & 7 weeks & 4 weeks & 7 weeks & 4 weeks & 7 weeks & 4 weeks & 7 weeks \\
\hline \multirow[t]{15}{*}{ Intensity (dB SPL) } & 80 & $<0.0001$ & 0.0154 & 0.142 & 0.0704 & 0.9144 & 0.073 & 0.0148 & 0.9989 & 0.971 & $>0.9999$ & 0.9719 & $>0.9999$ \\
\hline & 75 & $<0.0001$ & 0.0161 & 0.0107 & 0.6141 & 0.3324 & 0.8812 & 0.0164 & 0.3791 & 0.9382 & $>0.9999$ & 0.8797 & $>0.9999$ \\
\hline & 70 & 0.0002 & 0.0035 & 0.0063 & 0.1509 & 0.3323 & 0.6701 & 0.7302 & 0.5615 & 0.6526 & $>0.9999$ & 0.9902 & $>0.9999$ \\
\hline & 65 & 0.0005 & 0.0002 & 0.004 & 0.015 & 0.0062 & 0.3586 & 0.7599 & 0.7856 & 0.8392 & 0.9998 & 0.9781 & $>0.9999$ \\
\hline & 60 & $<0.0001$ & 0.0001 & 0.0819 & 0.041 & 0.1472 & 0.3005 & $>0.9999$ & 0.3754 & 0.0982 & 0.996 & 0.8802 & $>0.9999$ \\
\hline & 55 & $<0.0001$ & 0.0002 & 0.1598 & 0.0687 & 0.4281 & 0.2698 & $>0.9999$ & 0.6131 & 0.5943 & $>0.9999$ & 0.9438 & 0.9891 \\
\hline & 50 & $<0.0001$ & 0.0003 & 0.0517 & 0.076 & 0.9968 & 0.3716 & $>0.9999$ & 0.2265 & 0.4666 & 0.9915 & 0.6895 & $>0.9999$ \\
\hline & 45 & $<0.0001$ & 0.0003 & 0.0001 & 0.0235 & 0.9997 & 0.9985 & $>0.9999$ & 0.1687 & 0.1566 & $>0.9999$ & 0.9761 & $>0.9999$ \\
\hline & 40 & $<0.0001$ & 0.0569 & 0.0004 & 0.3129 & $>0.9999$ & 0.9915 & $>0.9999$ & 0.616 & 0.0441 & 0.9885 & 0.9999 & 0.9919 \\
\hline & 35 & $<0.0001$ & 0.6835 & 0.0591 & $>0.9999$ & 0.5309 & $>0.9999$ & $>0.9999$ & 0.8837 & & 0.9894 & & \\
\hline & 30 & 0.0027 & $>0.9999$ & & $>0.9999$ & 0.2878 & $>0.9999$ & 0.9738 & 0.6585 & & & & \\
\hline & 25 & & 0.9999 & & $>0.9999$ & 0.739 & $>0.9999$ & $>0.9999$ & 0.9991 & & & & \\
\hline & 20 & & 0.995 & & $>0.9999$ & $>0.9999$ & $>0.9999$ & & & & & & \\
\hline & 15 & & 0.9948 & & & & 0.9977 & & & & & & \\
\hline & 10 & & & & & & & & & & & & \\
\hline \multirow[t]{2}{*}{ Peak II } & Frequency $(\mathrm{kHz})$ & \multicolumn{2}{|c|}{4} & & & & & & & & & & \\
\hline & & 4 weeks & 7 weeks & 4 weeks & 7 weeks & 4 weeks & 7 weeks & 4 weeks & 7 weeks & 4 weeks & 7 weeks & 4 weeks & 7 weeks \\
\hline Intensity (dB SPL) & 80 & 0.015 & 0.5411 & 0.9932 & 0.9179 & 0.9844 & 0.9554 & 0.7955 & 0.9297 & $>0.9999$ & 0.9601 & $>0.9999$ & 0.9604 \\
\hline & 75 & 0.0829 & 0.623 & 0.9826 & 0.9494 & 0.8154 & 0.9389 & 0.8754 & 0.9598 & 0.7029 & 0.7113 & 0.9999 & 0.9616 \\
\hline & 70 & 0.0629 & 0.8672 & 0.9968 & 0.9987 & 0.1797 & 0.9887 & 0.9518 & 0.9694 & 0.2931 & 0.7088 & 0.9784 & 0.6067 \\
\hline & 65 & 0.3147 & 0.4749 & 0.9956 & $>0.9999$ & 0.6802 & 0.9929 & 0.8351 & 0.8741 & 0.1661 & 0.802 & 0.9994 & 0.93 \\
\hline & 60 & 0.7408 & 0.0855 & $>0.9999$ & 0.9996 & 0.3242 & $>0.9999$ & 0.9547 & 0.9938 & 0.1172 & 0.9022 & 0.9941 & 0.9996 \\
\hline & 55 & 0.9414 & 0.0574 & 0.9749 & 0.999 & $>0.9999$ & 0.9772 & 0.9981 & 0.6225 & 0.1616 & 0.9837 & 0.9287 & 0.9998 \\
\hline & 50 & 0.885 & 0.6198 & $>0.9999$ & 0.8977 & $>0.9999$ & $>0.9999$ & $>0.9999$ & 0.9004 & 0.8599 & 0.9996 & 0.7632 & $>0.9999$ \\
\hline & 45 & 0.0736 & 0.7625 & 0.8146 & 0.9982 & $>0.9999$ & $>0.9999$ & $>0.9999$ & 0.9991 & 0.6202 & $>0.9999$ & 0.4334 & 0.9974 \\
\hline & 40 & 0.1948 & $>0.9999$ & 0.7421 & $>0.9999$ & $>0.9999$ & 0.9819 & $>0.9999$ & $>0.9999$ & 0.4782 & 0.9989 & 0.0707 & 0.9294 \\
\hline & 35 & 0.3042 & 0.9998 & 0.5336 & 0.998 & $>0.9999$ & 0.9998 & 0.9978 & 0.9612 & 0.3795 & 0.9992 & & \\
\hline & 30 & 0.2715 & $>0.9999$ & & $>0.9999$ & $>0.9999$ & $>0.9999$ & $>0.9999$ & 0.814 & & & & \\
\hline & 25 & & $>0.9999$ & & 0.9958 & 0.9984 & $>0.9999$ & 0.9715 & 0.9212 & & & & \\
\hline & 20 & & 0.9924 & & 0.9633 & 0.7481 & 0.9998 & & & & & & \\
\hline & 15 & & 0.997 & & & & & & & & & & \\
\hline & 10 & & & & & & & & & & & & \\
\hline Peak III & Frequency (kHz) & & & & & & & & & & & & \\
\hline & & 4 weeks & 7 weeks & 4 weeks & 7 weeks & 4 weeks & 7 weeks & 4 weeks & 7 weeks & 4 weeks & 7 weeks & 4 weeks & 7 weeks \\
\hline Intensity (dB SPL) & 80 & 0.0892 & $>0.9999$ & 0.2589 & 0.9997 & 0.6618 & 0.9508 & 0.0869 & 0.6097 & 0.7121 & $>0.9999$ & 0.0326 & 0.9975 \\
\hline & 75 & 0.0931 & $>0.9999$ & 0.7414 & 0.9999 & 0.0564 & 0.9542 & 0.0339 & 0.972 & 0.9859 & $>0.9999$ & 0.4689 & 0.825 \\
\hline & 70 & 0.1659 & $>0.9999$ & 0.1266 & $>0.9999$ & 0.0024 & 0.9981 & 0.0523 & 0.9129 & $>0.9999$ & $>0.9999$ & 0.3291 & 0.9981 \\
\hline & 65 & 0.2881 & 0.9999 & 0.1423 & 0.9998 & 0.0101 & $>0.9999$ & 0.0934 & 0.9992 & $>0.9999$ & 0.9997 & 0.5173 & 0.989 \\
\hline & 60 & 0.5546 & 0.9817 & 0.4306 & 0.9383 & 0.3956 & $>0.9999$ & 0.2073 & 0.9989 & $>0.9999$ & 0.9995 & 0.9985 & 0.9686 \\
\hline & 55 & 0.3345 & 0.922 & 0.3093 & 0.9867 & 0.5178 & 0.8474 & 0.3623 & 0.9216 & $>0.9999$ & $>0.9999$ & $>0.9999$ & 0.9907 \\
\hline & 50 & 0.1898 & 0.9493 & 0.1087 & 0.9832 & 0.6383 & 0.7323 & 0.7939 & 0.9927 & $>0.9999$ & $>0.9999$ & 0.9995 & 0.9991 \\
\hline & 45 & 0.0022 & 0.9873 & 0.008 & $>0.9999$ & 0.6042 & 0.9886 & 0.8632 & 0.6164 & $>0.9999$ & 0.9565 & 0.9992 & 0.9993 \\
\hline & 40 & 0.0015 & 0.9906 & 0.0013 & 0.988 & 0.2169 & 0.9999 & 0.9932 & 0.8396 & $>0.9999$ & 0.9997 & $>0.9999$ & 0.9031 \\
\hline & 35 & 0.0324 & $>0.9999$ & 0.0005 & $>0.9999$ & 0.3112 & $>0.9999$ & 0.1275 & 0.9472 & & $>0.9999$ & & \\
\hline & 30 & 0.113 & $>0.9999$ & & $>0.9999$ & 0.095 & $>0.9999$ & $>0.9999$ & 0.9577 & & & & \\
\hline & 25 & & $>0.9999$ & & $>0.9999$ & 0.0471 & $>0.9999$ & $>0.9999$ & 0.9943 & & & & \\
\hline & 20 & & 0.9954 & & 0.9998 & 0.7571 & 0.9989 & & & & & & \\
\hline & 15 & & $>0.9999$ & & & & 0.9998 & & & & & & \\
\hline & 10 & & & & & & & & & & & & \\
\hline Peak IV & Frequency (kHz) & & & & 3 & & & & & & & & \\
\hline & & 4 weeks & 7 weeks & 4 weeks & 7 weeks & 4 weeks & 7 weeks & 4 weeks & 7 weeks & 4 weeks & 7 weeks & 4 weeks & 7 weeks \\
\hline Intensity (dB SPL) & 80 & 0.002 & 0.9658 & 0.0093 & 0.4017 & 0.0385 & 0.4587 & 0.0096 & 0.4029 & 0.8486 & 0.8909 & 0.6344 & 0.9758 \\
\hline & 75 & 0.0007 & 0.8294 & 0.0146 & 0.0402 & 0.0456 & 0.7979 & 0.0106 & 0.7975 & 0.9672 & 0.946 & 0.98 & 0.8348 \\
\hline & 70 & 0.0014 & 0.3602 & 0.0013 & 0.0924 & 0.0135 & 0.6328 & 0.0274 & 0.1067 & $>0.9999$ & 0.9875 & 0.4351 & 0.976 \\
\hline & 65 & 0.0022 & 0.2856 & 0.0025 & 0.079 & 0.0131 & 0.8566 & 0.0554 & 0.9267 & $>0.9999$ & 0.9856 & 0.4067 & 0.9011 \\
\hline & 60 & 0.0014 & 0.0079 & 0.0901 & 0.0881 & 0.0488 & 0.9458 & 0.0369 & 0.8932 & $>0.9999$ & 0.3691 & $>0.9999$ & $>0.9999$ \\
\hline & 55 & 0.0006 & 0.2969 & 0.8889 & 0.1695 & $\begin{array}{c}0.0777 \\
\text { ontinued) }\end{array}$ & 0.1033 & 0.0248 & 0.4111 & $>0.9999$ & 0.9782 & $>0.9999$ & 0.9987 \\
\hline
\end{tabular}


Table 5: Continued

Absolute peak latency $(\mathrm{ms})$

1216

24

32

$\underline{P e a k ~ I ~}$

\begin{tabular}{|c|c|c|c|c|c|c|c|c|c|c|c|}
\hline 4 weeks & 7 weeks & 4 weeks & 7 weeks & 4 weeks & 7 weeks & 4 weeks & 7 weeks & 4 weeks & 7 weeks & 4 weeks & 7 weeks \\
\hline$<0.0001$ & 0.1709 & 0.4232 & 0.0626 & 0.0244 & 0.2693 & 0.4096 & 0.179 & 0.9968 & 0.9961 & $>0.9999$ & 0.9997 \\
\hline$<0.0001$ & 0.0244 & 0.115 & 0.4409 & 0.1032 & 0.6082 & 0.0112 & 0.4635 & 0.8842 & 0.9997 & 0.8844 & 0.9483 \\
\hline 0.0004 & 0.5158 & 0.0035 & 0.2362 & 0.0416 & 0.9972 & 0.2211 & 0.239 & 0.9608 & $>0.9999$ & 0.8327 & 0.8375 \\
\hline \multirow[t]{5}{*}{0.0087} & $>0.9999$ & 0.0073 & $>0.9999$ & 0.0621 & 0.9932 & 0.0177 & 0.9993 & & 0.9996 & 0.9989 & \\
\hline & $>0.9999$ & & 0.9999 & 0.0005 & $>0.9999$ & 0.7953 & 0.9605 & & & & \\
\hline & 0.9782 & & 0.9988 & 0.0238 & $>0.9999$ & & 0.9844 & & & & \\
\hline & 0.9951 & & 0.9606 & & 0.9164 & & & & & & \\
\hline & $>0.9999$ & & & & 0.3562 & & & & & & \\
\hline
\end{tabular}

$\begin{array}{ll}\text { Peak I-II } & \text { Frequency }(\mathrm{kHz}) \\ \text { Intensity (dB SPL) } & 80 \\ & 75 \\ & 70 \\ 65 \\ 60 \\ 55 \\ 50 \\ 45 \\ 40 \\ 35 \\ 30 \\ 25 \\ 20 \\ 15 \\ 10\end{array}$

4

$0.5664>0.9999>0.9999>0.9999$

$0.747>0.9999 \quad 0.9902$

$0.9919>0.9999 \quad 0.9878$

$>0.9999>0.9999 \quad 0.6939$

$\begin{array}{lll}>0.9999 & 0.9982 & 0.19\end{array}$

$>0.9999>0.9999 \quad 0.8245$

$0.997>0.9999 \quad 0.9989$

$\begin{array}{lll}0.9996 & 0.9537 & >0.9999\end{array}$

0.9997

0.9546

$>0.9999 \quad 0.9998$

$>0.9999$

$>0.9999$

$>0.9999$

$>0.9999$

Peak II-III Frequency $(\mathrm{kHz})$

Intensity (dB SPL) 80

$$
\begin{aligned}
& 80 \\
& 75 \\
& 70
\end{aligned}
$$

70
65

65
60

55

50

45
40

40
35

35
30

25

20

15

10

Peak III-IV

Frequency (kHz)

Intensity (dB SPL) 80

75
70

70
65

65

55

50

45

40

35
30
4

4 weeks 7 weeks

$0.8707 \quad 0.6113$

$0.9998 \quad 0.9919$

$>0.9999 \quad 0.9876$

$>0.9999 \quad 0.9487$

$0.997 \quad 0.9829$

$0.991>0.9999$

$0.9994>0.9999$

$\begin{array}{lll}0.8853 & 0.9619 & 0.0487\end{array}$

$>0.9999>0.9999$

$>0.9999$

0.3171

$>0.9999$

$>0.9999$

4

4 weeks 7 weeks

$0.4531 \quad 0.7043$

$0.2013 \quad 0.8772$

$0.1862 \quad 0.1238$

$0.1297 \quad 0.2098$

$0.0218 \quad 0.0046$

$0.0265 \quad 0.8982$

$0.0075 \quad 0.5608$

$0.1125 \quad 0.0212$

0.9884

0.9805

0.9982

0.3577

0.4207

0.1327

0.0053

0.0375

0.1169

0.759

0.9981
$0.9836 \quad 0.998$

$\begin{array}{lll}0.9999 & >0.9999 \quad 0.0497\end{array}$
0.8972

$>0.9999$
8

12

1682

$>0.9999$

$>0.9999$

$>0.9999$

7 weeks 7 weeks

0.0368

0.0645

$>0.9999 \quad 0.1143$

$>0.9999 \quad 0.3948$

$0.9933 \quad 0.4903$

$>0.9999>0.9999$

$>0.99$

0.6116

0.6543

0.1651

0.6763

0.8028

0.7441

0.02
Interpeak Latency (ms)

7 weeks 4 weeks 7 weeks

24

7 weeks

32

7 weeks

$\begin{array}{lllll}0.977 & 0.7201 & 0.462 & 0.4269\end{array}$

$\begin{array}{lll}>0.9999 & >0.9999 & 0.3337\end{array}$

0.2952

$0.1715>0.9999 \quad 0.0423$

$0.214>0.9999 \quad 0.2511$

$\begin{array}{lll}0.2568 & 0.9999 & 0.9934\end{array}$

$0.9301>0.9999>0.9999$

$0.8362>0.9999>0.9999$

$\begin{array}{lll}>0.9999 & 0.6801 & 0.9948\end{array}$

$>0.9999 \quad 0.8977 \quad 0.9455$

$>0.9999$
16

\begin{tabular}{ll}
\multicolumn{2}{c}{16} \\
4 weeks & 7 weeks \\
0.3588 & 0.978 \\
0.1044 & $>0.9999$ \\
0.1086 & $>0.9999$ \\
0.3447 & $>0.9999$ \\
0.4631 & $>0.9999$ \\
0.4612 & $>0.9999$ \\
0.3703 & $>0.9999$ \\
0.6739 & 0.6934 \\
0.9974 & 0.8068 \\
0.1335 & $>0.9999$ \\
$>0.9999$ & $>0.9999$ \\
$>0.9999$ & $>0.9999$ \\
&
\end{tabular}

16

4 weeks 7 weeks

$0.2502 \quad 0.9302$

$0.9956 \quad 0.9999$

$>0.9999 \quad 0.8105$

$0.9974 \quad 0.7555$

(Continued)
16

0.7793

0.9855

0.9989

0.9996

0.8846

0.446

0.987

$0.8123 \quad 0.01$

$>0.9999 \quad 0.2806$

$0.5163 \quad 0.7894$

$>0.9999 \quad 0.1785$
7 weeks

0.9878

0.9655

0.0526

0.9163

0.9695

0.8257

0.071

$>0.9999$

0.375

0.9962

$>0.9999$
24

4 weeks 7 weeks

$>0.9999 \quad 0.9931$

$>0.9999 \quad 0.9856$

$>0.9999 \quad 0.9992$

0.9477

$>0.9999$

$>0.9999$

0.9922

0.1943

0.5351

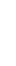

24

7 weeks

0.4134

0.3246

0.149

0.2074

0.92

0.9416

0.6959

0.8289
32

4 weeks 7 weeks

$0.0045 \quad 0.1442$

$0.0179 \quad 0.0068$

$0.0009 \quad 0.0165$

$0.0165 \quad 0.0528$

$0.4277 \quad 0.2029$

$0.2993 \quad 0.3881$

$0.0801 \quad 0.9978$

$0.6623>0.9999$

$0.0182>0.9999$ 
Table 5: Continued

Absolute peak latency $(\mathrm{ms})$

$\begin{array}{llll}12 & 16 & 24 & 32\end{array}$

$\underline{P e a k ~ I ~}$ Frequency $(\mathrm{kHz}) \quad 4$ weeks $\quad 7$ weeks $\quad 4$ weeks $\quad 7$ weeks $\quad 4$ weeks $\quad 7$ weeks $\quad 4$ weeks $\quad 7$ weeks $\quad 4$ weeks 7 weeks 4 weeks 7 weeks $\begin{array}{llllll}25 & 0.9837 & >0.9999 & 0.8812 & >0.9999 & >0.9999\end{array}$

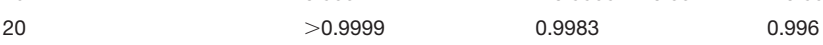

$15>0.9999 \quad 0.2176$

Peak I-III

Frequency (kHz)

Intensity (dB SPL) 80

\section{5}

70

65

60

55

50

45

40

30

25

15

10

Peak I-IV

Frequency $(\mathrm{kHz})$

Intensity (dB SPL) 80

80
75

ak I

Frequency $(\mathrm{kHz})$

Intensity (dB SPL) 80

\section{75}

70

65

60

55

50
45

40

35

30

25

20

15

Peak II

Frequency $(\mathrm{kHz})$
4

4 weeks 7 weeks

$\begin{array}{llll}0.9976 & 0.9757 & 0.9166 & >0.9999\end{array}$

$0.9889>0.9999>0.9999>0.9999$

$0.9949 \quad 0.9968$

$\begin{array}{lll}0.9992 & 0.9974 & 0.982\end{array}$

$>0.9999>0.9999 \quad 0.9933$

$>0.9999>0.9999 \quad 0.9414$

$>0.9999>0.9999 \quad 0.8017$

$\begin{array}{lll}0.3971 & >0.9999 & 0.223\end{array}$

$\begin{array}{lll}0.914 & >0.9999 & 0.0168\end{array}$

0.8953

$>0.9999$

$>0.9999$

0.8909

$>0.9999$

$>0.9999$

4

$\begin{array}{ll}4 \text { weeks } & 7 \text { weeks } \\ 0.1527 & >0.9999\end{array}$

$0.0381>0.9999$

$0.0453 \quad 0.9938$

$0.0512 \quad 0.9986$

$0.0778 \quad 0.3014$

$0.049 \quad 0.9989$

$0.012 \quad 0.9811$

$0.0023 \quad 0.5266$

$0.0465 \quad 0.9852$

0.2528

$>0.9999$

$>0.9999$

0.99

0.8419

$>0.9999$

8

0.0537

0.0292

0.0632

0.4671

$>0.9999$

0.9702

0.9705

0.1471

0.0306

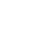

$\begin{array}{lll}0.7248 & >0.9999 & 0.6885\end{array}$

$>0.9999 \quad 0.5053$

$>0.9999 \quad 0.9914$

$>0.9999 \quad 0.9852$

$>0.9999 \quad 0.8667$

$>0.9999 \quad 0.779$

$>0.9999 \quad 0.2776$

$>0.9999 \quad 0.8794$

$>0.9999 \quad 0.6252$

$>0.9999 \quad 0.2341$

$0.9996 \quad 0.8523$

12

16

7

$\begin{array}{lll}>0.9999 & 0.554 & 0.5908\end{array}$

$0.9985 \quad 0.2906$

$>0.9999 \quad 0.1191$

$>0.9999 \quad 0.2001$

0.9987

0.9994

0.9892

0.9981

$>0.9999 \quad 0.9953$

$>0.9999$

$>0.999$

0.9995

$>0.9999$

\section{(1)}

$\begin{array}{ll}4 \text { weeks } & 7 \text { weeks } \\ 0.9999 & >0.9999\end{array}$

$>0.9999>0.9999$

$>0.9999>0.9999$

$0.9999 \quad 0.9999$

$>0.9999 \quad 0.9901$

$>0.9999>0.9999$

$0.9899>0.9999$

$0.407>0.9999$

$0.3773>0.9999$

$0.7805>0.9999$

$0.9858>0.9999$

$>0.9999$

$>0.9999$

$>0.9999$
12

weeks 7 weeks 4 weeks

\subsection{2}

0.0598

0.2666

0.4032

0.3574

0.5349

0.2268

0.9487

0.4967

$>0.9999$

$>0.9999$

0.9985

0.9084

0.9084

4 weeks
0.0656
0.1836
0.0581
0.2361
0.269
0.2612
0.0231
0.0931
0.0268
0.2056
0.0037
0.0482

7

7 weeks

0.9993

0.9992

0.9977

$>0.9999$

$>0.9999$

0.894

0.9748

0.9928

$>0.9999$

$>0.9999$

$>0.9999$

$>0.9999$

0.9977

0.9148

\section{Amplitudes $(\mu \mathrm{V})$}

12

4 weeks 7 weeks 4 weeks

$\begin{array}{lll}0.5909 & 0.9978 & 0.2805\end{array}$

$>0.9999>0.9999$

$>0.9999>0.9999$

$>0.9999>0.9999$

$0.9999 \quad 0.9948$

$>0.9999 \quad 0.9997$

$0.9989 \quad 0.8927$

0.9501

0.9979

$>0.9999 \quad 0.9006$

$>0.9999 \quad 0.8234$

$>0.9999 \quad 0.9977$

$>0.9999$

$>0.9999>0.9999$

$>0.9999>0.9999$

7 weeks

$>0.9999$

$>0.9999$

0.9991

$>0.9999$

$>0.9999$

$>0.9999$

$>0.9999$

$>0.9999$

$>0.9999$

$>0.9999$

$>0.9999$

0.9984

$>0.9999$

0.9635
16

4 weeks 7 weeks

$0.0631 \quad 0.3408$

0.0675

0.0406

0.0834

0.0159

0.0064
0.2159

0.0021

0.1306

0.0093

0.9117

0.9969

0.985

0.1804

0.9933

0.9976

0.5279

0.4367

0.874

0.3928

$>0.9999$
16

4 weeks 7 weeks

$0.9878 \quad 0.9304$

$0.7161>0.9999$

$0.2999>0.9999$

$0.4814>0.9999$

$0.6389>0.9999$

$0.7502>0.9999$

$>0.9999$

$0.9985>0.9999$

$0.999>0.9999$

\begin{tabular}{llll}
\multicolumn{2}{c}{24} & \multicolumn{2}{c}{32} \\
4 weeks & 7 weeks & 4 weeks & 7 weeks \\
0.1734 & $>0.9999$ & $<0.0001$ & 0.992 \\
0.6515 & $>0.9999$ & 0.004 & 0.5808 \\
0.8584 & $>0.9999$ & 0.0076 & 0.9909 \\
0.9199 & $>0.9999$ & 0.0157 & 0.976 \\
0.6087 & $>0.9999$ & 0.2889 & 0.7911 \\
0.9968 & $>0.9999$ & 0.5865 & 0.6075 \\
0.9993 & $>0.9999$ & 0.1887 & 0.9994 \\
0.8109 & 0.8705 & $>0.9999$ & 0.9997 \\
0.5799 & 0.938 & 0.4215 & 0.9471 \\
& 0.9739 & &
\end{tabular}

4 weeks 7 weeks

$0.2903 \quad 0.7543$

$0.5212 \quad 0.8423$

$0.8919 \quad 0.9866$

$>0.9999 \quad 0.9899$

$\begin{array}{ll}0.7358 & 0.2971\end{array}$

$0.9767 \quad 0.887$

$>0.9999 \quad 0.9998$

$>0.9999 \quad 0.9985$

$>0.9999 \quad 0.9871$

0.9265

\begin{tabular}{ll}
\multicolumn{2}{c}{32} \\
4 weeks & 7 weeks \\
0.0342 & 0.8997 \\
0.176 & 0.5412 \\
0.019 & 0.8785 \\
0.0121 & 0.7291 \\
0.7695 & 0.9994 \\
0.9608 & 0.7697 \\
0.8609 & 0.9998 \\
0.9866 & 0.8887 \\
$>0.9999$ & 0.844
\end{tabular}

$>0.9999 \quad 0.844$

\footnotetext{
Intensity (dB SPL) 80
} 4

8 12

24

4 weeks

4

32

0.3953

0.9988

4 weeks 7 weeks

0.9808

$>0.9999$

$0.7775>0.9999$

0.8283

0.9942

$0.9956>0.9999$

0.9728

0.9996

$>0.9999$

$>0.9999$

$0.9998>0.9999-0.9999$ 
Table 5: Continued

Absolute peak latency $(\mathrm{ms})$

\begin{tabular}{|c|c|c|c|c|c|c|c|c|c|c|c|c|c|}
\hline \multirow[b]{2}{*}{ Peak I } & \multirow[b]{2}{*}{ Frequency $(\mathrm{kHz})$} & \multicolumn{2}{|c|}{4} & \multicolumn{2}{|c|}{8} & \multicolumn{2}{|c|}{12} & \multicolumn{2}{|c|}{16} & \multicolumn{2}{|c|}{24} & \multicolumn{2}{|c|}{32} \\
\hline & & 4 weeks & 7 weeks & 4 weeks & 7 weeks & 4 weeks & 7 weeks & 4 weeks & 7 weeks & 4 weeks & 7 weeks & 4 weeks & 7 weeks \\
\hline & 70 & $>0.9999$ & 0.9162 & 0.0546 & 0.8915 & 0.0544 & $>0.9999$ & 0.1351 & 0.9213 & 0.8061 & 0.982 & 0.986 & 0.9787 \\
\hline & 65 & $>0.9999$ & 0.921 & 0.011 & 0.916 & 0.0114 & 0.9853 & 0.263 & 0.7202 & $>0.9999$ & $>0.9999$ & 0.9997 & 0.9996 \\
\hline & 60 & 0.9997 & 0.9992 & 0.0216 & 0.94 & 0.0006 & 0.9953 & 0.3549 & 0.9947 & 0.9995 & $>0.9999$ & 0.9972 & $>0.9999$ \\
\hline & 55 & $>0.9999$ & $>0.9999$ & 0.0685 & 0.9992 & $<0.0001$ & 0.8365 & 0.1353 & $>0.9999$ & $>0.9999$ & $>0.9999$ & $>0.9999$ & $>0.9999$ \\
\hline & 50 & 0.9983 & $>0.9999$ & 0.1122 & 0.9952 & 0.0002 & 0.987 & 0.1224 & $>0.9999$ & $>0.9999$ & $>0.9999$ & $>0.9999$ & $>0.9999$ \\
\hline & 45 & 0.7312 & $>0.9999$ & 0.0227 & $>0.9999$ & 0.0002 & 0.999 & 0.1396 & $>0.9999$ & $>0.9999$ & 0.9996 & $>0.9999$ & 0.9673 \\
\hline & 40 & 0.4724 & 0.9999 & 0.1345 & $>0.9999$ & 0.0001 & $>0.9999$ & 0.3741 & 0.9997 & 0.91 & 0.9753 & $>0.9999$ & 0.998 \\
\hline & 35 & 0.6146 & $>0.9999$ & 0.713 & $>0.9999$ & 0.0131 & $>0.9999$ & 0.4482 & 0.9855 & & 0.9949 & & \\
\hline & 30 & 0.9654 & 0.9977 & & $>0.9999$ & 0.2393 & $>0.9999$ & 0.8649 & $>0.9999$ & & & & \\
\hline & 25 & & $>0.9999$ & & $>0.9999$ & 0.86 & $>0.9999$ & $>0.9999$ & $>0.9999$ & & & & \\
\hline & 20 & & $>0.9999$ & & $>0.9999$ & 0.9986 & $>0.9999$ & & & & & & \\
\hline & 15 & & $>0.9999$ & & & & $>0.9999$ & & & & & & \\
\hline & 10 & & & & & & & & & & & & \\
\hline \multirow[t]{2}{*}{ Peak III } & Frequency $(\mathrm{kHz})$ & \multicolumn{2}{|c|}{4} & \multicolumn{2}{|c|}{8} & \multicolumn{2}{|c|}{12} & \multicolumn{2}{|c|}{16} & \multicolumn{2}{|c|}{24} & \multicolumn{2}{|c|}{32} \\
\hline & & 4 weeks & 7 weeks & 4 weeks & 7 weeks & 4 weeks & 7 weeks & 4 weeks & 7 weeks & 4 weeks & 7 weeks & 4 weeks & 7 weeks \\
\hline \multirow[t]{15}{*}{ Intensity (dB SPL) } & 80 & 0.8773 & 0.9694 & 0.617 & 0.8557 & 0.9879 & 0.9979 & $>0.9999$ & $>0.9999$ & 0.9991 & $>0.9999$ & 0.7727 & 0.9997 \\
\hline & 75 & 0.0467 & 0.9852 & 0.4543 & 0.9824 & 0.9999 & 0.9997 & 0.198 & $>0.9999$ & $>0.9999$ & 0.9937 & $>0.9999$ & $>0.9999$ \\
\hline & 70 & 0.0219 & $>0.9999$ & 0.25 & $>0.9999$ & 0.0625 & $>0.9999$ & 0.0036 & $>0.9999$ & $>0.9999$ & 0.9996 & $>0.9999$ & 0.6554 \\
\hline & 65 & 0.021 & $>0.9999$ & 0.0496 & 0.9989 & 0.0004 & $>0.9999$ & 0.0003 & 0.8926 & $>0.9999$ & $>0.9999$ & 0.971 & 0.9484 \\
\hline & 60 & 0.1528 & 0.809 & 0.0245 & 0.9994 & $<0.0001$ & 0.8908 & 0.0017 & 0.9971 & 0.9999 & $>0.9999$ & $>0.9999$ & 0.9655 \\
\hline & 55 & 0.8908 & 0.9668 & 0.2514 & 0.9982 & 0.0001 & 0.752 & 0.0503 & $>0.9999$ & 0.9949 & 0.9047 & $>0.9999$ & 0.982 \\
\hline & 50 & 0.3084 & $>0.9999$ & 0.718 & 0.9745 & 0.0039 & 0.9942 & 0.753 & 0.9988 & 0.9157 & $>0.9999$ & $>0.9999$ & $>0.9999$ \\
\hline & 45 & 0.5522 & 0.9984 & 0.8589 & 0.8129 & 0.2383 & 0.9858 & $>0.9999$ & 0.9992 & $>0.9999$ & $>0.9999$ & 0.9161 & 0.882 \\
\hline & 40 & 0.5642 & $>0.9999$ & 0.9969 & $>0.9999$ & 0.9678 & $>0.9999$ & $>0.9999$ & 0.9896 & 0.9999 & 0.9408 & 0.9908 & $>0.9999$ \\
\hline & 35 & 0.8501 & 0.9999 & 0.9998 & 0.9994 & 0.9973 & 0.9991 & $>0.9999$ & 0.9882 & & $>0.9999$ & & \\
\hline & 30 & 0.9992 & 0.9996 & & 0.9914 & $>0.9999$ & 0.9423 & $>0.9999$ & $>0.9999$ & & & & \\
\hline & 25 & & 0.9874 & & 0.9937 & $>0.9999$ & $>0.9999$ & $>0.9999$ & $>0.9999$ & & & & \\
\hline & 20 & & $>0.9999$ & & $>0.9999$ & $>0.9999$ & $>0.9999$ & & & & & & \\
\hline & 15 & & $>0.9999$ & & & & $>0.9999$ & & & & & & \\
\hline & 10 & & & & & & & & & & & & \\
\hline \multirow[t]{2}{*}{ Peak IV } & Frequency $(\mathrm{kHz})$ & 4 & \multicolumn{2}{|c|}{8} & & & & & & & & & \\
\hline & & 4 weeks & 7 weeks & 4 weeks & 7 weeks & 4 weeks & 7 weeks & 4 weeks & 7 weeks & 4 weeks & 7 weeks & 4 weeks & 7 weeks \\
\hline Intensity (dB SPL) & 80 & $>0.9999$ & 0.9937 & $>0.9999$ & $>0.9999$ & 0.8751 & $>0.9999$ & $>0.9999$ & 0.9994 & 0.0971 & $>0.9999$ & 0.2365 & 0.8811 \\
\hline & 75 & 0.9989 & 0.9997 & $>0.9999$ & 0.9999 & $>0.9999$ & $>0.9999$ & 0.3295 & 0.9867 & 0.2672 & $>0.9999$ & 0.874 & $>0.9999$ \\
\hline & 70 & 0.2896 & 0.9992 & 0.6309 & 0.9988 & 0.4904 & 0.9969 & 0.0242 & 0.9103 & 0.078 & 0.989 & $>0.9999$ & 0.9995 \\
\hline & 65 & 0.0589 & $>0.9999$ & 0.0544 & $>0.9999$ & 0.2406 & $>0.9999$ & 0.0022 & 0.9991 & 0.2641 & $>0.9999$ & 0.9889 & 0.9989 \\
\hline & 60 & 0.4861 & $>0.9999$ & 0.0043 & $>0.9999$ & 0.0091 & $>0.9999$ & 0.0083 & 0.9603 & 0.5788 & $>0.9999$ & 0.9982 & 0.8113 \\
\hline & 55 & 0.6744 & $>0.9999$ & 0.1152 & $>0.9999$ & 0.0302 & 0.9997 & 0.008 & $>0.9999$ & 0.9929 & $>0.9999$ & 0.7973 & $>0.9999$ \\
\hline & 50 & 0.8413 & $>0.9999$ & 0.7501 & 0.8958 & 0.0483 & 0.9999 & 0.0039 & 0.9123 & 0.9871 & 0.9959 & 0.9664 & 0.9934 \\
\hline & 45 & 0.5569 & $>0.9999$ & 0.1376 & $>0.9999$ & 0.028 & 0.9688 & 0.2175 & $>0.9999$ & 0.8922 & 0.9975 & 0.9352 & $>0.9999$ \\
\hline & 40 & 0.4028 & 0.9885 & 0.7979 & 0.9996 & 0.2109 & $>0.9999$ & 0.2769 & $>0.9999$ & 0.3515 & $>0.9999$ & $>0.9999$ & 0.7964 \\
\hline & 35 & 0.9667 & $>0.9999$ & 0.9995 & $>0.9999$ & 0.4729 & $>0.9999$ & 0.8783 & $>0.9999$ & & $>0.9999$ & & \\
\hline & 30 & & 0.9994 & & $>0.9999$ & 0.9922 & 0.9997 & $>0.9999$ & 0.9866 & & & & \\
\hline & 25 & & 0.9995 & & $>0.9999$ & 0.7008 & $>0.9999$ & & $>0.9999$ & & & & \\
\hline & 20 & & $>0.9999$ & & $>0.9999$ & & $>0.9999$ & & & & & & \\
\hline & 15 & & $>0.9999$ & & & & $>0.9999$ & & & & & & \\
\hline
\end{tabular}

peak III amplitudes at the low and middle frequency levels $(4,8,12$, and $16 \mathrm{kHz}$; Fig. 7C). By seven weeks, peak III amplitudes in treated animals were comparable to controls at all frequencies and intensities, except for $32 \mathrm{kHz}$ where a significant increase in amplitude was detected in the BLZ945treated group (Fig. 7C). Peak IV (Fig. 7D) amplitudes were significantly decreased in BLZ945-trreated animals at four weeks at all frequency levels. By seven weeks, amplitudes at most frequencies recovered, except for $16 \mathrm{kHz}$ where a significant decrease in amplitude was detected in BLZ945treated mice (Fig. 7D). Multiple comparison analyses revealed no difference at any intensity at seven weeks, indicating that peak IV amplitudes were generally recovered. Overall, these data show that at four weeks, peak amplitudes are significantly decreased in BLZ945-treated animals compared with controls (see Tables 4, 5). After prolonged microglial repopulation, there is evidence of recovery of peak amplitudes throughout the auditory brainstem pathways.

\section{Discussion}

In the present study, we used a CSF1R inhibitor to eliminate microglia early in development and then withdrew 
$8 \mathrm{kHz}$

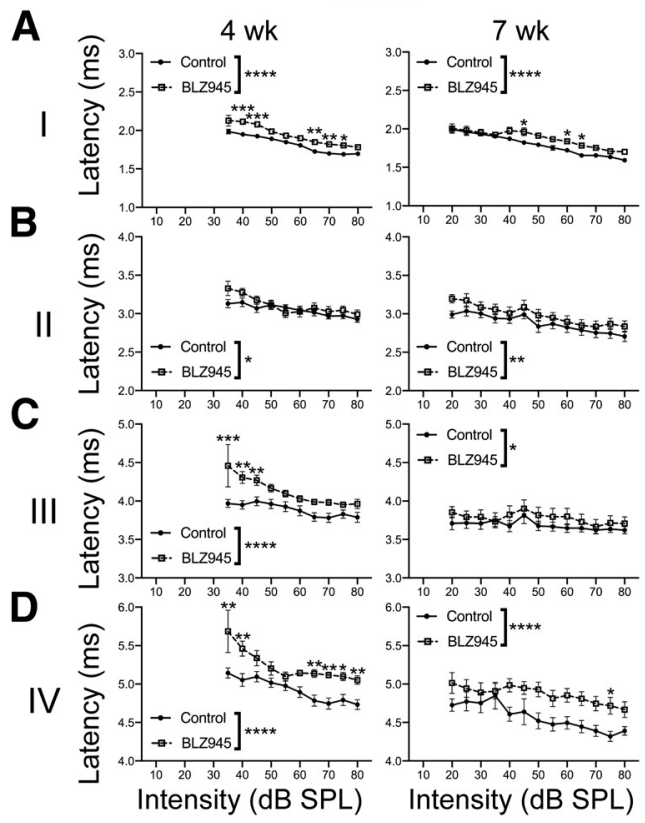

$16 \mathrm{kHz}$
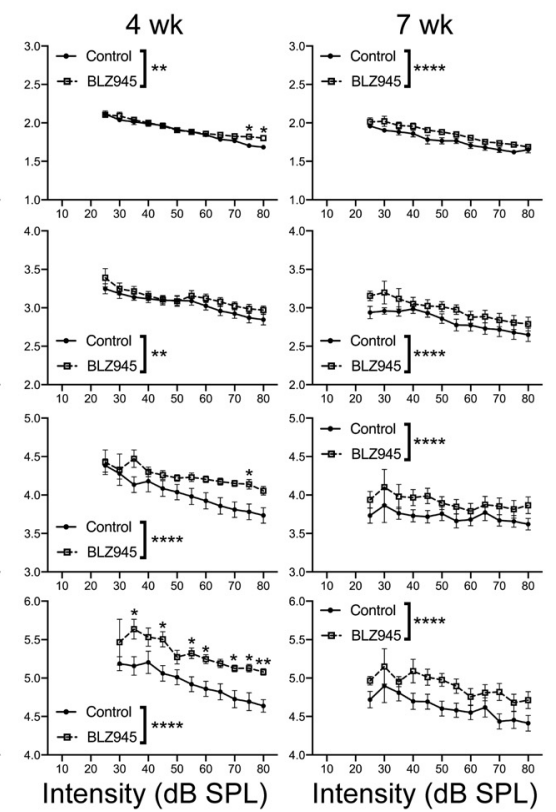
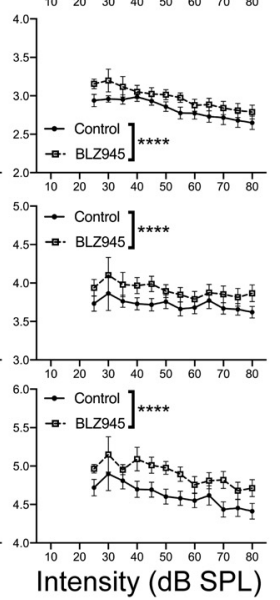

$32 \mathrm{kHz}$

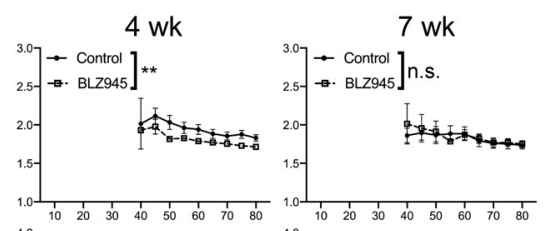

Figure 5. Effect of temporary BLZ945 treatment on ABR peak latencies. A, Absolute peak I latency in response to 8-, 16-, and 32$\mathrm{kHz}$ stimuli at four and seven weeks. Treatment with BLZ945 early in development resulted in significantly increased latency at the low and middle frequencies but significantly decreased latencies at the higher frequencies at four weeks. By seven weeks, the latencies at the lower frequencies remained elevated but latencies at the high frequencies were comparable to control. $\boldsymbol{B}$, Peak II latency was significantly increased in the BLZ945-treated group. Latencies remained elevated by seven weeks at all frequencies except $32 \mathrm{kHz}$. C, BLZ945 treatment resulted in significantly increased peak III latency, with evidence of some recovery by seven weeks in all frequencies. $\boldsymbol{D}$, Significantly increased latencies in peak IV were detected in BLZ945-treated mice in low and middle frequencies at four weeks. Some improvement was detected by seven weeks, but $32-\mathrm{kHz}$ stimuli resulted in significantly longer latency in the BLZ945 group.

this drug to study microglia repopulation and its effects on auditory brainstem circuits. Previously, it was shown that such microglial depletion led to a decrease in GFAP expression as well as retention of polyinnervation in MNTB after the onset of hearing (Milinkeviciute et al., 2019). In this study, we showed that microglia recolonized the brainstem over an extended time course and polyinnervation was present when microglia were still absent from the MNTB at three weeks of age. The majority of MNTB neurons were no longer polyinnervated in BLZ945-treated mice at four weeks of age, a time when microglial numbers were comparable to control levels. Reduced GFAP expression took longer to reach that of controls; however, it was rectified by seven weeks. Functionally, early microglia depletion led to defects in auditory peak latencies and amplitudes, which largely recovered by the later age. This study emphasizes the plasticity of the brain after a challenge to the system and calls attention to the relationship between microglia and auditory function and between different glial cell types.

\section{Microglial repopulation}

Microglia can be eliminated using genetic methods (Bruttger et al., 2015; Wieghofer et al., 2015), pharmacological methods (Pyonteck et al., 2013; Gerber et al., 2018), or irradiation (Simard and Rivest, 2004; Han et al., 2019). We used subcutaneous BLZ945 injections early in development and achieved a complete elimination of IBA1-positive cells in the brainstem. Oral BLZ945 treatment in the five-week cuprizone mouse model resulted in reduced microglial numbers (up to 60\%; Beckmann et al., 2018), highlighting the differences between the delivery method as well as age of the animal and region of the brain analyzed despite of the same inhibitory molecule used. Contrasting results between the two studies also extend to the timing of repopulation. After oral administration of BLZ945, active microglia appeared $3 \mathrm{~d}$ posttreatment and normal numbers were reached at $7 \mathrm{~d}$ (Beckmann et al., 2018). Similarly, microglial depletion with PLX3397 in adult mice resulted in re-emergence of immature microglia $3 \mathrm{~d}$ after the cessation of treatment. These cells gradually acquired adult-like phenotypes and reached control density after $14 \mathrm{~d}$ (Elmore et al., 2014). In our study, microglia did not populate the MNTB until after $18 \mathrm{~d}$ posttreatment, at four weeks of age. The difference in repopulation timing likely lies in our method in elimination of microglia early during postnatal development when microglial cells should be proliferating and populating the entire brain. BLZ945 treatment not only depleted microglia but, in part, prevented them from completing their initial brainstem colonization.

\section{Origins of repopulating microglia}

Microglia have a low turnover rate and can survive throughout the lifespan of an animal (Fuger et al., 2017). They can self-regulate and renew several times throughout life in rodents (Askew et al., 2017; Tay et al., 2017). We successfully eliminated microglia early in 
$8 \mathrm{kHz}$

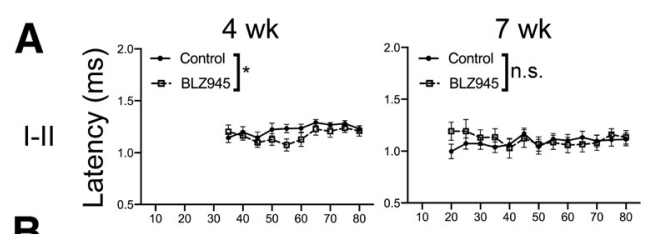

B
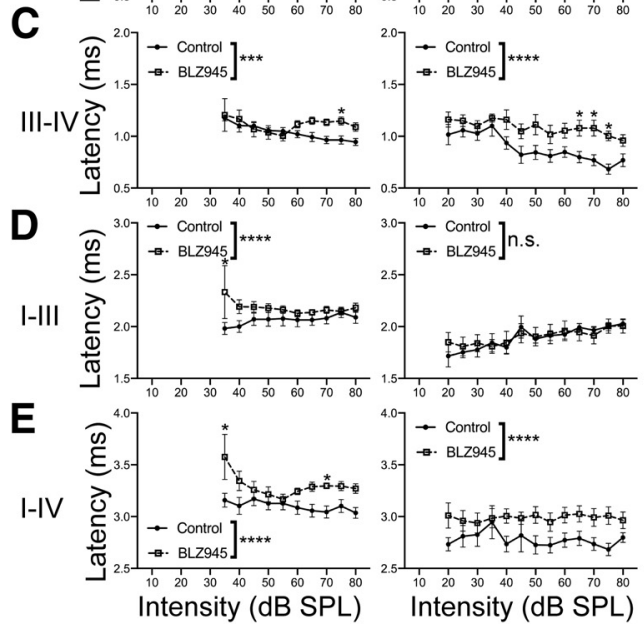

$16 \mathrm{kHz}$

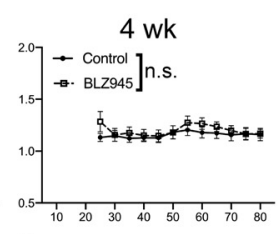

$32 \mathrm{kHz}$
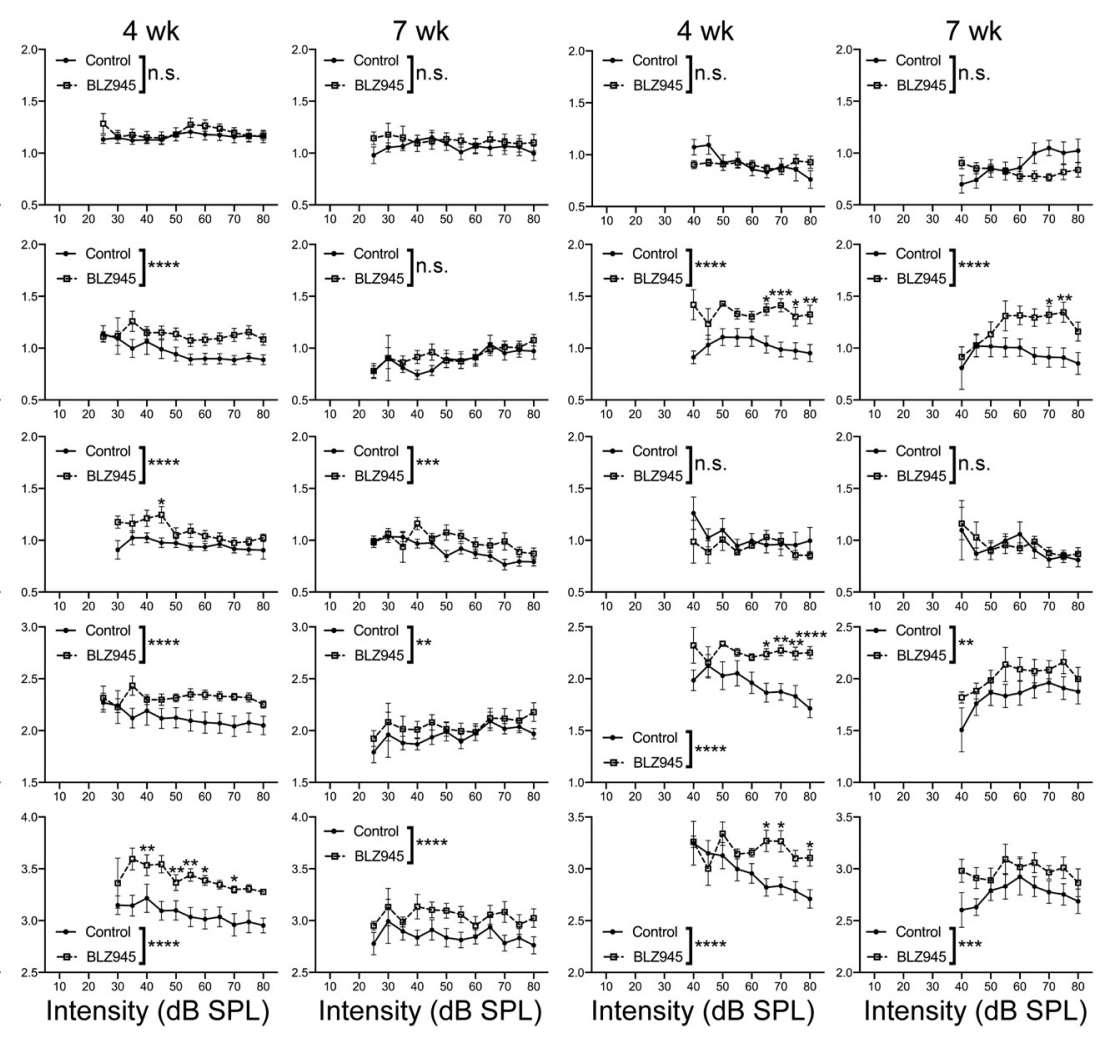

Figure 6. Effect of temporary BLZ945 treatment on ABR interpeak latencies. A, ABR peak I-II interpeak latencies in response to $8 \mathrm{kHz}$ were significantly decreased in BLZ945-treated mice at four weeks. By seven weeks, there were no differences at any frequency level. B, Peak II-III interpeak latency was significantly increased in BLZ945-treated mice at all frequency levels. By seven weeks, low and middle frequencies recovered, but high frequencies remained significantly elevated. C, Peak III-IV interpeak latencies for the BLZ945 group were significantly longer in low and middle frequencies in both ages, while the high frequencies were not affected. $\boldsymbol{D}$, Peak I-III interpeak latency was significantly elevated in BLZ945-treated mice at all frequency levels at four weeks. By seven weeks, only the low frequency was comparable to the control group. $\boldsymbol{E}$, BLZ945 treatment resulted in significantly elevated peak I-IV interpeak latency at all frequencies in both ages.

development and observed that, after cessation of treatment, microglia repopulate the brainstem. The origins of the reappearing brainstem microglia are not known. Studies implicate that repopulation can be achieved in one of three ways - proliferation of remaining microglia, stimulation of microglial precursors or infiltration of peripheral monocytes (for review, see Han et al., 2019). The process of repopulation likely depends on the method of depletion. It is possible that irradiation leads to an influx of macrophages from the periphery because of the disruption of the blood-brain barrier (BBB; Simard and Rivest, 2004). On the other hand, pharmacological depletion with drug-infused rodent diet could induce the proliferation from resident microglial progenitor cells such as nestin positive cells (Elmore et al., 2014). Additionally, proliferation of surviving microglial cells could support the microglial replenishment in the CNS. Genetic ablation of microglia (80\%) using the Cx3cr1 ${ }^{\text {CreER }}$ based system showed that repopulating microglial cells arose exclusively from an internal CNS-resident cell pool (Bruttger et al., 2015; Askew et al., 2017; Jakel and Dimou, 2017; Huang et al., 2018). Earlier it was shown that repeated subcutaneous BLZ945 injections resulted in complete microglial elimination in the brainstem (Milinkeviciute et al., 2019), thus, new cells do not likely emerge from surviving microglia. Peripheral macrophages could be another origin if the BBB was disrupted; however, despite the evidence that BLZ945 crosses the BBB (Pyonteck et al., 2013; Beckmann et al., 2018), there are no reports about disruption of the BBB. Consequently, CNS resident progenitors might be recruited to replenish the microglial cell pool after BLZ945 treatment is stopped. These studies support the view that the course of repopulation highly depends on the depletion approach used and the level of microglial elimination.

\section{Microglia and calyceal pruning}

Microglia are continuously surveying their environment by extending and retracting their processes (Nimmerjahn et al., 2005). During development, microglia have been shown to have a significant role in the formation of neural circuits (Miyamoto et al., 2016; Reemst et al., 2016; Nelson and Lenz, 2017; Basilico et al., 2019; Nonaka and Nakanishi, 2019) in health and pathologic conditions (Neniskyte and Gross, 2017; Wolf et al., 2017; Whitelaw, 


\section{$8 \mathrm{kHz}$}

$16 \mathrm{kHz}$

$32 \mathrm{kHz}$

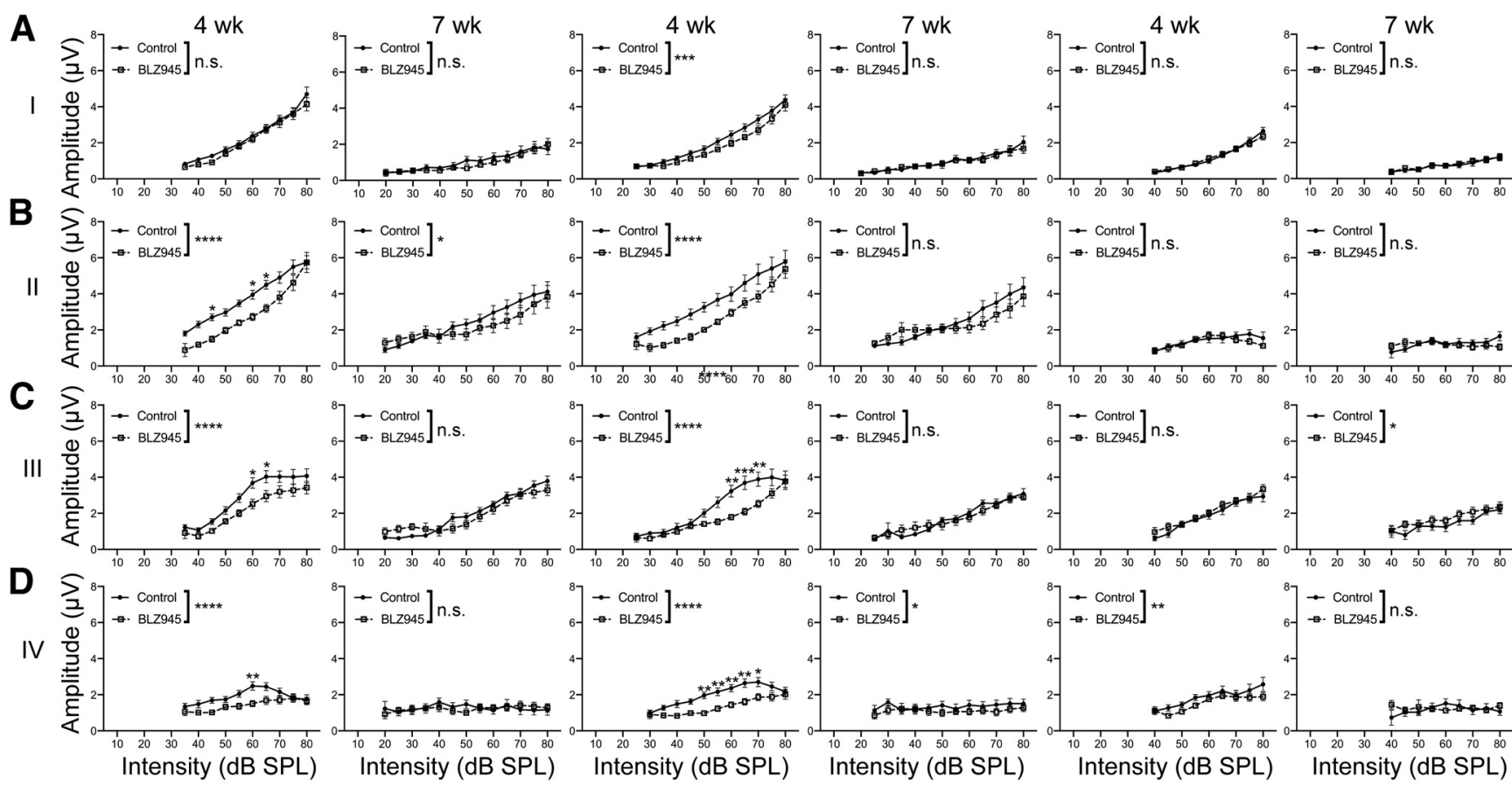

Figure 7. Effect of temporary BLZ945 treatment on ABR amplitudes. $\boldsymbol{A}$, Trough to peak amplitudes $(\mu \mathrm{V})$ of $\mathrm{ABR}$ peak I in response to $8-, 16-$, and $32-\mathrm{kHz}$ stimuli at four and seven weeks. Amplitudes were comparable to control at most frequency levels except $16 \mathrm{kHz}$, where amplitudes were significantly diminished. B. Peak II amplitude was significantly decreased in the BLZ945-treated group at the low and middle frequencies, with some improvement by seven weeks. $\boldsymbol{C}$, Peak III amplitudes were significantly lower in the BLZ945 mice at low and middle frequencies at four weeks, and recovered by seven weeks. The higher frequency showed that peak III amplitude was significantly elevated in the BLZ945-treated group at seven weeks. D, BLZ945-treated mice showed significantly decreased peak IV amplitudes at all frequency levels at four weeks. By seven weeks, peak IV amplitude was comparable to control at 8 and $32 \mathrm{kHz}$.

2018). Microglia can eliminate synapses (Paolicelli et al., 2011; Schafer et al., 2012) or promote their formation (Schafer et al., 2012; Parkhurst et al., 2013; Miyamoto et al., 2016). In MNTB, microglia appear at the time coinciding with intense synaptic development. They peak in number at around P14 and a slight decline is observed at P23 (Dinh et al., 2014). We noticed a further significant reduction in microglial areal coverage between weeks 4 and 7 . This change may be reflective of a shift in the function of microglia from a developmental role to a homeostatic role.

In the auditory brainstem, microglial elimination resulted in increased numbers of polyinnervated neurons remaining in the MNTB after hearing onset (Milinkeviciute et al., 2019). It was not clear, however, whether the calyceal pruning was delayed or directly related to the absence of microglia from MNTB. Here, we found that even when treatment is stopped at P10 and microglia are allowed to populate the brain, innervation of principal MNTB neurons remains abnormal until microglia inhabit the nucleus. It has been shown that microglia promote brain repair after injury (Willis et al., 2020). In our case there was no injury, and microglia appeared in the MNTB between weeks 3 and 4 for the first time. It appears that newly emerged microglia initialized the developmental process that normally occurs before the onset of hearing. Depletion and subsequent recolonization of microglia in the retina showed restoration of functions of endogenous microglia in homeostasis (Zhang et al., 2018). It is important to note that calyces of Held developed normally in terms of their surface area and complexity; however, the pruning of additional calyces was impaired, indicating that there may be different mechanisms that regulate these two processes. These findings suggest a correlation between microglial presence and pruning of calyces of Held in MNTB.

\section{Microglia-astrocyte communication}

Crosstalk between glial cells is essential for brain development and function in physiological and pathologic conditions. Bidirectional communication between microglia and astrocytes is established through cytokines, chemokines, ATP and growth factors early in development when they first populate the brain parenchyma (Jha et al., 2016; Liddelow et al., 2017; Vainchtein et al., 2018). Previously, it was shown that GFAP expression is reduced after microglia depletion (Milinkeviciute et al., 2019). Here, we found restoration of GFAP expression to control levels only after prolonged microglial repopulation. In contrast, oral BLZ945 treatment in the five-week cuprizone mouse model increased the number of GFAP-positive 
and ALDH1L1-positive astrocytes in the cortex, striatum, and corpus callosum (Beckmann et al., 2018). Similarly, PLX3397 treatment in adult mice resulted in a significant increase in GFAP mRNA (Elmore et al., 2014; Jin et al., 2017). This discrepancy may be attributed to the depletion method used; the delivery method; the developmental stage of the animal at the time of treatment; and/or the area of the brain analyzed. Here, microglia were depleted at the time when the brainstem should be undergoing the initial colonization by microglia (Dinh et al., 2014). This process was halted by injection of CSF1R inhibitor before microglia had a chance to occupy their positions and mature within the auditory brainstem. Given that GFAP is suggested to be a marker of mature (Wofchuk and Rodnight, 1995; Gomes et al., 1999; Middeldorp and Hol, 2011) astrocytes, it is possible that astrocytic maturation is impeded by microglial absence and when microglia are permitted to repopulate, GFAP expression is initiated and becomes comparable to that of control at seven weeks. It is yet to be determined which molecules may be involved in the process of astrocytic maturation.

\section{Auditory brainstem function}

Auditory function requires precision in the development of neuronal circuits as well as accurate organization of specialized synapses. Microglia are known to be necessary for the formation of some neural circuits (Miyamoto et al., 2016; Basilico et al., 2019; Nonaka and Nakanishi, 2019). We thus examined the overall effect of microglia depletion on the auditory function. We found that temporary depletion of microglia in the brainstem early in development resulted in deficits in the ABR. BLZ945 treatment during the first postnatal week caused a moderate hearing loss but thresholds recovered by seven weeks, while click thresholds did not show recovery at this later time period. We also found that BLZ945 treatment had a significant effect on absolute peak latencies as well as interpeak latencies at most frequencies tested, and at seven weeks, these latency defects were still mostly present. However, the diminished amplitudes observed at four weeks in BLZ945-treated mice showed almost full recovery by seven weeks.

Our observation of elevated ABR thresholds could indicate a peripheral defect in treated mice. Defective CSF1R signaling affects bone resorption and leads to abnormalities in the middle and inner ears (Aharinejad et al., 1999; Kanzaki et al., 2011; Okano and Kishimoto, 2019). In the cochlea, CSF1R-positive macrophages are found in the spiral ligament, spiral ganglion and stria vascularis (Okano et al., 2008; Okano and Kishimoto, 2019) while centrally, microglia rely on CSF1 signaling for survival (Ginhoux et al., 2010; Erblich et al., 2011). Thus, treatment with CSF1R inhibitor can affect many areas in the auditory pathway. Our ABR results are consistent with hearing deficits reported in $\mathrm{Csf1}{ }^{\mathrm{op} / \mathrm{op}}$ mice where auditory thresholds were significantly elevated likely because of bone deformities and subsequent conductive hearing loss (Aharinejad et al., 1999; Okano and Kishimoto, 2019). We observed delayed absolute latencies in subsequent peaks as well as interpeak latencies, consistent with brainstem auditory evoked potentials recorded in Csf1 ${ }^{\text {op/op }}$ mice (Michaelson et al., 1996). Central defects could result in part from impaired peripheral function.

In addition to potential peripheral effects, our ABR analyses suggest that BLZ945 may produce central effects independently through its elimination of microglia. We observed significant increases in interpeak latencies in treated animals. A minimal effect was seen for peak I-II, but greater effects were seen for all of the central intervals. These delays were partially corrected after microglia repopulation. Peak III corresponds to the superior olivary complex and reflects in part activity in MNTB. However, it is not known to what extent calyceal pruning directly influences the latency or amplitudes of this peak in our study. Nevertheless, the observation of increased interpeak latency, impaired pruning, and delayed astrocyte maturation in MNTB together support a central effect of microglia elimination on auditory function.

Microglia have been shown to communicate with other glial cells, including oligodendrocytes (Lloyd et al., 2017) and NG2 cells (Müller et al., 2009; Giera et al., 2018) and regulate oligodendrocyte differentiation (Miron and Franklin, 2014). Microglial depletion has revealed a microglial role in the development and maintenance of oligodendrocytes and their progenitors (Hagemeyer et al., 2017). Oligodendrocytes are responsible for myelination of axons and, thus regulate the precise signal conduction seen in auditory pathways (Seidl, 2014; Sinclair et al., 2017; Long et al., 2018). In the MNTB, myelination starts by P9 (Saliu et al., 2014) and increases around the time of ear canal opening (Sinclair et al., 2017). Taken together, microglial elimination may affect conduction velocity (Sinclair et al., 2017).

We showed that treatment with CSF1R inhibitor temporarily reduced peak amplitudes, especially the central peaks. Thus, there are both peripheral and central defects in the ABR. On one hand, bone deformities in the middle and inner ear (Aharinejad et al., 1999; Kanzaki et al., 2011; Okano and Kishimoto, 2019) could have contributed to these impairments which then propagated to the central auditory system, however, we did not test this hypothesis directly. On the other hand, centrally, oligodendrocytes are known to detect and respond to neuronal activity (Gibson et al., 2014) as well as secrete neurotrophic factors. In the auditory brainstem, lack of BDNF secreted by oligodendrocytes significantly reduced amplitudes of ABR peaks II-IV in response to click stimuli (Jang et al., 2019). NG2 cells near calyces of Held were suggested to participate in regulating the fast-signaling properties of calyceal inputs (Müller et al., 2009). Thus, microglial elimination could have affected oligodendrocytes or NG2 cells, leading to impaired neuronal activity and synaptic synchrony which resulted in the reduction of amplitudes we observed.

The observed decrease in ABR amplitudes may be also related to delayed maturation of GFAP positive astrocytes. Astrocytes are highly fenestrated and interspersed between the branches of calyces (Ford et al., 2009; 
Holcomb et al., 2013). GFAP astrocytes express glutamate transporters and regulate glutamate uptake activity (Bergles and Jahr, 1997; Matthias et al., 2003), and are crucial for its recycling (Pow and Robinson, 1994; Laake et al., 1995; Hertz et al., 1999). These glial cells respond to glutamate release from the calyx of Held, which leads to the uptake of excess glutamate and prevention of glutamate receptor saturation, especially in the immature calyx (Bergles and Jahr, 1997; Matthias et al., 2003). In this paper, we showed that temporary microglia depletion during postnatal development reduced GFAP + astrocyte levels. It is thus possible that delayed astrocytic maturation led to impairment in glutamate uptake and allowed for glutamate receptor saturation, and subsequent decrease in ABR amplitudes of the central peaks.

At this point, we cannot predict what exactly causes deterioration of different aspects and parts of ABRs as microglia are found through the auditory system. It is possible that macrophages in the cochlea and microglia in different auditory nuclei have slightly different functions. For peak I, which reflects peripheral activity, frequency-specific effects could reflect tonotopic differences in the cochlea of molecules that interact with macrophages. Centrally, there may also be differences in the way tonotopic regions of individual nuclei interact with glial cells.

Because of the fact that ABR peak III encompasses the auditory response of the entire SOC, we cannot correlate anatomic abnormalities observed in MNTB with ABR results. Rather, we can conclude that temporary loss of CSF1R signaling affects conduction velocity and strength throughout the ascending auditory pathway.

In conclusion, microglia function in a myriad of processes ranging from immune responses to synaptogenesis in the CNS. We eliminated this major cell population and showed remarkable plasticity in the developing auditory brainstem. Not only do microglia completely recolonize the brainstem, but repopulation rectifies previously observed anatomic defects. We showed that microglial presence in MNTB is needed in order for calyceal pruning to take place and for full establishment of the GFAP-positive astrocytic population. Importantly, microglial absence postnatally has a significant impact on auditory function, which partially recovers after microglia return.

\section{References}

Aharinejad S, Grossschmidt K, Franz P, Streicher J, Nourani F, Mackay CA, Firbas W, Plenk H Jr, Marks SC Jr (1999) Auditory ossicle abnormalities and hearing loss in the toothless (osteopetrotic) mutation in the rat and their improvement after treatment with colony-stimulating factor-1. J Bone Miner Res 14:415-423.

Askew K, Li K, Olmos-Alonso A, Garcia-Moreno F, Liang Y, Richardson P, Tipton T, Chapman MA, Riecken K, Beccari S, Sierra A, Molnar Z, Cragg MS, Garaschuk O, Perry VH, GomezNicola D (2017) Coupled proliferation and apoptosis maintain the rapid turnover of microglia in the adult brain. Cell Rep 18:391-405.

Basilico B, Pagani F, Grimaldi A, Cortese B, Di Angelantonio S, Weinhard L, Gross C, Limatola C, Maggi L, Ragozzino D (2019) Microglia shape presynaptic properties at developing glutamatergic synapses. Glia 67:53-67.
Beckmann N, Giorgetti E, Neuhaus A, Zurbruegg S, Accart N, Smith P, Perdoux J, Perrot L, Nash M, Desrayaud S, Wipfli P, Frieauff W, Shimshek DR (2018) Brain region-specific enhancement of remyelination and prevention of demyelination by the CSF1R kinase inhibitor BLZ945. Acta Neuropathol Commun 6:9.

Bergles DE, Jahr CE (1997) Synaptic activation of glutamate transporters in hippocampal astrocytes. Neuron 19:1297-1308.

Bialas AR, Stevens B (2013) TGF-beta signaling regulates neuronal C1q expression and developmental synaptic refinement. Nat Neurosci 16:1773-1782.

Billups B (2005) Colocalization of vesicular glutamate transporters in the rat superior olivary complex. Neurosci Lett 382:66-70.

Bogaerts S, Clements JD, Sullivan JM, Oleskevich S (2009) Automated threshold detection for auditory brainstem responses: comparison with visual estimation in a stem cell transplantation study. BMC Neurosci 10:104.

Borst JG, Sakmann B (1996) Calcium influx and transmitter release in a fast CNS synapse. Nature 383:431-434.

Boudreau JC, Tsuchitani C (1968) Binaural interaction in the cat superior olive S segment. J Neurophysiol 31:442-454.

Brawer JR, Morest DK (1975) Relations between auditory nerve endings and cell types in the cat's anteroventral cochlear nucleus seen with the Golgi method and Nomarski optics. J Comp Neurol 160:491-506.

Bruttger J, Karram K, Wortge S, Regen T, Marini F, Hoppmann N, Klein M, Blank T, Yona S, Wolf Y, Mack M, Pinteaux E, Muller W, Zipp F, Binder H, Bopp T, Prinz M, Jung S, Waisman A (2015) Genetic cell ablation reveals clusters of local self-renewing microglia in the mammalian central nervous system. Immunity 43:92106.

DeHoff RT (1978) Stereological uses of the area tangent count. In: Geometrical probability and biological structures: Buffon's 200th anniversary (Miles RE and Serra J, eds). Berlin: Springer.

Dinh ML, Koppel SJ, Korn MJ, Cramer KS (2014) Distribution of glial cells in the auditory brainstem: normal development and effects of unilateral lesion. Neuroscience 278:237-252.

Elmore MR, Najafi AR, Koike MA, Dagher NN, Spangenberg EE, Rice RA, Kitazawa M, Matusow B, Nguyen H, West BL, Green KN (2014) Colony-stimulating factor 1 receptor signaling is necessary for microglia viability, unmasking a microglia progenitor cell in the adult brain. Neuron 82:380-397.

Eng LF, Ghirnikar RS (1994) GFAP and astrogliosis. Brain Pathol 4:229-237.

Erblich B, Zhu L, Etgen AM, Dobrenis K, Pollard JW (2011) Absence of colony stimulation factor-1 receptor results in loss of microglia, disrupted brain development and olfactory deficits. PLoS One 6: e26317.

Ford MC, Grothe B, Klug A (2009) Fenestration of the calyx of Held occurs sequentially along the tonotopic axis, is influenced by afferent activity, and facilitates glutamate clearance. J Comp Neurol 514:92-106.

Fuentes-Santamaria V, Alvarado JC, Melgar-Rojas P, Gabaldon-UII MC, Miller JM, Juiz JM (2017) The role of glia in the peripheral and central auditory system following noise overexposure: contribution of TNF-alpha and IL-1beta to the pathogenesis of hearing loss. Front Neuroanat 11:9.

Fuger P, Hefendehl JK, Veeraraghavalu K, Wendeln AC, Schlosser C, Obermuller U, Wegenast-Braun BM, Neher JJ, Martus P, Kohsaka S, Thunemann M, Feil R, Sisodia SS, Skodras A, Jucker M (2017) Microglia turnover with aging and in an Alzheimer's model via long-term in vivo single-cell imaging. Nat Neurosci 20:1371-1376.

Gerber YN, Saint-Martin GP, Bringuier CM, Bartolami S, Goze-Bac C, Noristani HN, Perrin FE (2018) CSF1R inhibition reduces microglia proliferation, promotes tissue preservation and improves motor recovery after spinal cord injury. Front Cell Neurosci 12:368.

Gibson EM, Purger D, Mount CW, Goldstein AK, Lin GL, Wood LS, Inema I, Miller SE, Bieri G, Zuchero JB, Barres BA, Woo PJ, Vogel $\mathrm{H}$, Monje $\mathrm{M}$ (2014) Neuronal activity promotes oligodendrogenesis 
and adaptive myelination in the mammalian brain. Science 344:1252304.

Giera S, Luo R, Ying Y, Ackerman SD, Jeong SJ, Stoveken HM, Folts CJ, Welsh CA, Tall GG, Stevens B, Monk KR, Piao X (2018) Microglial transglutaminase-2 drives myelination and myelin repair via GPR56/ADGRG1 in oligodendrocyte precursor cells. Elife 7: e33385.

Ginhoux F, Greter M, Leboeuf M, Nandi S, See P, Gokhan S, Mehler MF, Conway SJ, Ng LG, Stanley ER, Samokhvalov IM, Merad M (2010) Fate mapping analysis reveals that adult microglia derive from primitive macrophages. Science 330:841-845.

Gomes FC, Paulin D, Moura Neto V (1999) Glial fibrillary acidic protein (GFAP): modulation by growth factors and its implication in astrocyte differentiation. Braz J Med Biol Res 32:619-631.

Grande G, Negandhi J, Harrison RV, Wang LY (2014) Remodelling at the calyx of Held-MNTB synapse in mice developing with unilateral conductive hearing loss. J Physiol 592:1581-1600.

Grothe B (2003) New roles for synaptic inhibition in sound localization. Nat Rev Neurosci 4:540-550.

Grothe B, Pecka M, Mcalpine D (2010) Mechanisms of sound localization in mammals. Physiol Rev 90:983-1012.

Hagemeyer N, Hanft KM, Akriditou MA, Unger N, Park ES, Stanley ER, Staszewski O, Dimou L, Prinz M (2017) Microglia contribute to normal myelinogenesis and to oligodendrocyte progenitor maintenance during adulthood. Acta Neuropathol 134:441-458.

Han J, Zhu K, Zhang XM, Harris RA (2019) Enforced microglial depletion and repopulation as a promising strategy for the treatment of neurological disorders. Glia 67:217-231.

Held H (1893) Die centrale Gehorleitung. Arch Anat Physiol 201-248.

Henry KR (1979) Auditory brainstem volume-conducted responses: origins in the laboratory mouse. J Am Aud Soc 4:173-178.

Hertz L, Dringen R, Schousboe A, Robinson SR (1999) Astrocytes: glutamate producers for neurons. J Neurosci Res 57:417-428.

Hoffpauir BK, Grimes JL, Mathers PH, Spirou GA (2006) Synaptogenesis of the calyx of Held: rapid onset of function and one-to-one morphological innervation. J Neurosci 26:5511-5523.

Holcomb PS, Hoffpauir BK, Hoyson MC, Jackson DR, Deerinck TJ, Marrs GS, DeHoff M, Wu J, Ellisman MH, Spirou GA (2013) Synaptic inputs compete during rapid formation of the calyx of Held: a new model system for neural development. J Neurosci 33:12954-12969.

Huang Y, Xu Z, Xiong S, Sun F, Qin G, Hu G, Wang J, Zhao L, Liang Yx, Wu T, Lu Z, Humayun MS, So KF, Pan Y, Li N, Yuan TF, Rao Y, Peng B (2018) Repopulated microglia are solely derived from the proliferation of residual microglia after acute depletion. Nat Neurosci 21:530-540.

Jakel S, Dimou L (2017) Glial cells and their function in the adult brain: a journey through the history of their ablation. Front Cell Neurosci 11:24.

Jang M, Gould E, Xu J, Kim EJ, Kim JH (2019) Oligodendrocytes regulate presynaptic properties and neurotransmission through BDNF signaling in the mouse brainstem. Elife 8:e42156.

Jewett DL, Williston JS (1971) Auditory-evoked far fields averaged from the scalp of humans. Brain 94:681-696.

Jewett DL, Romano MN, Williston JS (1970) Human auditory evoked potentials: possible brain stem components detected on the scalp. Science 167:1517-1518.

Jha MK, Lee WH, Suk K (2016) Functional polarization of neuroglia: implications in neuroinflammation and neurological disorders. Biochem Pharmacol 103:1-16.

Jha MK, Jo M, Kim JH, Suk K (2019) Microglia-astrocyte crosstalk: an intimate molecular conversation. Neuroscientist 25:227-240.

Jin WN, Shi SX, Li Z, Li M, Wood K, Gonzales RJ, Liu Q (2017) Depletion of microglia exacerbates postischemic inflammation and brain injury. J Cereb Blood Flow Metab 37:2224-2236.

Kandler K, Friauf E (1993) Pre- and postnatal development of efferent connections of the cochlear nucleus in the rat. J Comp Neurol 328:161-184.
Kanzaki S, Takada Y, Niida S, Takeda Y, Udagawa N, Ogawa K, Nango N, Momose A, Matsuo K (2011) Impaired vibration of auditory ossicles in osteopetrotic mice. Am J Pathol 178:1270-1278.

Kettenmann H, Kirchhoff F, Verkhratsky A (2013) Microglia: new roles for the synaptic stripper. Neuron 77:10-18.

Kuwabara N, Zook JM (1991) Classification of the principal cells of the medial nucleus of the trapezoid body. J Comp Neurol 314:707-720.

Laake JH, Slyngstad TA, Haug FM, Ottersen OP (1995) Glutamine from glial cells is essential for the maintenance of the nerve terminal pool of glutamate: immunogold evidence from hippocampal slice cultures. J Neurochem 65:871-881.

Lauer AM, Connelly CJ, Graham H, Ryugo DK (2013) Morphological characterization of bushy cells and their inputs in the laboratory mouse (Mus musculus) anteroventral cochlear nucleus. PLoS One 8:e73308.

Liddelow SA, Guttenplan KA, Clarke LE, Bennett FC, Bohlen CJ, Schirmer L, Bennett ML, Munch AE, Chung WS, Peterson TC, Wilton DK, Frouin A, Napier BA, Panicker N, Kumar M, Buckwalter MS, Rowitch DH, Dawson VL, Dawson TM, Stevens B, et al. (2017) Neurotoxic reactive astrocytes are induced by activated microglia. Nature 541:481-487.

Lloyd AF, Davies CL, Miron VE (2017) Microglia: origins, homeostasis, and roles in myelin repair. Curr Opin Neurobiol 47:113-120.

Long P, Wan G, Roberts MT, Corfas G (2018) Myelin development, plasticity, and pathology in the auditory system. Dev Neurobiol 78:80-92.

Matthias K, Kirchhoff F, Seifert G, Huttmann K, Matyash M, Kettenmann H, Steinhauser C (2003) Segregated expression of AMPA-type glutamate receptors and glutamate transporters defines distinct astrocyte populations in the mouse hippocampus. $J$ Neurosci 23:1750-1758.

Michaelson MD, Bieri PL, Mehler MF, Xu H, Arezzo JC, Pollard JW, Kessler JA (1996) CSF-1 deficiency in mice results in abnormal brain development. Development 122:2661-2672.

Middeldorp J, Hol EM (2011) GFAP in health and disease. Prog Neurobiol 93:421-443.

Milinkeviciute G, Henningfield CM, Muniak MA, Chokr SM, Green KN, Cramer KS (2019) Microglia regulate pruning of specialized synapses in the auditory brainstem. Front Neural Circuits 13:55.

Miron VE, Franklin RJ (2014) Macrophages and CNS remyelination. J Neurochem 130:165-171.

Miyamoto A, Wake H, Ishikawa AW, Eto K, Shibata K, Murakoshi H, Koizumi S, Moorhouse AJ, Yoshimura Y, Nabekura J (2016) Microglia contact induces synapse formation in developing somatosensory cortex. Nat Commun 7:12540.

Morest DK (1968) The growth of synaptic endings in the mammalian brain: a study of the calyces of the trapezoid body. Z Anat Entwicklungsgesch 127:201-220.

Müller J, Reyes-Haro D, Pivneva T, Nolte C, Schaette R, Lübke J, Kettenmann H (2009) The principal neurons of the medial nucleus of the trapezoid body and NG2(+) glial cells receive coordinated excitatory synaptic input. J Gen Physiol 134:115-127.

Muniak MA, Ayeni FE, Ryugo DK (2018) Hidden hearing loss and endbulbs of Held: evidence for central pathology before detection of ABR threshold increases. Hear Res 364:104-117.

Nelson LH, Lenz KM (2017) Microglia depletion in early life programs persistent changes in social, mood-related, and locomotor behavior in male and female rats. Behav Brain Res 316:279-293.

Neniskyte U, Gross CT (2017) Errant gardeners: glial-cell-dependent synaptic pruning and neurodevelopmental disorders. Nat Rev Neurosci 18:658-670.

Nimmerjahn A, Kirchhoff F, Helmchen F (2005) Resting microglial cells are highly dynamic surveillants of brain parenchyma in vivo. Science 308:1314-1318.

Nonaka S, Nakanishi H (2019) Microglial clearance of focal apoptotic synapses. Neurosci Lett 707:134317.

Okano T, Kishimoto I (2019) Csf1 signaling regulates maintenance of resident macrophages and bone formation in the mouse cochlea. Front Neurol 10:1244. 
Okano T, Nakagawa T, Kita T, Kada S, Yoshimoto M, Nakahata T, Ito $J(2008)$ Bone marrow-derived cells expressing lba1 are constitutively present as resident tissue macrophages in the mouse cochlea. J Neurosci Res 86:1758-1767.

Paolicelli RC, Bolasco G, Pagani F, Maggi L, Scianni M, Panzanelli P, Giustetto M, Ferreira TA, Guiducci E, Dumas L, Ragozzino D, Gross CT (2011) Synaptic pruning by microglia is necessary for normal brain development. Science 333:1456-1458.

Parkhurst CN, Yang G, Ninan I, Savas JN, Yates JR 3rd, Lafaille JJ, Hempstead BL, Littman DR, Gan WB (2013) Microglia promote learning-dependent synapse formation through brain-derived neurotrophic factor. Cell 155:1596-1609.

Picton TW, Hillyard SA, Krausz HI, Galambos R (1974) Human auditory evoked potentials. I. Evaluation of components. Electroencephalogr Clin Neurophysiol 36:179-190.

Pow DV, Robinson SR (1994) Glutamate in some retinal neurons is derived solely from glia. Neuroscience 60:355-366.

Pyonteck SM, Akkari L, Schuhmacher AJ, Bowman RL, Sevenich L, Quail DF, Olson OC, Quick ML, Huse JT, Teijeiro V, Setty M, Leslie CS, Oei Y, Pedraza A, Zhang J, Brennan CW, Sutton JC, Holland EC, Daniel D, Joyce JA (2013) CSF-1R inhibition alters macrophage polarization and blocks glioma progression. Nat Med 19:1264-1272.

Reemst K, Noctor SC, Lucassen PJ, Hol EM (2016) The indispensable roles of microglia and astrocytes during brain development. Front Hum Neurosci 10:566.

Ryugo DK, Sento S (1991) Synaptic connections of the auditory nerve in cats: relationship between endbulbs of held and spherical bushy cells. J Comp Neurol 305:35-48.

Saliu A, Adise S, Xian S, Kudelska K, Rodríguez-Contreras A (2014) Natural and lesion-induced decrease in cell proliferation in the medial nucleus of the trapezoid body during hearing development. $J$ Comp Neurol 522:971-985.

Sanes DH, Rubel EW (1988) The ontogeny of inhibition and excitation in the gerbil lateral superior olive. J Neurosci 8:682-700.

Sätzler K, Söhl LF, Bollmann JH, Borst JG, Frotscher M, Sakmann B, Lübke JH (2002) Three-dimensional reconstruction of a calyx of Held and its postsynaptic principal neuron in the medial nucleus of the trapezoid body. J Neurosci 22:10567-10579.

Schafer DP, Stevens B (2010) Synapse elimination during development and disease: immune molecules take centre stage. Biochem Soc Trans 38:476-481.

Schafer DP, Lehrman EK, Kautzman AG, Koyama R, Mardinly AR, Yamasaki R, Ransohoff RM, Greenberg ME, Barres BA, Stevens B (2012) Microglia sculpt postnatal neural circuits in an activity and complement-dependent manner. Neuron 74:691-705.

Schindelin J, Arganda-Carreras I, Frise E, Kaynig V, Longair M, Pietzsch T, Preibisch S, Rueden C, Saalfeld S, Schmid B, Tinevez JY, White DJ, Hartenstein V, Eliceiri K, Tomancak P, Cardona A (2012) Fiji: an open-source platform for biological-image analysis. Nat Methods 9:676-682.

Schiweck J, Eickholt BJ, Murk K (2018) Important shapeshifter: mechanisms allowing astrocytes to respond to the changing nervous system during development, injury and disease. Front Cell Neurosci 12:261.

Seidl AH (2014) Regulation of conduction time along axons. Neuroscience 276:126-134

Simard AR, Rivest S (2004) Bone marrow stem cells have the ability to populate the entire central nervous system into fully differentiated parenchymal microglia. FASEB J 18:998-1000.

Sinclair JL, Fischl MJ, Alexandrova O, $\mathrm{He} \beta \mathrm{M}$, Grothe B, Leibold C, Kopp-Scheinpflug C (2017) Sound-evoked activity influences myelination of brainstem axons in the trapezoid body. $\mathrm{J}$ Neurosci 37:8239-8255

Smith PH, Joris PX, Carney LH, Yin TC (1991) Projections of physiologically characterized globular bushy cell axons from the cochlear nucleus of the cat. J Comp Neurol 304:387-407.

Spangenberg EE, Lee RJ, Najafi AR, Rice RA, Elmore MR, BlurtonJones M, West BL, Green KN (2016) Eliminating microglia in Alzheimer's mice prevents neuronal loss without modulating amyloid-beta pathology. Brain 139:1265-1281.

Stanley ER, Berg KL, Einstein DB, Lee PS, Pixley FJ, Wang Y, Yeung YG (1997) Biology and action of colony-stimulating factor-1. Mol Reprod Dev 46:4-10.

Taschenberger H, Leao RM, Rowland KC, Spirou GA, Von Gersdorff H (2002) Optimizing synaptic architecture and efficiency for highfrequency transmission. Neuron 36:1127-1143.

Tay TL, Mai D, Dautzenberg J, Fernández-Klett F, Lin G, Sagar Datta M, Drougard A, Stempfl T, Ardura-Fabregat A, Staszewski O, Margineanu A, Sporbert A, Steinmetz LM, Pospisilik JA, Jung S, Priller J, Grün D, Ronneberger O, Prinz M (2017) A new fate mapping system reveals context-dependent random or clonal expansion of microglia. Nat Neurosci 20:793-803.

Tollin DJ (2003) The lateral superior olive: a functional role in sound source localization. Neuroscientist 9:127-143.

Tremblay ME, Lowery RL, Majewska AK (2010) Microglial interactions with synapses are modulated by visual experience. PLoS Biol 8:e1000527.

Trussell LO (1999) Synaptic mechanisms for coding timing in auditory neurons. Annu Rev Physiol 61:477-496.

Vainchtein ID, Molofsky AV (2020) Astrocytes and microglia: in sickness and in health. Trends Neurosci 43:144-154.

Vainchtein ID, Chin G, Cho FS, Kelley KW, Miller JG, Chien EC, Liddelow SA, Nguyen PT, Nakao-Inoue H, Dorman LC, Akil O, Joshita S, Barres BA, Paz JT, Molofsky AB, Molofsky AV (2018) Astrocyte-derived interleukin-33 promotes microglial synapse engulfment and neural circuit development. Science 359:1269-1273.

Wang T, Van Woerden GM, Elgersma Y, Borst JGG (2018) Enhanced transmission at the calyx of Held synapse in a mouse model for Angelman syndrome. Front Cell Neurosci 11:418.

Weinhard L, Di Bartolomei G, Bolasco G, Machado P, Schieber NL, Neniskyte U, Exiga M, Vadisiute A, Raggioli A, Schertel A, Schwab Y, Gross CT (2018) Microglia remodel synapses by presynaptic trogocytosis and spine head filopodia induction. Nat Commun 9:1228.

Whitelaw BS (2018) Microglia-mediated synaptic elimination in neuronal development and disease. J Neurophysiol 119:1-4.

Wieghofer P, Knobeloch KP, Prinz M (2015) Genetic targeting of microglia. Glia 63:1-22.

Willis EF, Macdonald KPA, Nguyen QH, Garrido AL, Gillespie ER, Harley SBR, Bartlett PF, Schroder WA, Yates AG, Anthony DC, Rose-John S, Ruitenberg MJ, Vukovic J (2020) Repopulating microglia promote brain repair in an IL-6-dependent manner. Cell 180:833-846.e16.

Wofchuk ST, Rodnight R (1995) Age-dependent changes in the regulation by external calcium ions of the phosphorylation of glial fibrillary acidic protein in slices of rat hippocampus. Brain Res Dev Brain Res 85:181-186.

Wolf SA, Boddeke HW, Kettenmann H (2017) Microglia in physiology and disease. Annu Rev Physiol 79:619-643.

Zhang Y, Zhao L, Wang X, Ma W, Lazere A, Qian Hh, Zhang J, AbuAsab M, Fariss RN, Roger JE, Wong WT (2018) Repopulating retinal microglia restore endogenous organization and function under CX3CL1-CX3CR1 regulation. Sci Adv 4:eaap8492. 